\title{
What Is the Consumption-CAPM Missing? An Information-Theoretic Framework for the Analysis of Asset Pricing Models,
}

\author{
Anisha Ghosh \\ Tepper School of Business, Carnegie Mellon University \\ Christian Julliard \\ London School of Economics and CEPR
}

Alex P. Taylor

Alliance Manchester Business School

\begin{abstract}
We consider asset pricing models in which the SDF can be factorized into an observable component and a potentially unobservable one. Using a relative entropy minimization approach, we nonparametrically estimate the SDF and its components. Empirically, we find the SDF has a business-cycle pattern and significant correlations with market crashes and the Fama-French factors. Moreover, we derive novel bounds for the SDF that are tighter and have higher information content than existing ones. We show that commonly used consumption-based SDFs correlate poorly with the estimated one, require high risk aversion to satisfy the bounds and understate market crash risk. (JEL G11, G12, G13, C52)
\end{abstract}

Received December 19, 2012; editorial decision January 17, 2016 by Editor Pietro Veronesi.

The absence of arbitrage opportunities implies the existence of a pricing kernel, also known as the stochastic discount factor (SDF), such that the equilibrium price of a traded security can be represented as the conditional expectation of the future payoff discounted by the pricing kernel. The standard consumption-based asset pricing model, within the representative agent and time-separable power utility framework, identifies the pricing kernel as a simple parametric function of consumption growth. However, pricing kernels based

\footnotetext{
We benefited from helpful comments from Mike Chernov, George Constantinides, Darrell Duffie, Bernard Dumas, Burton Hollifield, Ravi Jagannathan, Nobu Kiyotaki, Albert Marcet, Bryan Routledge, and seminar and conference participants at Carnegie Mellon University, the London School of Economics, INSEAD, Johns Hopkins University, 2011 Adam Smith Asset Pricing Conference, 2011 NBER Summer Institute, 2011 Society for Financial Econometrics Conference, 2011 CEPR ESSFM at Gerzensee, 2012 Annual Meeting of the American Finance Association. We are extremely thankful for thoughtful and stimulating inputs from Pietro Veronesi (the editor) and an anonymous referee. Christian Julliard thanks the Economic and Social Research Council (UK) [ES/K002309/1] for financial support. Send correspondence to Christian Julliard, Department of Finance, London School of Economics, London, WC2A2AE, U.K.; telephone: +44 (0) 207107 5366. E-mail: c.julliard@ lse.ac.uk.

(C) The Author 2016. Published by Oxford University Press on behalf of The Society for Financial Studies. This is an Open Access article distributed under the terms of the Creative Commons Attribution License (http://creativecommons.org/licenses/by/4.0/), which permits unrestricted reuse, distribution, and reproduction in any medium, provided the original work is properly cited. doi:10.1093/rfs/hhw075 
on consumption growth alone cannot explain either the historically observed levels of returns, giving rise to the equity premium and risk-free rate puzzles (e.g., Mehra and Prescott 1985; Weil 1989), or the cross-sectional dispersion of returns between different classes of financial assets (e.g., Hansen and Singleton 1983; Mankiw and Shapiro 1986; Breeden et al, 1989; Campbell 1996) 11

Nevertheless, considerable empirical evidence suggests that consumption risk does matter for explaining asset returns (e.g., Lettau and Ludvigson 2001a, 2001b; Parker and Julliard 2005; Hansen et al. 2008; Savov 2011). Therefore, a burgeoning literature has developed based on modifying the preferences of investors and/or the structure of the economy. In such models the resultant pricing kernel can be factorized into an observable component consisting of a parametric function of consumption growth, and a potentially unobservable, model-specific component. Prominent examples in this class include the external habit model in which the additional component consists of a function of the habit level Campbell and Cochrane 1999; Menzly et al.2004), the long run risks model based on recursive preferences in which the additional component consists of the return on total wealth Bansal and Yaron 2004, and models with housing risk in which the additional component consists of the growth in the expenditure share on nonhousing consumption Piazzesi et al. 2007). The additional and potentially unobserved component may also capture deviations from rational expectations (e.g., Brunnermeier and Julliard 2007), models with robust control (e.g., Hansen and Sargent 2010), heterogeneous agents (e.g., Constantinides and Duffie 1996), ambiguity aversion (e.g., Ulrich 2010), and a liquidity factor arising from solvency constraints (e.g.. Lustig and Nieuwerburgh 2005).

In this paper, we propose a new methodology to analyze dynamic asset pricing models, such as those described above, for which the SDF can be factorized into an observable component and a potentially unobservable one. Our no-arbitrage approach allows us to (1) nonparametrically estimate, from the data, the time series of the unobserved pricing kernel under a set of asset pricing restrictions, (2) construct entropy bounds to assess the empirical plausibility of candidate SDFs, and (3) estimate, given a fully observable pricing kernel, the minimum (in the information sense) adjustment of the SDF needed to correctly price asset returns. This methodology provides useful diagnostics tools for studying the ways in which various models might fail empirically, and allows us to characterize some properties that a successful model must satisfy.

First, we show that, given a set of asset returns and consumption data, a relative entropy minimization approach can be used to nonparametrically extract the time series of both the SDF and the unobservable component of

1 Recently, Julliard and Ghosh 2012 show that pricing kernels based on consumption growth alone cannot explain either the equity premium puzzle, or the cross-section of asset returns, even after taking into account the possibility of rare disasters. 
the SDF (if any). This method is equivalent to maximising the expected riskneutral likelihood under a set of no arbitrage restrictions. Moreover, given a fully observable pricing kernel, this procedure identifies the minimum amount of extra information that needs to be added to the SDF to enable it to price asset returns correctly. Along this dimension, our paper is close in spirit to the long tradition of using asset (mostly options) prices to estimate the risk-neutral probability measure (see, e.g. Jackwerth and Rubinstein 1996;:Ait-Sahalia and Lo 1998) and use this information to extract an implied pricing kernel (see, e.g., Ait-Sahalia and Lo 2000; Rosenberg and Engle 2002; Ross 2011).

Empirically, our estimated time series for the unobservable pricing kernel is substantially (but far from perfectly) correlated with the Fama and French 1993) factors, for a variety of sample frequencies and assets used in the estimation (even using only assets, like the industry and momentum portfolios, which are not well priced by the Fama-French factors) This suggests that our approach successfully identifies the pricing kernel and provides a rationalization of the empirical success of the Fama and French factors. The estimated SDF has a clear business-cycle pattern but also shows significant and sharp reactions to stock market crashes (even if these crashes do not result in economy-wide contractions). Moreover, we show that, while the SDFs of most of the equilibrium models tend to adequately account for business-cycle risk, they nevertheless fail to show significant reactions to market crashes, and this hampers their ability to price asset returns - that is, all models seem to be missing a market crash risk component.

Second, we construct entropy bounds that restrict the admissible regions for the SDF and the unobservable component of the SDF. Our results complement and improve on the seminal work by Hansen and Jagannathan 1991) that provide minimum variance bounds for the SDF, and Hansen and Jagannathan 1997) (the so-called second Hansen-Jagannathan distance) that identifies the minimum variance (linear) modification of a candidate pricing kernel needed for it to be consistent with asset returns. The use of an entropy metric is also closely related to the works of Stutzen 1995, 1996), who first suggested to construct entropy bounds based on asset pricing restrictions, and Alvarez and Jermann (2005), who derive a lower bound for the volatility of the permanent component of investors' marginal utility of wealth (see also Backus, Chernov, and Zin 2011; Bakshi and Chabi-Yo 2012; Kitamura and Stutzen 2002). We show that a second-order approximation of the risk-neutral entropy bounds ( $Q$-bounds) has the canonical Hansen-Jagannathan bounds as a special case, but are generally tighter since they naturally impose the nonnegativity restriction on the pricing kernel. Using the multiplicative structure of the pricing kernel, we are able to provide novel bounds ( $M$-bounds) that have higher information content, and are tighter, than both the Hansen and Jagannathan 1991) and the

2 This correlation ranges from 0.45 to 0.81 when Fama-French portfolios are used in the estimation of the minimum entropy SDF, but is reduced to a range of $0.43-0.70$ when considering only Industry or Momentum portfolios. 
risk-neutral entropy bounds. Moreover, our approach improves on Alvarez and Jermann (2005) in that a decomposition of the pricing kernel into permanent and transitory components is not required (but is still possible), and we can accommodate an asset space of arbitrary dimension.

Our methodology also can be used to construct bounds ( $\Psi$-bounds) for the potentially unobserved component of the pricing kernel. We show that for models in which the pricing kernel is only a function of observable variables, the $\Psi$-bounds are the tightest ones, and can be satisfied if and only if the model is actually able to correctly price assets. Moreover, when the pricing kernel is fully observable, our $\Psi$-bounds are closely related to the second HansenJagannathan distance: HJ identify the minimum variance linear adjustment, and our approach identifies the minimum entropy multiplicative (or loglinear) adjustment, that would make a candidate pricing kernel consistent with observed asset returns. The key difference between the two approaches is that the entropy one focuses not only on the second moment deviations, but also on all other higher moments. In an empirical example using stock return data, we find that these higher moments play an important role in driving about $22 \%-26 \%$ of the entropy of the estimated pricing kernel.

Third, we demonstrate how our methodology provides useful diagnostic tools to assess the plausibility of some of the most well-known consumption-based asset pricing models, and lends new insights into their empirical performance. For the standard time-separable power utility model, we show that the pricing kernel satisfies the Hansen and Jagannathan 1991) bound for large values of the risk-aversion coefficient and the $Q$ and $M$ bounds for even higher levels of risk aversion. However, the $\Psi$-bound is tighter and is not satisfied for any level of risk aversion. We show that these findings are robust to the use of the long-run consumption risk measure of Parker and Julliard 2005), despite that this measure of consumption risk is able to explain a substantial share of the cross-sectional variation in asset returns with a small risk-aversion coefficient. Considering more general models of dynamic economies, such as models with habit formation, long-run risks in consumption growth, and complementarities in consumption, we find that the SDFs implied by all of them (1) correlate poorly with the filtered SDF, (2) require implausibly high levels of risk-aversion to satisfy the entropy bounds, and (3) tend to understate market crash risk, in particular, the risk associated with market crashes that do not result in recessions. Moreover, the empirical application illustrates that inference based on the entropy bounds delivers results that are much more stable in evaluating the plausibility of a given model across different sets of assets and data frequencies than the cross-sectional $R^{2}$ (that, instead, tends to vary wildly for the same model).

Compared with the previous literature, our nonparametric approach offers five main advantages: (1) it can be used to extract information not only from options, as is common in the literature, but also from any type of financial asset, (2) instead of exclusively relying on the information contained in financial data, 
it allow us to also exploit the information about the pricing kernel contained in the time series of aggregate consumption, thereby connecting our results to macrofinance modeling, (3) the relative entropy extraction of the SDF is akin to a nonparametric maximum likelihood procedure and provides an estimate of its time series, (4) the methodology has considerable generality, and may be applied to any model that delivers well-defined Euler equations and for which the SDF can be factorized into an observable component and an unobservable one (these include investment-based asset pricing models, and models with heterogenous agents, limited stock market participation, and fragile beliefs), (5) it relies not only on the second moment of the pricing kernel but also on all higher moments.

\section{Entropy and the Pricing Kernel}

In the absence of arbitrage opportunities, a strictly positive pricing kernel, $M_{t+1}$, or stochastic discount factor exists, such that the equilibrium price, $P_{i t}$, of any asset $i$ delivering a future payoff, $X_{i t+1}$, is given by

$$
P_{i t}=\mathbb{E}_{t}\left[M_{t+1} X_{i t+1}\right],
$$

where $\mathbb{E}_{t}$ is the rational expectation operator conditional on the information available at time $t$. For a broad class of models, the SDF can be factorized as follows

$$
M_{t}=m(\theta, t) \times \psi_{t},
$$

where $m(\theta, t)$ denotes the time $t$ value of a known, strictly positive, function of observable data and the parameter vector $\theta \in \Theta \subseteq \mathbb{R}^{k}$ with true value $\theta_{0}$, and $\psi_{t}$ is a potentially unobservable component. In the most common case, $m(\theta, t)$ is simply a function of consumption growth, that is, $m(\theta, t)=m\left(\theta, \Delta c_{t}\right)$, where $\Delta c_{t} \equiv \log \frac{C_{t}}{C_{t-1}}$ and $C_{t}$ denotes the time $t$ consumption flow.

Equations (1) and (2) imply that, for any set of tradable assets, the following vector of Euler equations must hold in equilibrium

$$
\mathbf{0}=\mathbb{E}\left[m(\theta, t) \psi_{t} \mathbf{R}_{t}^{e}\right] \equiv \int m(\theta, t) \psi_{t} \mathbf{R}_{t}^{e} d P,
$$

where $\mathbb{E}$ is the unconditional rational expectation operator $3 \mathbf{R}_{t}^{e} \in \mathbb{R}^{N}$ is a vector of excess returns on different tradable assets, and $P$ is the unconditional physical probability measure. Under weak regularity conditions the above pricing restrictions for the SDF can be rewritten as

$$
\mathbf{0}=\int m(\theta, t) \frac{\psi_{t}}{\bar{\psi}} \mathbf{R}_{t}^{e} d P=\int m(\theta, t) \mathbf{R}_{t}^{e} d \Psi \equiv \mathbb{E}^{\Psi}\left[m(\theta, t) \mathbf{R}_{t}^{e}\right],
$$

3 Our setting can accomodate departures from rational expectations as long as the objective and subjective probability measures are absolutely continuos (that is, as long as the two measures have the same zero probability sets). If agents had subjective beliefs of this type, Equation 3 would still hold, with $\mathbb{E}$ denoting rational expectations, but $\psi_{t}$ would contain a change of measure element capturing the discrepancy between subjective beliefs and the rational expectations (see e.g., Hansen 2014). 
where $\bar{x} \equiv \mathbb{E}\left[x_{t}\right]$, and $\frac{\psi_{t}}{\bar{\psi}}=\frac{d \Psi}{d P}$ is the Radon-Nikodym derivative of $\Psi$ with respect to $P$. For the above change of measure to be legitimate, we need absolute continuity of the measures $\Psi$ and $P$.

Therefore, given a set of consumption and asset returns data, for any $\theta$, one can obtain a nonparametric maximum likelihood estimate of the $\Psi$ probability measure as follows:

$$
\Psi^{*}(\theta) \equiv \underset{\Psi}{\arg \min } D(\Psi \| P) \equiv \underset{\Psi}{\arg \min } \int \frac{d \Psi}{d P} \ln \frac{d \Psi}{d P} d P \text { s.t. } \mathbf{0}=\mathbb{E}^{\Psi}\left[m(\theta, t) \mathbf{R}_{t}^{e}\right],
$$

where, for any two absolutely continuous probability measures $A$ and $B, D(A \|$ $B):=\int \ln \frac{d A}{d B} d A \equiv \int \frac{d A}{d B} \ln \frac{d A}{d B} d B$ denotes the relative entropy of $A$ with respect to $B$, that is, the Kullback-Leibler Information Criterion (KLIC) divergence between the measures $A$ and $B$ White 1982). Note that $D(A \| B)$ is always nonnegative, and has a minimum at zero reached when $A$ is identical to $B$. This divergence measures the additional information content of $A$ relative to $B$, and, as pointed out by Robinson 1991, it is very sensitive to any deviation of one probability measure from another. Therefore, the above equation is a relative entropy minimization under the asset pricing restrictions coming from the Euler equations. That is, the minimization in Equation (4) estimates the unknown measure $\Psi$ as the one that adds the minimum amount of additional information needed for the pricing kernel to price assets.

To understand the information-theoretic interpretation of the estimator of $\Psi$, let $F$ be the set of all probability measures on $\mathbb{R}^{N+N^{\prime}}$, where $N^{\prime}$ denotes the dimensionality of the observables in $m(\theta, t)$, and for each parameter vector $\theta \in \Theta$, define the following set of probability measures

$$
\Psi(\theta) \equiv\left\{\psi \in F: \mathbb{E}^{\psi}\left[m(\theta, t) \mathbf{R}_{t}^{e}\right]=\mathbf{0}\right\}
$$

which are also absolutely continuous with respect to the physical measure $P$ in Equation (3). If the observable component of the SDF, $m(\theta, t)$, correctly prices assets at the given value of $\theta$, we have that $P \in \Psi(\theta)$, and $P$ solves Equation (4), delivering a KLIC value of 0 . On the other hand, if $m(\theta, t)$ is not sufficient to price assets, $P$ is not an element of $\Psi(\theta)$, and a positive KLIC distance $D(\Psi \| P)>0$ is attained by the solution $\Psi^{*}(\theta)$. Thus, the estimation approach searches for a $\Psi^{*}(\theta)$ that adds the minimum amount of additional information needed for the pricing kernel to price asset returns.

The above approach also can be used, as first suggested by Stutzen 1995), to recover the risk-neutral probability measure $(Q)$ from the data as

$$
Q^{*} \equiv \underset{Q}{\arg \min } D(Q \| P) \equiv \underset{Q}{\arg \min } \int \frac{d Q}{d P} \ln \frac{d Q}{d P} d P \text { s.t. } \mathbf{0}=\int \mathbf{R}_{t}^{e} d Q \equiv \mathbb{E}^{Q}\left[\mathbf{R}_{t}^{e}\right]
$$

under the restriction that $Q$ and $P$ are absolutely continuous.

The definition of relative entropy, or KLIC, implies that this discrepancy metric is not symmetric; that is, generally $D(A \| B) \neq D(B \| A)$ unless $A$ and 
$B$ are identical (hence their divergence is always zero) 4 This implies that for measuring the information divergence between $\Psi$ and $P$, as well as between $Q$ and $P$, we can also invert the roles of $\Psi$ and $P$ in Equation (4) and the roles of $Q$ and $P$ in Equation (5) to recover $\Psi$ and $Q$ as

$$
\begin{aligned}
\Psi^{*}(\theta) & \equiv \underset{\Psi}{\arg \min } D(P \| \Psi) \equiv \underset{\Psi}{\arg \min } \int \ln \frac{d P}{d \Psi} d P \text { s.t. } \mathbf{0}=\mathbb{E}^{\Psi}\left[m(\theta, t) \mathbf{R}_{t}^{e}\right], \\
Q^{*} & \equiv \underset{Q}{\arg \min } D(P \| Q) \equiv \underset{Q}{\arg \min } \int \ln \frac{d P}{d Q} d P \text { s.t. } \mathbf{0}=\mathbb{E}^{Q}\left[\mathbf{R}_{t}^{e}\right] .
\end{aligned}
$$

The divergence $D(P \| \Psi)$ can be thought of as the information loss from measure $\Psi$ to measure $P$ (and similarly for $D(P \| Q)$ ). This alternative approach, once again, chooses $\Psi$ and $Q$ such that assets are priced correctly and such that the estimated probability measures are as close as possible (that is minimizing the information loss of moving from one measure to the other) to the physical probability measure $P$.

Note that the approaches in Equations (4) and (6) identify $\left\{\psi_{t}\right\}_{t=1}^{T}$ only up to a positive scale constant. Nevertheless, this scaling constant can be recovered from the Euler equation for the risk-free asset (if one is willing to assume that such an asset is observable).

But why should relative entropy minimization be an appropriate criterion for recovering the unknown measures $\Psi$ and $Q$ ? We make this choice for several reasons.

First, as formally shown in Appendix A.1 the KLIC minimizations in Equations (44-77) are equivalent to maximizing the (expected) 5 and $\Psi$ nonparametric likelihood functions in an unbiased procedure for finding the pricing kernel or its $\psi_{t}$ component. Note that this is also the rationale behind the principle of maximum entropy (see, e.g., Jaynes 1957b, 1957a) in physical sciences and Bayesian probability that states that, subject to known testable constraints - the asset pricing Euler restrictions in our case - the probability distribution that best represents our knowledge is the one with maximum entropy or minimum relative entropy in our notation.

Second, the use of relative entropy, due to the presence of the logarithm in the objective functions in Equations [4-(7), naturally imposes the nonnegativity of the pricing kernel. This, for example, is not imposed in the identification of the minimum variance pricing kernel of Hansen and Jagannathan 1991) 6

4 Information theory provides an intuitive way of understanding the asymmetry of the KLIC: $D(A \| B)$ can be thought of as the expected minimum amount of extra information bits necessary to encode samples generated from $A$ when using a code based on $B$ (rather than using a code based on $A$ ). Hence, generally $D(A \| B) \neq D(B \| A)$ since the latter, by the same logic, is the expected information gain necessary to encode a sample generated from $B$ using a code based on $A$.

5 With expectations under the physical measures proxied by their sample analogs

6 Hansen and Jagannathan 1991) offer an alternative bound that imposes this restriction, but it is computationally cumbersome (the minimum variance portfolio is basically an option in this case). See also Hansen, Heaton, and Luttmer 1995). 
Third, our approach to uncover the $\psi_{t}$ component of the pricing kernel satisfies Occam's razor, or the law of parsimony, since it adds the minimum amount of information needed for the pricing kernel to price assets. This is due to the fact that the relative entropy is measured in units of information.

Fourth, it is straightforward to add conditioning information to construct a conditional version of the entropy bounds presented in the next section: given a vector of conditioning variables $\mathbf{Z}_{t-1}$, one simply has to multiply (element by element) the argument of the integral constraints in Equations (4), (5), (6), and (7) by the conditioning variables in $\mathbf{Z}_{t-1}$.

Fifth, there is no ex ante restriction on the number of assets that can be used in constructing $\psi_{t}$, and the approach can naturally handle assets with negative expected rates of return (cf. Alvarez and Jermann 2005.).

Sixth, as implied by the work of Brown and Smith 1990), the use of entropy is desirable if we think that tail events are an important component of the risk measure 7

Finally, this approach is numerically simple when implemented via duality (see, e.g., Csiszan 1975). That is, when implementing the entropy minimization in Equation (4) each element of the series $\left\{\psi_{t}\right\}_{t=1}^{T}$ can be estimated, up to a positive constant scale factor, as

$$
\psi_{t}^{*}(\theta)=\frac{e^{\lambda(\theta)^{\prime} m(\theta, t) \mathbf{R}_{t}^{e}}}{\sum_{t=1}^{T} e^{\lambda(\theta)^{\prime} m(\theta, t) \mathbf{R}_{t}^{e}}}, \quad \forall t,
$$

where $\lambda(\theta) \in \mathbb{R}^{N}$ is the solution to the following unconstrained convex problem

$$
\lambda(\theta) \equiv \arg \min _{\lambda} \frac{1}{T} \sum_{t=1}^{T} e^{\lambda^{\prime} m(\theta, t) \mathbf{R}_{t}^{e}},
$$

and this last expression is the dual formulation of the entropy minimization problem in Equation (4).

Similarly, the entropy minimization in Equation (6) is solved by

$$
\psi_{t}^{*}(\theta)=\frac{1}{T\left(1+\lambda(\theta)^{\prime} m(\theta, t) \mathbf{R}_{t}^{e}\right)}, \quad \forall t,
$$

where $\lambda(\theta) \in \mathbb{R}^{N}$ is the solution to

$$
\lambda(\theta) \equiv \arg \min _{\lambda}-\sum_{t=1}^{T} \log \left(1+\lambda^{\prime} m(\theta, t) \mathbf{R}_{t}^{e}\right),
$$

and this last expression is the dual formulation of the entropy minimization problem in Equation (6).

7 Brown and Smith 1990 develop what they call "a Weak Law of Large Numbers for rare events;" that is, they show that the empirical distribution observed in a very large sample converges to the distribution that minimizes the relative entropy. 
Note also that the above duality results imply that the number of free parameters available in estimating $\{\psi\}_{t=1}^{T}$ is equal to the dimension of (the Lagrange multiplier) $\lambda$; that is, it is simply equal to the number of assets considered in the Euler equation.

Moreover, since the $\lambda(\theta)$ 's in Equations (9) and 110 are akin to extremum estimators (see, e.g., Havashil2000, Ch. 7), under standard regularity conditions (see, e.g., Amemiya 1985, Theorem 4.1.3), one can construct asymptotic confidence intervals for both $\left\{\psi_{t}\right\}_{t=1}^{T}$ and the entropy bounds presented in the next section.

To summarize, we estimate the $\psi_{t}$ component of the SDF nonparametrically, using the relative entropy-minimizing procedures in Equations (4) and (6). The estimate $\left\{\psi_{t}^{*}(\theta)\right\}_{t=1}^{T}$ is then multiplied with the observable component $m(\theta, t)$ to obtain the overall SDF, $M_{t}^{*}=m(\theta, t) \psi_{t}^{*}(\theta)$. Since we have proposed two different relative entropy minimization approaches, we obtain two different estimates of the SDF given the data. Asymptotically, the two should be identical given the MLE property of these procedures. Nevertheless, in any finite sample they potentially could be very different. As shown in our empirical analysis, the two estimates are very close to each other, suggesting that their asymptotic behavior is well approximated in our sample.

\subsection{Entropy bounds}

Based on the relative entropy estimation of the pricing kernel and the component $\psi$ outlined in the previous section, we now turn our attention to the derivation of a set of entropy bounds for the SDF, $M$, and the components of the SDF.

Dynamic equilibrium asset pricing models identify the SDF as a parametric function of variables determined by the consumers' preferences and the state variables driving the economy. Substantial research efforts have been devoted to developing diagnostic methods to assess the empirical plausibility of candidate SDFs, as well as to provide guidance for the construction and testing of other - more realistic - asset pricing theories.

The seminal work by Hansen and Jagannathan 1991) identifies, in a modelfree no-arbitrage setting, a variance-minimizing benchmark $\mathrm{SDF}, M_{t}^{*}(\bar{M})$, whose variance places a lower bound on the variances of other admissible SDFs:

Definition 1 (Canonical $H J$-bound). For each $E\left[M_{t}\right]=\bar{M}$, the Hansen and Jagannathan 1991) minimum variance SDF is

$$
M_{t}^{*}(\bar{M}) \equiv \underset{\left\{M_{t}(\bar{M})\right\}_{t=1}^{T}}{\operatorname{argmin}} \sqrt{\operatorname{Var}\left(M_{t}(\bar{M})\right)} \text { s.t. } \mathbf{0}=\mathbb{E}\left[\mathbf{R}_{t}^{e} M_{t}(\bar{M})\right] .
$$

The solution to the above minimization is $M_{t}^{*}(\bar{M})=\bar{M}+\left(\mathbf{R}_{t}^{e}-\mathbb{E}\left[\mathbf{R}_{t}^{e}\right]\right)^{\prime} \beta_{\bar{M}}$, where $\beta_{\bar{M}}=\operatorname{Cov}\left(\mathbf{R}_{t}^{e}\right)^{-1}\left(-\bar{M} \mathbb{E}\left[\mathbf{R}_{t}^{e}\right]\right)$, and any candidate stochastic discount factor $M_{t}$ must satisfy $\operatorname{Var}\left(M_{t}(\bar{M})\right) \geq \operatorname{Var}\left(M_{t}^{*}(\bar{M})\right)$. 
The $H J$-bound offers a natural benchmark for evaluating the potential of an equilibrium asset pricing model since, by construction, any SDF consistent with observed data should have a variance not smaller than that of $M_{t}^{*}(\bar{M})$. However, the identified minimum variance SDF does not impose the nonnegativity constraint on the pricing kernel. In fact, since $M_{t}^{*}(\bar{M})$ is a linear function of returns, the restrction is not generally natisfied 8

As noticed in Stutzen 1995), using the Kullback-Leibler Information Criterion minimization in Equation (5), one can construct an entropy bound for the risk-neutral probability measure that naturally imposes the nonnegativity constraint on the pricing kernel. We generalize the idea of using an entropy minimization approach to construct risk-neutral bounds - $Q$-bounds - for the pricing kernel. For a given risk-neutral probability measure $Q$ with RadonNikodym derivative $\frac{d Q}{d P}=\frac{M_{t}}{\bar{M}}$, we use $D(P \| Q)$ and $D\left(P \| \frac{M_{t}}{\bar{M}}\right)$ interchangeably, that is, $D\left(P \| \frac{M_{t}}{\bar{M}}\right) \equiv D(P \| Q) \equiv \int \ln \left(\frac{d P}{d Q}\right) d P \equiv-\int \ln \left(\frac{M_{t}}{\bar{M}}\right) d P$. Similarly, $D\left(\frac{M_{t}}{\bar{M}} \|\right.$ $P) \equiv D(Q \| P) \equiv \int \ln \left(\frac{d Q}{d P}\right) d Q \equiv \int \frac{d Q}{d P} \ln \left(\frac{d Q}{d P}\right) d P \equiv \int \frac{M_{t}}{\bar{M}} \ln \left(\frac{M_{t}}{\bar{M}}\right) d P$.

Definition 2 (Q-bounds). We define the following risk-neutral probability bounds for any candidate stochastic discount factor $M_{t}$ :

1. Q1-bound:

$$
D\left(P \| \frac{M_{t}}{\bar{M}}\right) \equiv \int-\ln \frac{M_{t}}{\bar{M}} d P \geqslant D\left(P \| Q^{*}\right),
$$

where $Q^{*}$ solves Equation (7).

2. Q2-bound Stutzen 1995):

$$
D\left(\frac{M_{t}}{\bar{M}} \| P\right) \equiv \int \frac{M_{t}}{\bar{M}} \ln \frac{M_{t}}{\bar{M}} d P \geqslant D\left(Q^{*} \| P\right),
$$

where $Q^{*}$ solves Equation (5).

These bounds, like the $H J$-bound, use only the information contained in asset returns, but, differently from the latter, they impose the restriction that the pricing kernel must be positive. Moreover, under mild regularity conditions, we show that (see Remark2 in Appendix A.2, to a second-order approximation, the problem of constructing canonical $H J$-bounds and $Q$-bounds are equivalent, in the sense that approximated $Q$-bounds identify the minimum variance bound for the SDF 9 The intuition behind this result is simple: (1) a second-order

8 We call the bound in Definition 1 the "canonical" $H J$-bound since Hansen and Jagannathan 1991, 1997) also provide an alternative bound, that imposes the non-negativity of the pricing kernel, but that is computationally more complex.

9 The (sufficient, but not necessary) regularity conditions required for the approximation result are typically satisfied in consumption-based asset pricing models. 
approximation of (the $\log$ of) a smooth pdf delivers an approximately Gaussian distribution (see, e.g., Schervish 1995), (2) the relative entropy of a Gaussian distribution is proportional to its variance, and (3) the diffusion invariance principle (see, e.g., Duffie 2005, Appendix D) implies that in the continuous time limit the (equivalent) change of measure does not change the volatility.

Both the $H J$ and $Q$ bounds described above use only information about asset returns, but not information about consumption growth or the structure of the pricing kernel. Instead, we propose a novel approach that, while also imposing the nonnegativity of the pricing kernel, (1) takes into account more information about the form of the pricing kernel, therefore delivering sharper bounds, and (2) allows us to construct information bounds for both the pricing kernel as a whole and for its individual components.

Consider an SDF that, as in Equation (2), can be factorized into two components, that is, $M_{t}=m(\theta, t) \times \psi_{t}$, where $m(\theta, t)$ is a known nonnegative function of observable variables (generally consumption growth) and the parameter vector $\theta$, and $\psi_{t}$ is a potentially unobservable component. A large class of equilibrium asset pricing models, including ones with time-separable power utility with a constant coefficient of relative-risk aversion, external habit formation, recursive preferences, durable consumption goods, housing, and disappointment aversion fall into this framework. Based on the above factorization of the SDF, we can define the following bounds.

Definition 3 ( $M$-bounds). For any candidate stochastic discount factor of the form in Equation (2), and given any choice of the parameter vector $\theta$, we define the following bounds:

1. M1-bound:

$$
\begin{aligned}
D\left(P \| \frac{M_{t}}{\bar{M}}\right) \equiv \int-\ln \frac{M_{t}}{\bar{M}} d P & \geqslant D\left(P \| \frac{m(\theta, t) \psi_{t}^{*}}{\overline{m(\theta, t) \psi_{t}^{*}}}\right) \\
& \equiv \int-\ln \frac{m(\theta, t) \psi_{t}^{*}}{\overline{m(\theta, t) \psi_{t}^{*}}} d P
\end{aligned}
$$

where $\psi_{t}^{*}$ solves Equation (6) and $\overline{m(\theta, t) \psi_{t}^{*}} \equiv \mathbb{E}\left[m(\theta, t) \psi_{t}^{*}\right]$.

2. M2-bound:

$$
\begin{aligned}
D\left(\frac{M_{t}}{\bar{M}} \| P\right) \equiv \int \frac{M_{t}}{\bar{M}} \ln \frac{M_{t}}{\bar{M}} d P & \geqslant D\left(\frac{m(\theta, t) \psi_{t}^{*}}{\overline{m(\theta, t) \psi_{t}^{*}}} \| P\right) \\
& \equiv \int \frac{m(\theta, t) \psi_{t}^{*}}{\overline{m(\theta, t) \psi_{t}^{*}}} \ln \frac{m(\theta, t) \psi_{t}^{*}}{\overline{m(\theta, t) \psi_{t}^{*}}} d P,
\end{aligned}
$$

where $\psi_{t}^{*}$ solves Equation 4 . 
The above bounds for the SDF are tighter than the $Q$-bounds since we have that (the minimum entropy risk-neutral probability measure is denoted $Q^{*}$ )

$$
D\left(P \| \frac{m(\theta, t) \psi_{t}^{*}}{\overline{m(\theta, t) \psi_{t}^{*}}}\right) \geq D\left(P \| Q^{*}\right) \text { and } D\left(\frac{m(\theta, t) \psi_{t}^{*}}{\overline{m(\theta, t) \psi_{t}^{*}}} \| P\right) \geq D\left(Q^{*} \| P\right),
$$

by construction, and are also more informative since not only is the information contained in asset returns used in their construction but also (1) the structure of the pricing kernel in Equation (2) and (2) the information contained in $m(\theta, t)$.

Information about the SDF also can be elicited by constructing bounds for the $\psi_{t}$ component itself. Given the $m(\theta, t)$ component, these bounds identify the minimum amount of information that $\psi_{t}$ should add for the pricing kernel $M_{t}$ to be able to price asset returns 10

Definition 4 ( $\Psi$-bounds). For any candidate stochastic discount factor of the form in Equation [2], and given any choice of the parameter vector $\theta$, two lower bounds for the relative entropy of $\psi_{t}$ are defined as

1. $\Psi$ 1-bound:

$$
D\left(P \| \frac{\psi_{t}}{\bar{\psi}}\right) \equiv-\int \ln \frac{\psi_{t}}{\bar{\psi}} d P \geqslant D\left(P \| \frac{\psi_{t}^{*}}{\bar{\psi}^{*}}\right)
$$

where $\psi_{t}^{*}$ solves Equation 6 ;

2. $\Psi$ 2-bound

$$
D\left(\frac{\psi_{t}}{\bar{\psi}} \| P\right) \equiv \int \frac{\psi_{t}}{\bar{\psi}} \ln \frac{\psi_{t}}{\bar{\psi}} d P \geqslant D\left(\frac{\psi_{t}^{*}}{\bar{\psi}^{*}} \| P\right),
$$

where $\psi_{t}^{*}$ solves Equation (4).

Besides providing an additional check for any candidate SDF, the $\Psi$-bounds are useful in that a simple comparison of $D\left(\frac{\psi_{t}^{*}}{\bar{\psi}^{*}} \| P\right), D\left(\frac{m(\theta, t)}{\overline{m(\theta, t)}} \| P\right)$, and $D\left(Q^{*} \| P\right)$ can provide a very informative decomposition in terms of the entropy contribution to the pricing kernel logically similar to the widely used variance decomposition analysis. For example, if $D\left(\frac{\psi_{t}^{*}}{\bar{\psi}^{*}} \| P\right)$ happens to be close to $D\left(Q^{*} \| P\right)$, while $D\left(\frac{m(\theta, t)}{m(\theta, t)} \| P\right)$ is substantially smaller, the decomposition implies that most of the ability of the candidate SDF to price assets comes from the $\psi_{t}$ component.

Note also that, in principle, a volatility bound, similar to the Hansen and Jagannathan 1991) bound for the pricing kernel, can be constructed for the $\psi_{t}$

10 As for the $Q$ and $M$ bounds, we use $D(P \| \Psi)$ and $D\left(P \| \frac{\psi_{t}}{\bar{\psi}}\right)$, as well as $D(\Psi \| P)$ and $D\left(\frac{\psi_{t}}{\bar{\psi}} \| P\right)$, interchangeably. 
component. Such a bound, presented in Definition 5 of Appendix $\$$.2 identifies a minimum variance $\psi_{t}^{*}\left(\bar{\psi}^{*}\right)$ component with standard deviation given by

$$
\sigma_{\psi^{*}}=\bar{\psi}^{*} \sqrt{\mathbb{E}\left[\mathbf{R}_{t}^{e} m(\theta, t)\right]^{\prime} \operatorname{Var}\left(\mathbf{R}_{t}^{e} m(\theta, t)\right)^{-1} \mathbb{E}\left[\mathbf{R}_{t}^{e} m(\theta, t)\right] .}
$$

This bound, as the entropy-based $\Psi$-bounds in Definition 4 uses information about the structure of the SDF but, differently from the latter, does not constrain $\psi_{t}$ and $M_{t}$ to be nonnegative as implied by economic theory. Moreover, using the same approach employed in Remark 2 this last bound can be obtained as a second-order approximation of the entropy-based $\Psi$-bounds in Definition4

Equation [14], viewed as a second-order approximation to the entropy $\Psi$ bounds, also makes clear why bounds based on the decomposition of the pricing kernel as $M_{t}=m(\theta, t) \psi_{t}$ offer sharper inference than do bounds based on only $M_{t}$. Consider, for example, the case in which the candidate SDF takes the form $M_{t}=m(\theta, t)$, that is, $\psi_{t}=1$ for any $t$. In this case, a $\tilde{\theta}$ can easily exist such that

$$
\operatorname{Var}\left(M_{t}(\tilde{\theta})\right) \equiv \operatorname{Var}(m(\tilde{\theta}, t)) \geq \operatorname{Var}\left(M_{t}^{*}(\bar{M})\right),
$$

where $\operatorname{Var}\left(M_{t}^{*}(\bar{M})\right)$ is the Hansen and Jagannathan 1991) bound in Definition 1, that is, a $\tilde{\theta}$ exists such that the $H J$-bound is satisfied. Nevertheless, the existence of such $\tilde{\theta}$ does not imply that the candidate SDF is able to price asset returns. This would be the case if and only if the volatility bound for $\psi_{t}$ is also satisfied since, from Equation [14], we have that under the assumption of constant $\psi_{t}$ the bound can be satisfied only if $\mathbb{E}\left[\mathbf{R}_{t}^{e} m\left(\theta_{0}, t\right)\right] \equiv \mathbb{E}\left[\mathbf{R}_{t}^{e} M_{t}\left(\theta_{0}\right)\right]=\mathbf{0}$, that is, only if the candidate SDF is able to price asset returns.

1.1.1 Residual $\psi$ and the second Hansen-Jagannathan distance. If we want to evaluate a model of the form $M_{t}=m(\theta, t)$ - that is, a model without an unobservable component - the $\Psi$-bounds will offer a tight selection criterion since, under the null of the model being true, we should have $D\left(\frac{\psi_{t}^{*}}{\bar{\psi}^{*}} \| P\right)=$ $D\left(P \| \frac{\psi_{t}^{*}}{\bar{\psi}^{*}}\right)=0$, and this is a tighter bound than the $H J, Q$, and $M$ bounds defined above. The intuition for this is simple: $Q$-bounds (and $H J$-bounds) require the model under test to deliver at least as much relative entropy (variance) as the minimum relative entropy (variance) SDF, but they do not require that the $m(\theta, t)$ under scrutiny also should be able to price the assets. That is, it might be the case - as in practice we will show is the case - that for some values of $\theta$ both the $Q$-bounds and the $H J$-bounds will be satisfied, but nevertheless the SDF grossly violates the pricing restrictions in the Euler Equation (37.

Note that when considering a model of the form $M_{t}=m(\theta, t)$, the estimated $\psi^{*}$ component is a residual one - that is, it captures what is missed, for pricing assets correctly, by the pricing kernel under scrutiny. The residual $\psi^{*}$ and the entropy bounds are also closely related to the second Hansen and Jagannathan bound. Given a model that identifies a SDF $M$, Hansen and Jagannathan 1997) assume that portfolio payoffs are elements of an Hilbert space and consider the 
minimum squared deviation between $M$ and a pricing kernel $q \in \mathcal{M}$ (or $\mathcal{M}^{+}$if nonnegativity is imposed), where $\mathcal{M}$ denotes the set of all admissible SDFs. That is, the second $\mathrm{HJ}$ distance is defined as

$$
d_{H J}^{2}:=\min _{q \in \mathcal{M}} \mathbb{E}\left[\left(M_{t}-q_{t}\right)^{2}\right] .
$$

Note that $q \in \mathcal{M}$ can be rewritten as $q \in L^{2}$, which satisfies the pricing restriction (1), that is,

$$
d_{H J}^{2} \equiv \min _{q \in L^{2}} \mathbb{E}\left[\left(M_{t}-q_{t}\right)^{2}\right] \text { s.t. } \mathbf{0}=\mathbb{E}\left[q_{t} \mathbf{R}_{t}^{e}\right] \equiv \mathbb{E}^{Q}\left[\mathbf{R}_{t}^{e}\right] .
$$

Note that the constraint in the above formulation is the same one that we impose for constructing our entropy bounds.

In practice, the second HJ bound looks for the minimum - in a least squares sense - linear adjustment that makes $M_{t}-\lambda^{\prime} \mathbf{R}_{t}^{e}$ an admissible SDF (where $\lambda$ arises from the linear projection of $M$ on the space of returns). This idea of minimum adjustment of the second $\mathrm{HJ}$ distance is strongly connected to our $M$ and $\Psi$ bounds and residual $\psi$.

Consider the decomposition $M_{t}=m(\theta, t) \psi_{t}$ in its extreme form: $M_{t} \equiv m(\theta, t)$; that is, the case in which the candidate SDF is fully observable and, under the null of the model under scrutiny, $\psi^{m}$ (the model-implied $\psi$ ) should simply be a constant. In this case, we can estimate a residual $\left\{\psi_{t}^{*}\right\}_{t=1}^{T}$ that should be constant if the model is correct. In this case, the $M 1$-bound defines the distance

$$
d_{M 1}=\min _{\left\{\psi_{t}\right\}_{t=1}^{T}} D\left(P \| M_{t} \psi_{t}\right)-D\left(P \| M_{t}\right) \equiv \min _{\left\{\psi_{t}\right\}_{t=1}^{T}} D\left(P \| \psi_{t}\right) \text { s.t. } \mathbf{0}=\mathbb{E}\left[q_{t} \mathbf{R}_{t}^{e}\right],
$$

where $q_{t}:=M_{t} \psi_{t}$, and we have normalized $\psi_{t}$ to have unit mean to simplify exposition, and note that the second equality is nothing but the $\Psi 1$ bound. Note that in this case we have $\log \psi_{t} \equiv \log q_{t}-\log M_{t}$. That is, while the second HJ distance focuses on the deviation between $q$ and $M$, our entropy approach focuses on the $\log$ deviations. By construction, $M_{t} \psi_{t}^{*} \in \mathcal{M}$ (or $\mathcal{M}^{+}$if $M$ is nonnegative); that is, once again the relative entropy minimization identifies an admissible SDF in the Hansen and Jagannathan 1997) sense. To illustrate the link between the second $\mathrm{HJ}$ distance and the $d_{M 1}$ distance above, we follow the cumulant expansion approach of Backus, Chernov, and Zin 2014). Recall that the cumulant-generating function (that is, the log of the moment-generating function) of a random variable $\ln x_{t}$ is

$$
k^{x}(s)=\ln \mathbb{E}\left[e^{s \ln x_{t}}\right],
$$

and, with appropriate regularity conditions, it admits the power series expansion

$$
k^{x}(s)=\sum_{j=1}^{\infty} \kappa_{j}^{x} \frac{s^{j}}{j !},
$$

where the $j$-th cumulant, $\kappa_{j}$, is the $j$-th derivative of $k^{x}(s)$ evaluated at $s=0$. That is, $\kappa_{j}^{x}$ captures the $j$-th moment of the variable $\ln x_{t}$; that is, $\kappa_{1}^{x}$ reflects 
the mean of the variable, $\kappa_{2}^{x}$ the variance, $\kappa_{3}^{x}$ the skewness, $\kappa_{4}^{x}$ the kurtosis, and so on 11

Using the cumulant expansion, the $d_{M 1}$ distance above can be rewritten as

$$
d_{M 1}=\frac{\kappa_{2}^{\psi^{*}}}{2 !}+\frac{\kappa_{3}^{\psi^{*}}}{3 !}+\frac{\kappa_{4}^{\psi^{*}}}{4 !}+\ldots
$$

where $\kappa_{j}^{\psi^{*}}$ denotes the $j$-th cumulant of $(\log ) \psi^{*}$, and $\psi^{*}$ solves

$$
\underset{\left\{\psi_{t}\right\}_{t=1}^{T}}{\operatorname{argmin}}\left(\frac{\kappa_{2}^{\psi}}{2 !}+\frac{\kappa_{3}^{\psi}}{3 !}+\frac{\kappa_{4}^{\psi}}{4 !}+\ldots\right) \text { s.t. } \mathbf{0}=\mathbb{E}^{\Psi}\left[m(\theta, t) \mathbf{R}_{t}^{e}\right] \text {. }
$$

The above implies that the $\psi^{*}$ component identified by our $M 1$ (and $\Psi 1$ ) bound has a very similar interpretation to the second HJ distance: it provides the minimum - in the entropy sense - multiplicative (or log linear) adjustment that would make $m(\theta, t) \psi_{t}^{*}$ an admissible SDF. The key difference between the second $\mathrm{HJ}$ bound and our $M 1$ bound is that the former only focuses on the minimum second moment deviation, that is, on the variance of $q_{t}-M_{t}$, and our bound takes into consideration not only the second moment (captured by the $\kappa_{2}^{\psi}$ cumulant in Equation (15) but also all other moments (captured by the $\kappa_{j>2}^{\psi}$ cumulants) of the $\log$ deviation $\log q_{t}-\log M_{t} \equiv \log \psi_{t}$. This implies that if skewness, kurtosis, tail probabilities, etc., are relevant for asset pricing, our approach would be more likely to capture these higher moments more effectively than the least squares one. Moreover, note that the cumulantgenerating function cannot be a finite-order polynomial of degree greater than two (see Theorem 7.3.5 of Lukacs 1970). That is, if the mean and variance are not sufficient statistics for the distribution of the true SDF, then all the other higher moments become relevant for characterizing the SDF, and their relevance for asset pricing is captured by our entropy approach given the one-toone mapping between relative entropy and cumulants. In Table A1 of Appendix A.3 we compute the minimum adjustment to the CCAPM SDF required to make it an admissible pricing kernel using both of the above approaches. The results show that, for a wide variety of test assets, the HJD adjustment leads to an SDF that has a close to Gaussian distribution. The relative entropy adjustment, on the other hand, results in an SDF having substantial skewness and kurtosis.

The cumulant decomposition also allows us to assess the relevance of higher moments for pricing asset returns. In particular, with the estimated $\left\{\ln \psi_{t}^{*}\right\}_{t=1}^{T}$ at hand, we can estimate its moments using sample analogs, use these moments to compute the cumulants, and, finally, compute the contribution of the $j$-th cumulant to the total entropy of $\psi^{*}$ as

$$
\frac{\kappa_{j}^{\psi^{*}} / j !}{\sum_{s=2}^{\infty} \kappa_{s}^{\psi^{*}} / s !} \equiv \frac{\kappa_{j}^{\psi^{*}} / j !}{D\left(P \| \Psi^{*}\right)}
$$

11 For instance, if $\ln x_{t} \sim N\left(\mu_{x} ; \sigma_{x}^{2}\right)$, we have $\kappa_{1}^{x}=\mu_{x}, \kappa_{2}^{x}=\sigma_{x}^{2}, \kappa_{j>2}^{x}=0$. 
as well as the total contribution of cumulants of order larger than $j$ as

$$
\frac{\sum_{s=j+1}^{\infty} \kappa_{s}^{\psi^{*}} / s !}{\sum_{s=2}^{\infty} \kappa_{s}^{\psi^{*}} / s !} \equiv \frac{D\left(P \| \Psi^{*}\right)-\sum_{s=2}^{j} \kappa_{s}^{\psi^{*}} / s !}{D\left(P \| \Psi^{*}\right)}
$$

These statistics are important for comparing the informativeness of our bounds relative to the second $\mathrm{HJ}$ distance since, if the minimum variance deviation had all the relevant information for pricing asset returns, we would expect

$$
\frac{D\left(P \| \Psi^{*}\right)-\kappa_{2}^{\psi^{*}} / 2 !}{D\left(P \| \Psi^{*}\right)} \cong 0 \text { and } \frac{\kappa_{j}^{\psi^{*}} / j !}{D\left(P \| \Psi^{*}\right)} \cong 0 \quad \forall j>2 .
$$

As we will show in the empirical section below, this is not the case.

\section{An Illustrative Example: The C-CAPM with Power Utility}

We first illustrate our methodology for the Consumption-CAPM (C-CAPM) of Breeden (1979), Lucas 1978), and Rubinstein 1976), when the utility function is time and state separable with a constant coefficient of relative-risk aversion. For this specification of preferences, the SDF takes the form

$$
M_{t+1}=\delta\left(C_{t+1} / C_{t}\right)^{-\gamma},
$$

where $\delta$ denotes the subjective time discount factor, $\gamma$ is the coefficient of relative-risk aversion, and $C_{t+1} / C_{t}$ denotes the real per capita aggregate consumption growth. Empirically, the above pricing kernel fails to explain (1) the historically observed levels of returns, giving rise to the equity premium and risk-free rate Puzzles (e.g., Mehra and Prescott 1985; Weil 1989), and (2) the cross-sectional dispersion of returns between different classes of financial assets (e.g., Mankiw and Shapiro 1986; Breeden et al. 1989; Campbell 1996; Cochrane 1996).

Parker and Julliard 2005) argue that the covariance between contemporaneous consumption growth and asset returns understates the true consumption risk of the stock market if consumption is slow to respond to return innovations. They propose measuring the risk of an asset by its ultimate risk to consumption, defined as the covariance of its return and consumption growth over the period of the return and many following periods. They show that, while the ultimate consumption risk would correctly measure the risk of an asset if the C-CAPM were true, it may be a better measure of the true risk if consumption responds with a lag to changes in wealth. The ultimate consumption risk model implies the following SDF:

$$
M_{t+1}^{S}=\delta^{1+S}\left(C_{t+1+S} / C_{t}\right)^{-\gamma} R_{t+1, t+1+S}^{f}
$$

where $S$ denotes the number of periods over which the consumption risk is measured and $R_{t+1, t+1+S}^{f}$ is the risk-free rate between periods $t+1$ and $t+1+S$. Note that the standard C-CAPM obtains when $S=0$. Parker and Julliard 2005) 
Panel A

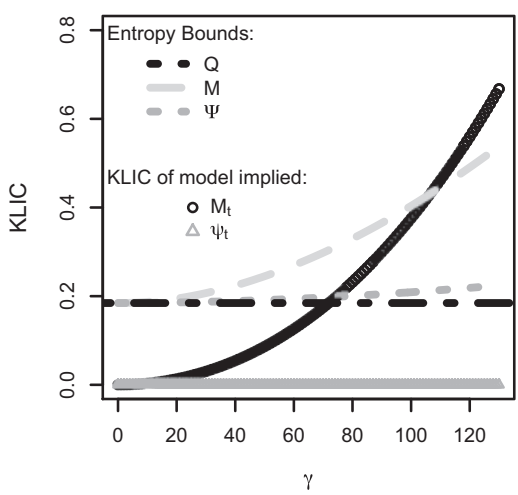

Panel B

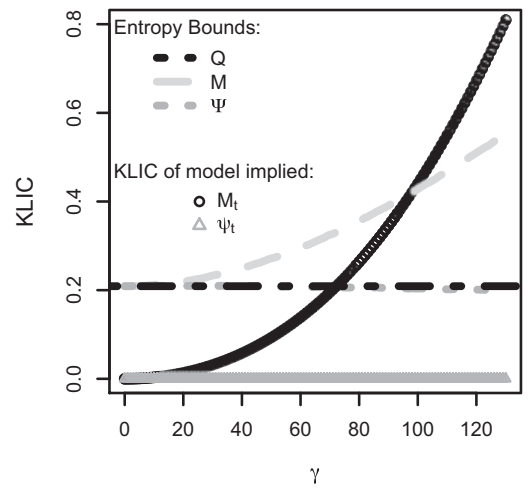

Figure 1

The figure plots the KLIC of the model SDF, $M_{t}=\delta\left(\frac{C_{t}}{C_{t-1}}\right)^{-\gamma}$, and the model $\psi$ (equal to zero in this case), as well as the $Q, M$, and $\Psi$ bounds as a function of the risk-aversion coefficient. The $Q(M)$ bound is satisfied when the KLIC of $M_{t}$ is above it, and the $\Psi$ bound is satisfied when the KLIC of $\psi_{t}$ is above it. Results when $\psi_{t}^{*}$ is estimated using the relative entropy minimization procedures in Equations 6 and 4, respectively, using quarterly data for 1947:Q1-2009:Q4 and the 25 Fama-French portfolios as test assets are shown ( $A$ and $B)$.

show that the specification of the SDF in Equation 20, unlike the one in Equation 19, explains a large fraction of the variation in expected returns across assets for low levels of the risk-aversion coefficient.

The functional forms of the above two SDFs fit into our framework in Equation (2). For the contemporaneous consumption risk model, $\theta=\gamma, m(\theta, t)=$ $\left(C_{t} / C_{t-1}\right)^{-\gamma}$, and $\psi_{t}^{m}=\delta$, a constant, for all $t$. For the ultimate consumption risk model, $\theta=\gamma, m(\theta, t)=\left(C_{t+S} / C_{t-1}\right)^{-\gamma}$, and $\psi_{t}^{m}=\delta^{1+S} R_{t, t+S}^{f}$. Therefore, for each model, we construct entropy bounds for the SDF and the components of the SDF using quarterly data on per capita real personal consumption expenditures on nondurable goods and returns on the 25 Fama-French portfolios over the postwar period 1947:1-2009:4 and compare them with the $H J$ bound $1213 \mathrm{We}$ also obtain the nonparametrically extracted (called "filtered" hereafter) SDF and the components of the SDF for $\gamma=10$. For the ultimate consumption risk model, we set $S=11$ quarters because the fit of the model is the greatest at this value as shown in Parker and Julliard 2005).

Figure 1 panel A, plots the relative entropy (or KLIC) of the filtered and model-implied SDFs and their $\psi$ components as a function of the risk-aversion coefficient $\gamma$ and the $H J, Q 1, M 1$, and $\Psi 1$ bounds for the contemporaneous consumption risk model in Equation 19]. The black curve with circles shows the relative entropy of the model-implied SDF as a function of the risk-aversion

12 See Appendix A.4 for a thorough description of the data.

13 We use the 25 Fama-French portfolios as test assets because they have been used extensively in the literature to test the C-CAPM and also constituted the set of base assets in Parker and Julliard 2005). 
coefficient. For this model, the missing component of the SDF, $\psi_{t}$, is a constant, and hence, it has zero relative entropy for all values of $\gamma$, as shown by the gray straight line with triangles. The gray dashed curve and the gray dotted curve show, respectively, the relative entropy as a function of the risk-aversion coefficient of the filtered SDF and its missing component. The model satisfies the $H J$ bound for very high values of $\gamma \geqslant 64$. It satisfies the $Q 1$ bound for even higher values of $\gamma \geqslant 72$, as shown by the intersection of the horizontal dotted-dashed line and the black curve with circles. The minimum value of $\gamma$, at which the $M 1$ bound is satisfied, is given by the value corresponding to the intersection of the gray dashed curve and the black curve with circle; that is, it is the minimum value of $\gamma$ for which the relative entropy of the model-implied SDF exceeds that of the filtered SDF. The figure shows that this corresponds to $\gamma=107$. Finally, the $\Psi 1$ bound identifies the minimum value of $\gamma$ for which the missing component of the model-implied SDF has a higher relative entropy than the missing component of the filtered SDF. Since the former has zero relative entropy, while the latter has a strictly positive value for all values of $\gamma$, the model fails to satisfy the $\Psi 1$ bound for any value of $\gamma 14$

Panel B shows that very similar results are obtained for the $Q 2, M 2$, and $\Psi 2$ bounds. The $Q 2$ and $M 2$ bounds are satisfied for values of $\gamma$ at least as large as 73 and 99, respectively, while the $\Psi 2$ bound is not satisfied for any value of $\gamma$. Overall, as suggested by the theoretical predictions, the $Q$-bounds are tighter than the $H J$-bound, the $M$-bounds are tighter than the $Q$-bounds, and the $\Psi$-bounds are tighter than the $M$-bounds.

We also construct confidence bands for the above relative entropy bounds using 1,000 bootstrapped samples. The $95 \%$ confidence bands for the $Q 1$ and $Q 2$ bounds extend over the intervals [70.0,109.0] and [69.5, 109.0], respectively, and those for the $M 1$ and $M 2$ bounds cover the intervals [94.5, 157.5] and [86.0,150.0], respectively. Finally, the $\Psi 1$ and $\Psi 2$ bounds are not satisfied for any finite value of the risk-aversion coefficient in any of the bootstrapped samples. The bootstrap results reveal two points. First, it demonstrates the robustness of our approach: the two different definitions of relative entropy produce very similar results. Second, the confidence bands are quite tight in contrast with the large values of the standard error typically obtained when using GMM-type approaches to estimate the risk-aversion parameter.

Figure 2 presents analogous results to Figure1 1 for the ultimate consumption risk model in Equation (20). Panel A shows that the $Q 1$, and $M 1$ bounds

14 Note that Figure 1 plots the relative entropy of the different components of the SDF as functions of the CRRA. The $Q, M$, and $\Psi$ bounds are directly expressed in terms of the risk-aversion coefficient (vertical lines). The $Q$-bound could have been alternatively expressed in terms of entropy, that is, as a horizontal line at $D\left(Q^{*} \| P\right)$ and $D\left(P \| Q^{*}\right)$ in panels $\mathrm{A}$ and $\mathrm{B}$, respectively. One could then have determined what the required minimum CRRA was to satisfy these bounds by computing the minimum CRRA such that the relative entropy of the resulting SDF was at least as large as $D\left(Q^{*} \| P\right)$ or $D\left(P \| Q^{*}\right)$. However, note that the $M$ and $\Psi$ bounds depend on the CRRA and, therefore, cannot be expressed as horizontal lines. We, therefore, choose to represent all the bounds directly in terms of the CRRA (as vertical lines). 
Panel A

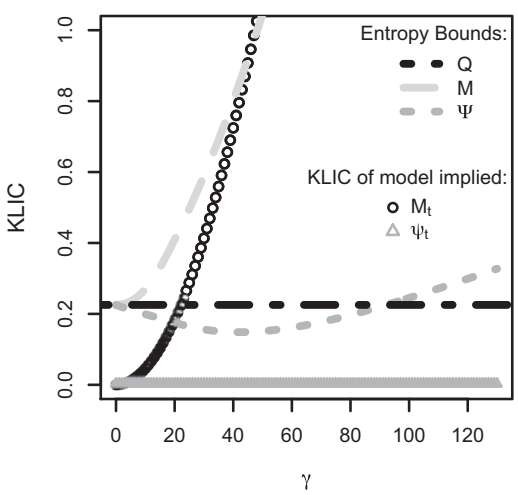

Panel B

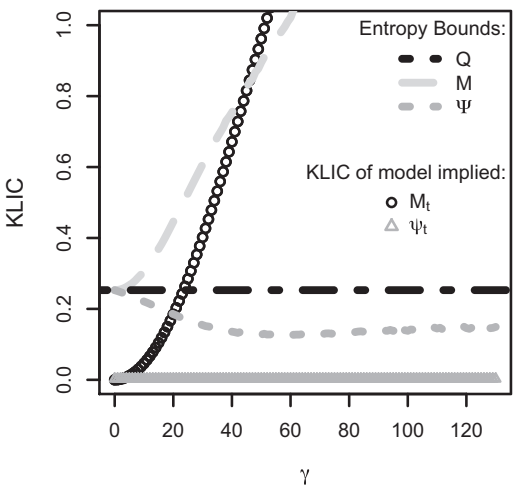

Figure 2

The figure plots the KLIC of the model SDF, $M_{t}=\delta^{1+S}\left(\frac{C_{t+S}}{C_{t-1}}\right)^{-\gamma} R_{t, t+S}^{f}$, and their unobservable components ( $\psi_{t}^{*}$ and the model $\psi$ (equal to zero in this case), as well as the $Q, M$ and $\Psi$ bounds as function of the risk-aversion coefficient. The $Q(M)$ bound is satisfied when the KLIC of $M_{t}$ is above it, and the $\Psi$ bound is satisfied when the KLIC of $\psi_{t}$ is above it. Results when $\psi_{t}^{*}$ is estimated using the relative entropy minimization procedures in Equations 6 and 4, respectively, using quarterly data for 1947:Q1-2009:Q4 and the 25 Fama-French portfolios as test assets are shown $(A$ and $B)$.

are satisfied for $\gamma \geqslant 22,23$, and 46, respectively. These are almost three times, more than three times, and more than two times smaller, respectively, than the corresponding values in Figure 1 panel A, for the contemporaneous consumption risk model. As for the latter model, the $\Psi 1$ bound is not satisfied for any value of $\gamma$. Panel B shows that the $Q 2$ and $M 2$ bounds are satisfied for $\gamma \geqslant 24$ and 47, respectively, while the $\Psi 2$ bound is not satisfied for any value of $\gamma$. The bootstrapped 95\% confidence bands for the $Q 1$ and $Q 2$ bounds extend over the intervals [23.0,35.0] and [24.0,37.0], respectively, and those for the $M 1$ and $M 2$ bounds cover the intervals [36.0,60.0] and [40.0,74.0], respectively. Also, similar to the contemporaneous consumption risk model, the $\Psi 1$ and $\Psi 2$ bounds are not satisfied for any finite value of the risk-aversion coefficient in any of the bootstrapped samples.

It is important to notice that, even though the best fitting level for the RRA coefficient for the ultimate consumption risk model is smaller than 10 $(\hat{\gamma}=1.5)$, and at this value of the coefficient the model is able to explain about $60 \%$ of the cross-sectional variation in returns across the 25 Fama-French portfolios, all the bounds reject the model for low RRA, and the $\Psi$ bounds are not satisfied for any level of RRA. This stresses the power of the proposed approach.

The above results indicate that our entropy bounds are not only theoretically tighter but also are empirically tighter than the $\mathrm{HJ}$ variance bounds. Using the cumulants decomposition introduced in the previous section, we can identify the information content added by taking into account higher moments of the 

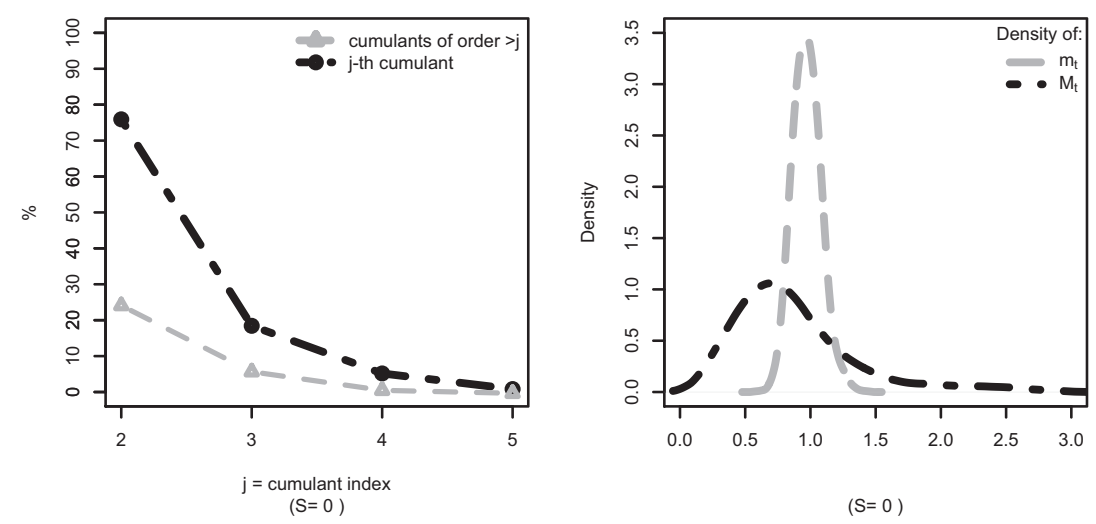

Figure 3

The relative contribution of the cumulants of $\psi_{t}^{*}$ to $D\left(P \| \Psi^{*}\right)$ is plotted $(A)$. The densities of $m_{t}:=\left(\frac{C_{t}}{C_{t-1}}\right)^{-\gamma}$ and $M_{t}^{*}:=\left(\frac{C_{t}}{C_{t-1}}\right)^{-\gamma} \psi_{t}^{*}$ are plotted $(B) . \psi_{t}^{*}$ is estimated using the relative entropy minimization procedure in Equation 6, using quarterly data for 1947:Q1-2009:Q4 and the 25 Fama-French portfolios as test assets, for the standard CCAPM with $\gamma=10$.

SDF and the components of SDF. In particular, the statistics in Equations 17] (dashed-dotted line) and (18) (dashed line) are plotted in the left panels of Figures 3 (for $S=0$ ) and 4 (for $S=11$ ).

The figures show that the contribution of the second moment to $D\left(P \| \Psi^{*}\right)$ is large - in the $74 \%-78 \%$ range - but that higher moments also play a very important role, with their cumulated contribution in the $22 \%-26 \%$ range. Among these higher moments, the lion's share goes to skewness, with individual contribution of skewness about $18 \%$ for both $S=0$ and $S=11$.

The relevance of skewness is also outlined in the right panels of Figures 3 (for $S=0$ ) and 4 (for $S=11$ ), where the (Epanechnikov kernel estimates of the) densities of $m_{t}:=\left(\frac{C_{t+S}}{C_{t-1}}\right)^{-10} R_{t, t+S}^{f}$ and $M_{t}^{*}:=\left(\frac{C_{t+S}}{C_{t-1}}\right)^{-10} R_{t, t+S}^{f} \psi_{t}^{*}$ are reported. The figures illustrate, besides the increase in variance generated by $\psi^{*}$, a substantial increase in the skewness of our estimated pricing kernel. This point is also outlined in Figures 5 (for $S=0$ ) and 6 (for $S=11$ ), where the left panels report the cumulant decomposition of the entropy of $m_{t}:=\left(\frac{C_{t+s}}{C_{t-1}}\right)^{-10} R_{t, t+S}^{f}$, while the right panel reports the cumulant decomposition for $M_{t}^{*}:=m_{t} \psi_{t}^{*}$. The figures show that the sources of entropy of our filtered pricing kernel $\left(m_{t} \psi_{t}^{*}\right)$ are very different than the ones for the consumption growth component alone $\left(m_{t}\right)$ : almost all (99\%) the entropy of $m_{t}$ is generated by its second moment, while higher cumulants have basically no role; instead, about a quarter $(24 \%-25 \%)$ of the entropy of $m_{t} \psi_{t}^{*}$ is generated by the third and higher cumulants.

We now turn to the analysis of the time-series properties of the candidate SDFs considered. Figure 7 panel A, plots the time series of the filtered 

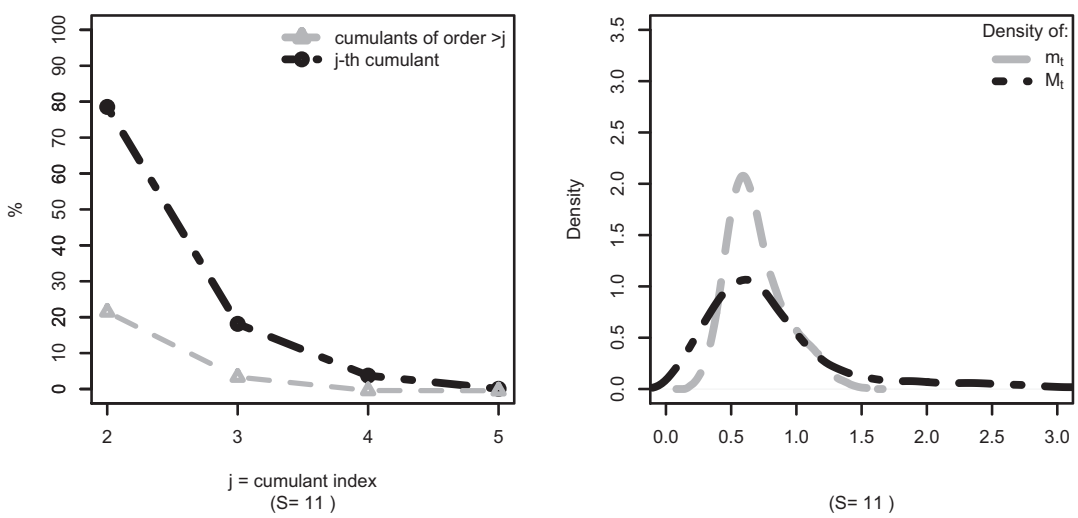

Figure 4

The relative contribution of the cumulants of $\psi_{t}^{*}$ to $D\left(P \| \Psi^{*}\right)$ is plotted $(A)$. The densities of $m_{t}:=$ $\left(\frac{C_{t+S}}{C_{t-1}}\right)^{-\gamma} R_{t, t+S}^{f}$ and $M_{t}^{*}:=\left(\frac{C_{t+S}}{C_{t-1}}\right)^{-\gamma} R_{t, t+S}^{f} \psi_{t}^{*}$ are plotted $(B) . \psi_{t}^{*}$ is estimated using the relative entropy minimization procedure in Equation [6, using qarterly data for 1947:Q1-2009:Q4 and the 25 Fama-French portfolios as test assets, for the ultimate consumption risk CCAPM of Parker and Julliard 2005) with $S=11$ and $\gamma=10$.

Cumulants contribution to the entropy of $\mathrm{m}$

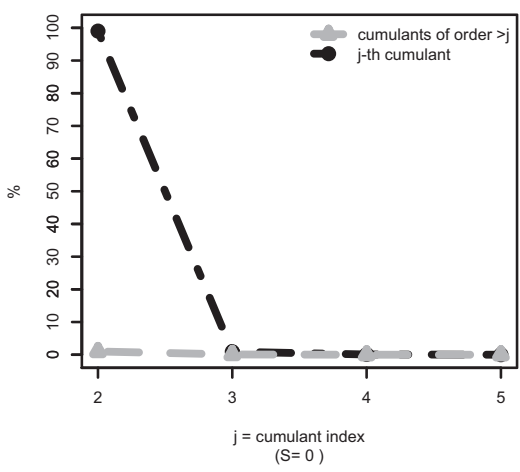

Cumulants contribution to the entropy of $M^{*}$

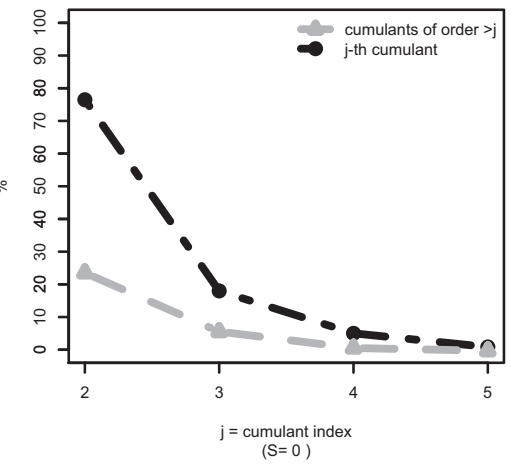

Figure 5

The contribution of the cumulants of $\left(\frac{C_{t}}{C_{t-1}}\right)^{-\gamma}$ to $D\left(P \|\left(\frac{C_{t}}{C_{t-1}}\right)^{-\gamma}\right)$ is plotted (A). The contribution of the cumulants of $\left(\frac{C_{t}}{C_{t-1}}\right)^{-\gamma} \psi_{t}^{*}$ to $D\left(P \|\left(\frac{C_{t}}{C_{t-1}}\right)^{-\gamma} \psi_{t}^{*}\right)$ is plotted $(B) . \psi_{t}^{*}$ is estimated using the relative entropy minimization procedure in Equation 6, using qarterly data for 1947:Q1-2009:Q4 and the 25 FamaFrench portfolios as test assets, for the standard CCAPM with $\gamma=10$.

SDF and the components of SDF estimated using Equation (6) for $\gamma=10$ for the contemporaneous consumption risk model $(S=0)$. The dashed line plots the component of the SDF that is a parametric function of consumption 
Cumulants contribution to the entropy of $\mathrm{m}$

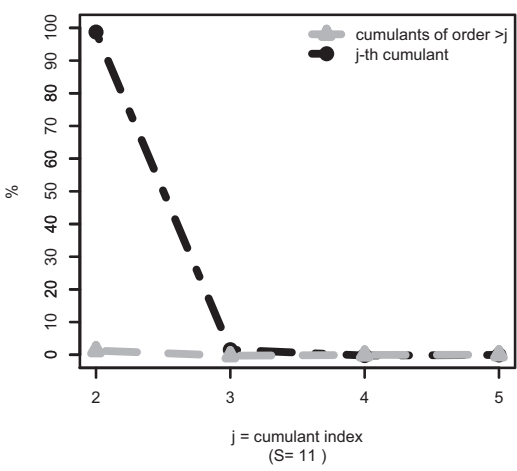

Cumulants contribution to the entropy of $\mathbf{M}^{*}$

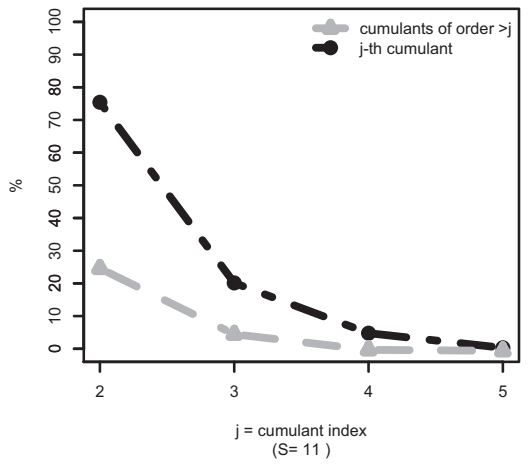

Figure 6

The contribution of the cumulants of $\left(\frac{C_{t+S}}{C_{t-1}}\right)^{-\gamma} R_{t, t+S}^{f}$ to $D\left(P \|\left(\frac{C_{t+S}}{C_{t-1}}\right)^{-\gamma} R_{t, t+S}^{f}\right)$ is plotted (A). The cumulants of $\left(\frac{C_{t+S}}{C_{t-1}}\right)^{-\gamma} R_{t, t+S}^{f} \psi_{t}^{*}$ to $D\left(P \|\left(\frac{C_{t+S}}{C_{t-1}}\right)^{-\gamma} R_{t, t+S}^{f} \psi_{t}^{*}\right)$ is plotted $(B) . \psi_{t}^{*}$ is estimated using the relative entropy minimization procedure in Equation 6, using qarterly data for 1947:Q1-2009:Q4 and the 25 Fama-French portfolios as test assets, for the ultimate consumption risk CCAPM of Parker and Julliard 2005) with $S=11$ and $\gamma=10$.

growth, $m(\theta, t)=\left(C_{t} / C_{t-1}\right)^{-\gamma}$. The dotted line with circles plots the filtered unobservable component of the SDF, $\psi_{t}^{*}$, estimated using Equation 6 . The black solid line plots the filtered SDF, $M_{t}^{*}=\left(C_{t} / C_{t-1}\right)^{-\gamma} \psi_{t}^{*}$. The gray shaded areas represent NBER-dated recessions, and the dashed-dotted vertical lines correspond to the major stock market crashes identified in Mishkin and White 2002) 15 The figure reveals two main points. First, the estimated SDF has a clear business-cycle pattern, but also shows significant and sharp reactions to financial market crashes that do not result in economy-wide contractions. Second, the time series of the SDF almost coincides with that of the unobservable component. In fact, the correlation between the two time series is 0.996 . The observable consumption growth component of the SDF, on the other hand, has a correlation of only 0.06 with the SDF. Therefore, most of the variation in the SDF comes from variation in the unobservable component, $\psi$, and not from the consumption growth component. In fact, the volatility of the SDF and its unobservable component are very similar, with the latter explaining about $99 \%$ of the volatility of the former, while the volatility of the consumption growth component accounts for only about $1 \%$ of the volatility of the filtered SDF. Similar results are obtained in panel B that plots the time series

15 Mishkin and White 2002) identify a stock market crash as a period in which the Dow Jones Industrial, the S\&P 500 , or the NASDAQ index drops by at least $20 \%$ in a time window of either one day, five days, one month, three months, or one year. Consequently, in yearly figures, we classify a given year as having a stock market crash if any such event was recorded in that year. Similarly, in quarterly figures, we identify a given quarter as being a crash period if either a crash was registered in that quarter or the entire year (containing the quarter) was identified by Mishkin and White as a stock market crash year. 
Panel A

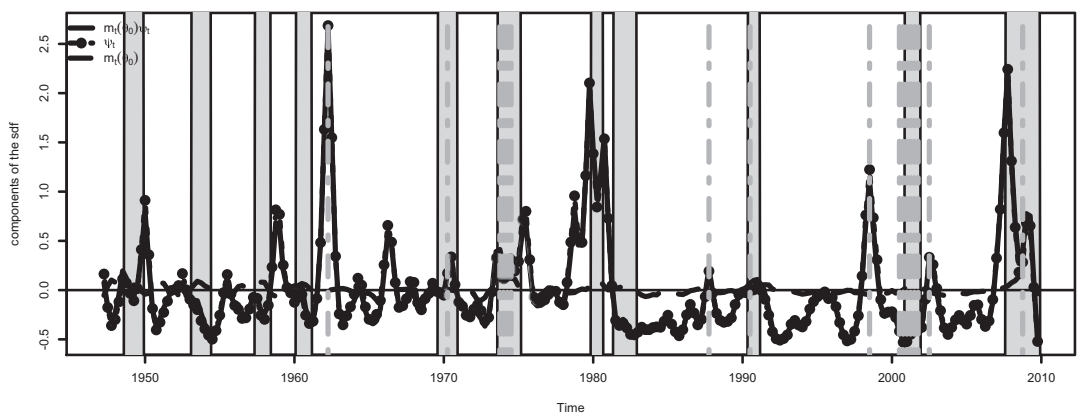

Panel B

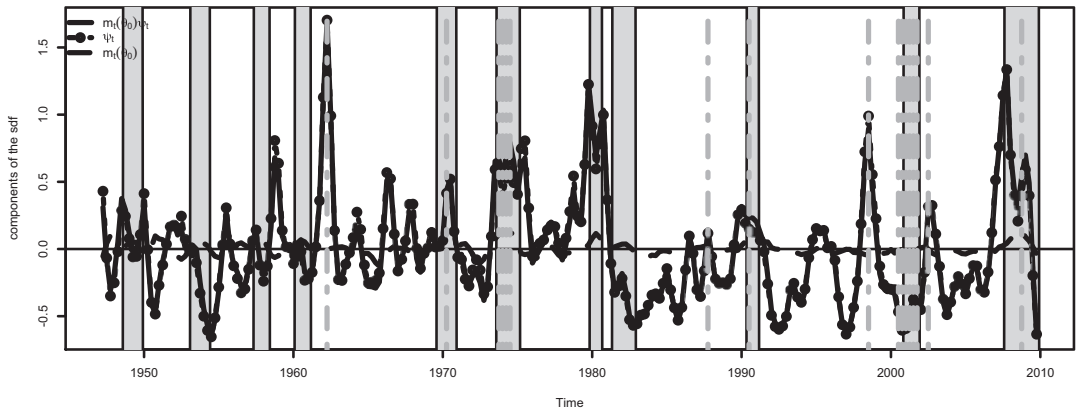

Figure 7

The figure plots the (demeaned) time series of the filtered SDF, $M_{t}^{*}=m(\theta ; t) \psi_{t}^{*}$, and its components for the standard CCAPM for $\gamma=10$. Results when $\psi_{t}^{*}$ is estimated using the relative entropy minimization procedures in Equations 6 and 4, respectively, using qarterly data for 1947:Q1-2009:Q4 and the 25 Fama-French portfolios as test assets are shown $(A$ and $B)$. Shaded areas are NBER recession periods. Vertical dot-dashed lines are the stock market crashes identified by Mishkin and White 2002).

of the filtered SDF and the components of SDF estimated using Equation (4) for $\gamma=10$.

Finally, Figure 8 panel A, plots the time series of the filtered SDF and the components of SDF estimated using Equation (6) for $\gamma=10$ for the ultimate consumption risk model $(S=11)$. The figure shows that, as in the contemporaneous consumption risk model, the estimated SDF has a clear business-cycle pattern, but also shows significant and sharp reactions to financial market crashes that do not result in economy-wide contractions. However, different from the latter model, the time series of the consumption growth component is much more volatile and more highly correlated with the SDF. The volatility of the consumption growth component is $21.7 \%$, more than 2.5 times higher than that for the standard model. The correlation between the filtered SDF and the consumption growth component of the SDF is 0.37, an order of magnitude larger than the correlation of 0.06 in the contemporaneous consumption risk model. This explains the ability of the model to account for 
Panel A

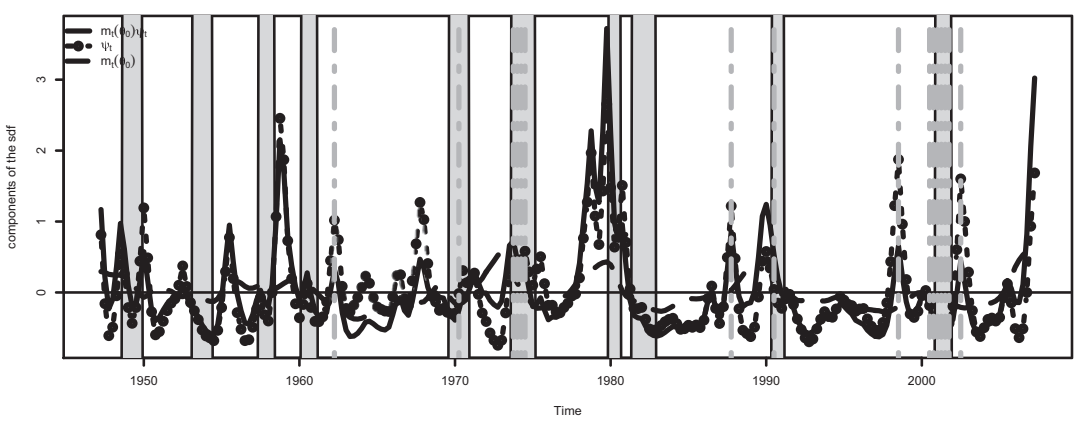

Panel B

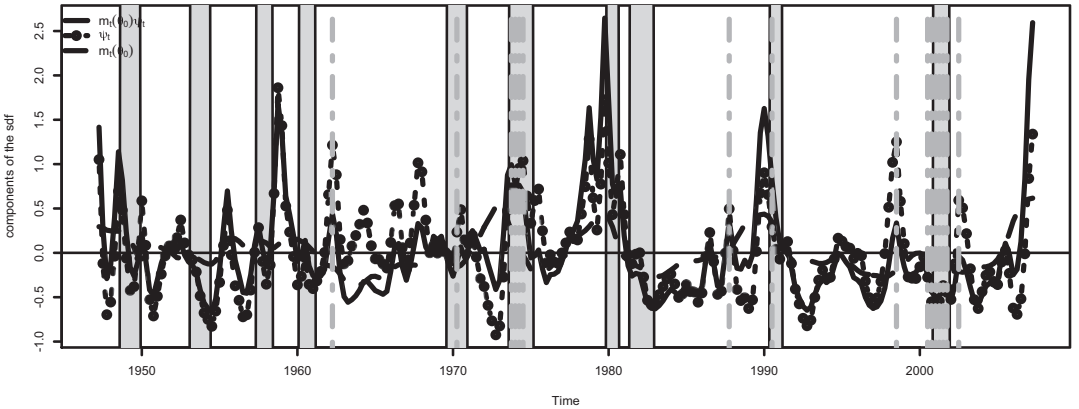

Figure 8

The figure plots the (demeaned) time series of the filtered SDF, $M_{t}^{*}=m(\theta ; t) \psi_{t}^{*}$, and its components for the ultimate consumption risk CCAPM of Parker and Julliard 2005) for $\gamma=10$. Results when $\psi_{t}^{*}$ is estimated using the relative entropy minimization procedures in Equations 6 and 4, respectively, using qarterly data for 1947:Q1-2009:Q4 and the 25 Fama-French portfolios as test assets are shown ( $A$ and $B$ ). Shaded areas are NBER recession periods. Vertical dot-dashed lines are the stock market crashes identified by Mishkin and White 2002.

a much larger fraction of the variation in expected returns across the 25 FamaFrench portfolios for low levels of the risk-aversion coefficient. In fact, the cross-sectional $R^{2}$ of the model is $54.1 \%$ (for $\gamma=10$ ), an order of magnitude higher than the value of $5.2 \%$ for the standard model. However, the correlation between the ultimate consumption risk SDF and the unobservable component of the SDF is still very high at 0.92 , showing that the model is missing important elements that would further improve its ability to explain the cross-section of returns. Similar results are obtained in panel B that plots the time series of the filtered SDF and the components of the SDF estimated using Equation (4) for $\gamma=10$.

Overall, the results show that our methodology provides useful diagnostics for dynamic asset pricing models. Moreover, the very similar results obtained using the two different types of relative entropy minimization in Equations (4) and (6) suggest robustness of our approach. 


\section{Application to More General Models of Dynamic Economies}

Our methodology provides useful diagnostics to assess the empirical plausibility of a large class of consumption-based asset pricing models in which the SDF, $M_{t}$, can be factorized into an observable component consisting of a parametric function of consumption, $C_{t}$, as in the standard time-separable power utility model and a potentially unobservable one, $\psi_{t}$, that is modelspecific. In this section, we apply it to a set of "winner" asset pricing models, that is, frameworks that can successfully explain the equity premium and the risk-free rate puzzles with "reasonable" calibrations. In particular, we consider the external habit formation models of Campbell and Cochrane 1999) and Menzly et al. 2004, the long-run risks model of Bansal and Yaron 2004), and the housing model of Piazzesi et al. 2007). We apply our methodology to assess the empirical plausibility of these models in two ways. First, since our approach delivers an estimate of the time series of the SDF, for each model considered we compare the estimated time series with the model-implied one. Second, for each model we compute the values of the power coefficient, $\gamma$, at which the model-implied SDF satisfies the $H J, Q, M$, and $\Psi$ bounds.

In the next subsection we present the models considered. The reader familiar with these models can go directly to Section 3.2 which reports the empirical results, without loss of continuity. A detailed data description is presented in Appendix A.4

\subsection{The models considered}

3.1.1 External habit formation model: Campbell and Cochrane (1999). In this model, identical agents maximize power utility, defined over the difference between consumption and a slow-moving habit or time-varying subsistence level. The SDF is given by

$$
M_{t}^{m}=\underbrace{\left(C_{t} / C_{t-1}\right)^{-\gamma}}_{m(\theta, t)} \underbrace{\delta\left(S_{t} / S_{t-1}\right)^{-\gamma}}_{\psi_{t}^{m}},
$$

where $\delta$ is the subjective time discount factor, $\gamma$ is the curvature parameter that provides a lower bound on the time-varying coefficient of relative-risk aversion, $S_{t}=\frac{C_{t}-X_{t}}{C_{t}}$ denotes the surplus consumption ratio, and $X_{t}$ is the habit component. Note that the $\psi^{m}$ component depends on the surplus consumption ratio, $S$, not directly observed. To obtain the time series of $\psi^{m}$, we extract the surplus consumption ratio from observed data using two different procedures.

First, we extract the time series of the surplus consumption ratio from consumption data. In this model, the aggregate consumption growth is assumed to follow an i.i.d. process:

$$
\Delta c_{t}=g+v_{t}, \quad v_{t} \sim \text { i.i.d.N }\left(0, \sigma^{2}\right) .
$$

The log surplus consumption ratio evolves as a heteroscedastic $A R(1)$ process:

$$
s_{t}=(1-\phi) \bar{s}+\phi s_{t-1}+\lambda\left(s_{t-1}\right) v_{t},
$$


where $s_{t}:=\ln S_{t}$ and $\bar{s}$ is the steady-state $\log$ surplus consumption ratio and

$$
\begin{gathered}
\lambda\left(s_{t}\right)=\left\{\begin{array}{cc}
\frac{1}{\bar{S}} \sqrt{1-2\left(s_{t}-\bar{s}\right)}-1, & \text { if } s_{t} \leq s_{\max } \\
0, & \text { if } s_{t}>s_{\text {max }}
\end{array},\right. \\
s_{\text {max }}=\bar{s}+\frac{1}{2}\left(1-\bar{S}^{2}\right), \quad \bar{S}=\sigma \sqrt{\frac{\gamma}{1-\phi}} .
\end{gathered}
$$

For each value of $\gamma$, we use the calibrated values of the model preference parameters $(\delta, \phi)$ in Campbell and Cochrane 1999), the sample mean $(g)$ and volatility $(\sigma)$ of the consumption growth process, and the innovations in real consumption growth, $\widehat{v}_{t}=\Delta c_{t}-g$, to extract the time series of the surplus consumption ratio using Equation 22 and, thereby, obtain the time series of the model-implied SDF and its $\psi^{m}$ component.

Second, in this model, the equilibrium market-wide price-dividend ratio is a function of the surplus consumption ratio alone, although the form of the function is not available in closed form. Using numerical methods, we invert this function to extract the time series of the surplus consumption ratio from the historical time series of the price-dividend ratio and thereby obtain the time series of the model-implied SDF and its $\psi^{m}$ component from Equation 21.

3.1.2 External habit formation model: Menzly et al. (2004). In this model, the SDF is analogous to the Campbell and Cochrane 1999) one disussed above. The aggregate consumption growth is also assumed to follow an i.i.d. process:

$$
d c_{t}=\mu_{c} d t+\sigma_{c} d B_{t}
$$

where $\mu_{c}$ is the mean consumption growth, $\sigma_{c}>0$ is a scalar, and $B_{t}$ is a Brownian motion. The point of departure from the Campbell and Cochrane 1999) framework is that Menzly et al. 2004 assume that the inverse surplus consumption ratio, $Y_{t}:=\frac{1}{S_{t}}$, follows a mean-reverting process perfectly negatively correlated with innovations in consumption growth:

$$
d Y_{t}=k\left(\bar{Y}-Y_{t}\right) d t-\alpha\left(Y_{t}-\lambda\right)\left[d c_{t}-E\left(d c_{t}\right)\right],
$$

where $\bar{Y}$ is the long-run mean of the inverse surplus consumption ratio and $k$ controls the speed of mean reversion. To obtain the time series of $\psi^{m}$ (the model-implied $\psi$ component), we extract the surplus consumption ratio from observed data using two different procedures.

First, for each value of $\gamma / 16$ we use the calibrated values of the model parameters $(\delta, k, \bar{Y}, \alpha, \lambda)$ in Menzly et al. 2004), the sample values of $\mu_{c}$

16 Note that the Menzly et al 2004 model assumes that the representative agent has log utility, that is, $\gamma$ is set equal to 1 , to derive the closed-form solution for the price-consumption ratio. For other values of $\gamma$, the model does not admit a closed-form solution. Nevertheless, the pricing kernel is well defined even if $\gamma$ is different than one; hence we will be considering this more general case. 
and $\sigma_{c}$, and the innovations in real consumption growth, $\widehat{d B_{t}}=\frac{\left[d c_{t}-E\left(d c_{t}\right)\right]}{\sigma_{c}}$, to extract the time series of the surplus consumption ratio, and this allows us to compute the time series of the model-implied SDF.

Second, in this model, the equilibrium price-consumption ratio of the total wealth portfolio is a function of the surplus consumption ratio alone. However, this function is not available in closed form except for $\gamma=1$. Therefore, we rely on log-linear approximations to the return on the total wealth portfolio to express the equilibrium $\log$ price-consumption ratio as an affine function of the $\log$ surplus consumption ratio for all values of $\gamma$. Details of this procedure are described in Appendix A.5 We then invert this affine function to extract the time series of the surplus consumption ratio from the historical time series of the market-wide price-dividend ratio and thereby obtain the time series of the model-implied SDF and its $\psi^{m}$ component from Equation 21. Note that approximating the total wealth price-consumption ratio by the market-wide price-dividend ratio is the approach used by Menzly et al. 2004).

3.1.3 Long-run Risks model: Bansal and Yaron (2004). Bansal and Yaron's (2004) long-run risks model assumes that the representative consumer has the version of Kreps and Porteus 1978) preferences adopted by Epstein and Zin 1989) and Weil (1989), for which the SDF is given by

$$
M_{t+1}^{m}=\delta^{\theta}\left(\frac{C_{t+1}}{C_{t}}\right)^{-\frac{\theta}{\rho}} R_{c, t+1}^{\theta-1},
$$

where $R_{c, t+1}$ is the unobservable gross return on an asset that delivers aggregate consumption as its dividend each period, $\delta$ is the subjective time discount factor, $\rho$ is the elasticity of intertemporal substitution, $\theta:=\frac{1-\gamma}{1-1 / \rho}$, and $\gamma$ is the relative-risk aversion coefficient.

The aggregate consumption and dividend growth rates, $\Delta c_{t+1}$ and $\Delta d_{t+1}$, respectively, are modeled as containing a small persistent expected growth rate component, $x_{t}$, which follows an AR(1) process with stochastic volatility, and fluctuating variance, $\sigma_{t}^{2}$, which evolves according to a homoscedastic linear mean-reverting process.

Appendix A.6 shows that, for the log-linearized model, the log of the SDF and its $\psi^{m}$ component are given by

$$
\ln M_{t+1}^{m}=\underbrace{c_{2} \Delta c_{t+1}}_{\ln m(\theta, t+1)}+\underbrace{c_{1}+c_{3} x_{t+1}+c_{4} \sigma_{t+1}^{2}+c_{5} x_{t}+c_{6} \sigma_{t}^{2}}_{\ln \psi_{t+1}^{m}}
$$

where the parameters $\left(c_{1}, c_{2}, c_{3}, c_{4}, c_{5}, c_{6}\right)$ are known functions of the underlying time series and preference parameters of the model.

To obtain the time series of the SDF and $\psi^{m}$, we extract the state variables, $x_{t}$ and $\sigma_{t}^{2}$, from observed data using two different procedures. First, we extract them from consumption data. Second, we extract them from asset market data, in particular, from the market-wide price-dividend ratio and the risk-free rate. 
The extraction of the state variables using these two procedures is described in Appendix A.6 Finally, for each value of $\gamma$, we use the calibrated parameter values from Bansal and Yaron 2004) and the time series of the state variables to obtain the time series of the SDF and its $\psi^{m}$ component from Equation (24).

3.1.4 Housing: Piazzesi et al. (2007). In this model, the pricing kernel is given by

$$
M_{t}^{m}=\delta\left(C_{t} / C_{t-1}\right)^{-\gamma}\left(A_{t} / A_{t-1}\right)^{\frac{\gamma \rho-1}{\rho-1}},
$$

where $A_{t}$ is the expenditure share on nonhousing consumption, $\gamma^{-1}$ is the intertemporal elasticity of substitution, and $\rho$ is the intratemporal elasticity of substitution between housing services and nonhousing consumption.

Taking the logs, we have

$$
\ln M_{t}^{m}=\underbrace{-\gamma \Delta c_{t}}_{\ln m(\theta, t)}+\underbrace{\ln \delta+\frac{\gamma \rho-1}{\rho-1} \Delta a_{t}}_{\ln \psi_{t}^{m}} .
$$

Note that, in this model, $\psi^{m}$ depends on observable variables alone and, therefore, does not need to be extracted from consumption or asset market data. For each value of $\gamma$, we use the calibrated values of the model parameters $(\delta, \rho)$ in Piazzesi et al 2007) to obtain the time series of the model-implied SDF and its $\psi^{m}$ component from Equation 25 .

\subsection{Empirical results}

For our empirical analysis, we focus on two data samples: an annual data sample starting at the onset of the Great Depression (1929-2009), and a quarterly data sample starting in the post-World War II period (1947: $Q 1-2009: Q 4)$. A detailed data description is presented in Appendix A.4 Note that, in any finite sample, the extracted time series of the SDF, as well as the information bounds on the SDF and the unobservable component of the SDF, depend on the set of test assets used for their construction. Since the Euler equation holds for any traded asset, as well as for any adapted portfolio of assets, this gives an infinitely large number of moment restrictions. Nevertheless, econometric considerations necessitate the choice of only a subset of assets to be used. As a consequence, in our empirical analysis, we compute bounds and filter the time series of the SDF and the components of the SDF, using a broad crosssection of test assets. In particular, at the quarterly frequency, the test assets include the 6 size and book-to-market-equity-sorted portfolios of Fama-French, 10 industry-sorted portfolios, and 10 momentum-sorted portfolios. Due to the smaller available time series at the annual frequency, we restrict the crosssection of test assets to include the 6 size and book-to-market-equity-sorted portfolios, 5 industry-sorted portfolios, and the smallest and largest deciles of the 10 momentum-sorted portfolios. 
Table 1

Correlation of filtered and model SDFs, 1947:Q1-2009:Q4

\begin{tabular}{|c|c|c|c|c|}
\hline & \multicolumn{2}{|c|}{$\begin{array}{l}\text { Correlation of filtered } \\
\text { and model SDF }\end{array}$} & \multicolumn{2}{|c|}{$\begin{array}{c}\text { Cross-sectional } \\
R^{2}\end{array}$} \\
\hline & $\rho\left(\ln \psi_{t}^{*}, \ln \psi_{t}^{m}\right)$ & $\rho\left(\ln M_{t}^{*}, \ln M_{t}^{m}\right)$ & $\begin{array}{c}\text { No } \\
\text { intercept }\end{array}$ & $\begin{array}{c}\text { Free } \\
\text { intercept }\end{array}$ \\
\hline \multicolumn{5}{|c|}{ A. State variables extracted from consumption } \\
\hline$\overline{C C}$ & $\begin{array}{c}0.10 / 0.07 \\
{[-0.09,0.18][-0.11,0.18]}\end{array}$ & $\begin{array}{c}0.13 / 0.09 \\
{[-0.07,0.20][-0.09,0.19]}\end{array}$ & $\begin{array}{c}-1.19 \\
{[-3.14,0.02]}\end{array}$ & $\begin{array}{c}0.002 \\
{[0.00,0.38]}\end{array}$ \\
\hline$M S V$ & $\begin{array}{c}-0.01 / 0.003 \\
{[-0.07,0.18][-0.09,0.18]}\end{array}$ & $\begin{array}{c}0.05 / 0.04 \\
{[-0.07,0.20][-0.09,0.19]}\end{array}$ & $\begin{array}{c}-0.79 \\
{[-2.72,0.06]}\end{array}$ & $\begin{array}{c}0.002 \\
{[0.000,0.37]}\end{array}$ \\
\hline$B Y$ & $\begin{array}{c}-0.02 / 0.03 \\
{[-0.14,0.12][-0.11,0.18]}\end{array}$ & $\begin{array}{c}0.16 / 0.09 \\
{[-0.03,0.25][-0.12,0.18]}\end{array}$ & $\begin{array}{c}-0.71 \\
{[-2.83,0.02]}\end{array}$ & $\begin{array}{c}0.005 \\
{[0.00,0.35]}\end{array}$ \\
\hline$P S T$ & $\begin{array}{c}-0.12 /-0.14 \\
{[-0.24,0.02][-0.24,0.03]}\end{array}$ & $\begin{array}{c}-0.03 /-0.04 \\
{[-0.16,0.09][-0.21,0.09]}\end{array}$ & $\begin{array}{c}-0.91 \\
{[-3.21,0.14]}\end{array}$ & $\begin{array}{c}0.03 \\
{[0.00,0.36]}\end{array}$ \\
\hline \multicolumn{5}{|c|}{ B. State variables extracted from asset prices } \\
\hline$\overline{C C}$ & $\begin{array}{c}0.17 / 0.16 \\
{[-0.10,0.18][-0.10,0.18]}\end{array}$ & $\begin{array}{c}0.18 / 0.17 \\
{[-0.10,0.18][-0.10,0.19]}\end{array}$ & $\begin{array}{c}-0.77 \\
{[-3.13,0.08]}\end{array}$ & $\begin{array}{c}0.31 \\
{[0.00,0.39]}\end{array}$ \\
\hline$M S V$ & $\begin{array}{c}0.18 / 0.23 \\
{[-0.10,0.19][-0.10,0.22]}\end{array}$ & $\begin{array}{c}0.19 / 0.24 \\
{[-0.10,0.20][-0.10,0.22]}\end{array}$ & $\begin{array}{c}-0.46 \\
{[-3.78,0.00]}\end{array}$ & $\begin{array}{c}0.04 \\
{[0.00,0.48]}\end{array}$ \\
\hline$B Y$ & $\begin{array}{c}0.03 / 0.06 \\
{[-0.11,0.17][-0.11,0.21]}\end{array}$ & $\begin{array}{c}0.04 / 0.07 \\
{[-0.11,0.17][-0.10,0.21]}\end{array}$ & $\begin{array}{c}-1.26 \\
{[-3.23,-0.39]}\end{array}$ & $\begin{array}{c}0.24 \\
{[0.00,0.52]}\end{array}$ \\
\hline
\end{tabular}

The table reports the correlation between the filtered and the model-implied $\psi$-components of the SDFs (Column 1), the correlation between the filtered and the model-implied SDFs (Column 2), the cross-sectional $\mathrm{R}^{2}$ implied by the model-specific SDFs when no intercept is allowed in the cross-sectional regression (Column 3), and the cross-sectional $\mathrm{R}^{2}$ when an intercept is allowed in the regression (Column 4), using quarterly data for 1947:Q1-2009:Q4. The bootstrapped 95\% confidence intervals are reported in brackets. Each cell in Columns 1 and 2 has two entries corresponding to whether the filtered $\psi^{*}$-component and, therefore, the filtered SDF is estimated using Equation 6, reported on the left, or Equation 44, reported on the right. Panel A reports results when the models' state variables and, therefore, the model-implied SDFs are extracted from consumption data, and panel B reports the same when the state variables are extracted from asset prices. $C C, M S V, B Y$, and $P S T$ denote, respectively, the models of Campbell and Cochran 1999, Menzly et al, 2004, Bansal and Yaron 2004, and Piazzesi et al. 2007).

3.2.1 The time series of the filtered SDF. Our first approach for assessing the empirical plausibility of these models is based on the observation that our method identifies the minimum entropy time series of the SDF, which we call the filtered SDF. That is, given a candidate SDF with observable component $m(\theta, t)$, we use the relative entropy-minimizing procedures in Equations (4) and (6) to estimate a time series for the unobservable (or residual, if the SDF is fully observable) component $\left\{\psi_{t}^{*}(\theta)\right\}_{t=1}^{T}$ and obtain the filtered SDF as $m(\theta, t) \psi_{t}^{*}$.

Note that the filtered SDF and its missing component depend on the local curvature of the utility function $\gamma$, since changing $\gamma$ modifies the constraints in Equations (4) and (6). Therefore, for each model, we fix $\gamma$ at the authors' calibrated value and extract the time series of the filtered SDF and the components of the SDF. We compare the filtered $\operatorname{SDF}\left(m(\theta, t) \psi_{t}^{*}\right)$ with the model-implied SDF $\left(m(\theta, t) \psi_{t}^{m}\right)$ for each model.

Table 1 reports the results at the quarterly frequency. Panel A reports results when the model-implied SDF and its components are obtained by extracting the state variable(s) from consumption data, and panel B presents results when asset market data are used to extract the state variable(s). The first column reports 
the correlation between the filtered time series of the missing component, $\left\{\ln \psi_{t}^{*}\right\}_{t=1}^{T}$, of the SDF, and of the corresponding model-implied time series, $\left\{\ln \psi_{t}^{m}\right\}_{t=1}^{T}$. The second column shows the correlation between the filtered $\mathrm{SDF},\left\{\ln M_{t}^{*}=\ln \left(m(\theta, t) \psi_{t}^{*}\right)\right\}_{t=1}^{T}$, where $m(\theta, t)=\left(C_{t} / C_{t-1}\right)^{-\gamma}$, and the modelimplied SDF, $\left\{\ln M_{t}^{m}=\ln \left(m(\theta, t) \psi_{t}^{m}\right)\right\}_{t=1}^{T}$. The $95 \%$ confidence intervals for these correlations are obtained by bootstrapping with replacement from the data.

Consider first the results for the CC external habit model presented in the first row of each panel. For this model, the utility curvature parameter is set to the calibrated value of $\gamma=2$. Column 1 in panel A shows that when the modelimplied state variable is extracted from consumption, the correlation between the filtered and model-implied $\psi$ is only 0.10 when $\psi^{*}$ is estimated using Equation (6). Column 2 shows that the correlation between the filtered and model-implied SDFs is marginally higher at 0.13 . When $\psi^{*}$ is estimated using Equation (4), the correlations are very similar at 0.07 and 0.09 , respectively. Panel B shows that the correlations between the filtered and model-implied SDFs and $\psi$ 's remain small when the model state variable is extracted from the market-wide price-dividend ratio.

The second row in each panel presents the results for the MSV external habit model. In this case, $\gamma$ is set equal to 1 , which is the calibrated value in the model. Row 2 in each panel shows that the results for the MSV model are similar to those for the CC model. When $\psi^{*}$ is estimated using Equation (6), the correlations between the filtered and model-implied $\psi$ components of the SDFs are small, varying from -0.01 , when the surplus consumption ratio is extracted from consumption data, to 0.18 , when the state variable is extracted using the price-dividend ratio. The correlations between the filtered and model-implied SDFs are marginally higher, varying from 0.05 , when the surplus consumption ratio is extracted from consumption data, to 0.19 when it is extracted using the price-dividend ratio. Similar results are obtained when $\psi^{*}$ is estimated using Equation (4).

The third row in each panel presents the results for the BY long-run risks model. The parameter $\gamma$ is set equal to the BY calibrated value of 10 . Row 3 and Column 1 of panel $\mathrm{A}$, shows that when the state variables are extracted from consumption, the correlation between the filtered and model-implied $\psi$ components is $-0.02(0.03)$ when $\psi^{*}$ is estimated using Equation (6) (Equation (4)). Column 2 shows that the correlation between the filtered and model-implied SDFs is 0.16 (0.09). Similar results are obtained in panel B, where the state variables are extracted from the market-wide price-dividend ratio.

The fourth row in panel A presents the results for the PST housing model. Note that, in this model, the SDF and its $\psi^{m}$ component are directly observable and thereby do not need to be extracted from either consumption or asset market data. Therefore, we do not have a fourth row in panel B. The risk-aversion parameter, $\gamma$, is set equal to 16 , which is the calibrated value in the original 
Table 2

Correlation of filtered and model SDFs, 1929-2009

\begin{tabular}{|c|c|}
\hline $\begin{array}{l}\text { Correlation of filtered } \\
\text { and model SDF }\end{array}$ & $\begin{array}{c}\text { Cross-sectional } \\
R^{2}\end{array}$ \\
\hline$\rho\left(\ln M_{t}^{*}, \ln M_{t}^{m}\right)$ & $\begin{array}{c}\text { No } \\
\text { intercept }\end{array}$ \\
\hline
\end{tabular}

\begin{tabular}{|c|c|c|c|c|}
\hline \multicolumn{5}{|c|}{ A. State variables extracted from consumption } \\
\hline$\overline{C C}$ & $\begin{array}{c}0.35 / 0.31 \\
{[-0.04,0.44][-0.04,0.41]}\end{array}$ & $\begin{array}{c}0.39 / 0.34 \\
{[-0.00,0.48][-0.02,0.92]}\end{array}$ & $\begin{array}{c}0.082 \\
{[-2.19,0.74]}\end{array}$ & $\begin{array}{c}0.504 \\
{[0.00,0.81]}\end{array}$ \\
\hline$M S V$ & $\begin{array}{c}0.33 / 0.22 \\
{[-0.02,0.41][-0.04,0.37]}\end{array}$ & $\begin{array}{c}0.41 / 0.34 \\
{[0.06,0.46][-0.02,0.96]}\end{array}$ & $\begin{array}{c}0.76 \\
{[-2.05,0.76]}\end{array}$ & $\begin{array}{c}0.82 \\
{[0.00,0.80]}\end{array}$ \\
\hline$B Y$ & $\begin{array}{c}-0.17 /-0.028 \\
{[-0.31,0.22][-0.44,0.21]}\end{array}$ & $\begin{array}{c}0.27 / 0.20 \\
{[-0.03,0.48][-0.16,0.77]}\end{array}$ & $\begin{array}{c}0.45 \\
{[-2.25,0.75]}\end{array}$ & $\begin{array}{c}0.47 \\
{[0.00,0.80]}\end{array}$ \\
\hline PST & $\begin{array}{c}-0.09 /-0.001 \\
{[-0.23,0.24][-0.25,0.25]}\end{array}$ & $\begin{array}{c}-0.004 /-0.013 \\
{[-0.20,0.21][-0.26,0.26]}\end{array}$ & $\begin{array}{c}-0.73 \\
{[-2.39,0.09]}\end{array}$ & $\begin{array}{c}0.09 \\
{[0.00,0.40]}\end{array}$ \\
\hline \multicolumn{5}{|c|}{ B. State variables extracted from asset prices } \\
\hline$\overline{\mathrm{CC}}$ & $\begin{array}{c}0.19 / 0.14 \\
{[-0.12,0.35][-0.10,0.28]}\end{array}$ & $\begin{array}{c}0.24 / 0.17 \\
{[-0.11,0.37][-0.08,0.29]}\end{array}$ & $\begin{array}{c}-0.20 \\
{[-2.86,0.53]}\end{array}$ & $\begin{array}{c}0.60 \\
{[0.00,0.63]}\end{array}$ \\
\hline MSV & $\begin{array}{c}-0.04 / 0.13 \\
{[-0.10,0.33][-0.10,0.27]}\end{array}$ & $\begin{array}{c}0.01 / 0.18 \\
{[-0.08,0.35][-0.09,0.28]}\end{array}$ & $\begin{array}{c}-0.16 \\
{[-2.69,0.27]}\end{array}$ & $\begin{array}{c}0.001 \\
{[0.00,0.52]}\end{array}$ \\
\hline BY & $\begin{array}{c}-0.01 / 0.10 \\
{[-0.21,0.34][-0.23,0.31]}\end{array}$ & $\begin{array}{c}-0.02 / 0.09 \\
{[-0.21,0.29][-0.29,0.32]}\end{array}$ & $\begin{array}{c}-0.15 \\
{[-0.77,0.25]}\end{array}$ & $\begin{array}{c}0.005 \\
{[0.00,0.27]}\end{array}$ \\
\hline
\end{tabular}

The table reports the correlation between the filtered and the model-implied $\psi$-components of the SDFs (Column 1), the correlation between the filtered and the model-implied SDFs (Column 2), the cross-sectional $\mathrm{R}^{2}$ implied by the model-specific SDFs when no intercept is allowed in the cross-sectional regression (Column 3 ), and the cross-sectional $R^{2}$ when an intercept is allowed in the regression (Column 4), using annual data for 1929-2009. The bootstrapped 95\% confidence intervals are reported in brackets. Each cell in Columns 1 and 2 has two entries corresponding to whether the filtered $\psi^{*}$-component and, therefore, the filtered SDF is estimated using Equation 6, reported on the left, or Equation 4, reported on the right. Panel A reports results when the models' state variables and, therefore, the model-implied SDFs are extracted from consumption data, and panel $\mathrm{B}$ reports the same when the state variables are extracted from asset prices. $C C, M S V, B Y$, and $P S T$ denote, respectively, the models of Campbell and Cochrane 1999, Menzly et al 2004, Bansal and Yaron 2004, and Piazzesi et al, 2007).

paper. Column 1 shows that the correlations between the filtered and modelimplied $\psi$ components of the SDFs are very small and have the wrong sign, varying from -0.12 to -0.14 , when $\psi^{*}$ is estimated using Equations (6) and (4). The correlations between the filtered and model-implied SDFs are marginally higher varying from -0.03 to -0.04 .

Table 2 reports results analogous to those in Table 1 at the annual frequency. The results are largely similar to those in Table1 The two habit models, when the state variable is extracted from consumption data, are notable exceptions. In these two cases the correlations between filtered and model-implied SDFs and $\psi$ components are much higher than at the quarterly frequency, being in the 0.31-0.39 range for CC and 0.22-0.41 for MSV.

The last two columns of Tables 1 and 2 report the cross-sectional $R^{2}$ 's, along with $95 \%$ confidence bands, in brackets, implied by the model-specific SDFs at the quarterly and annual frequencies, respectively. The cross-sectional $R^{2}$ are obtained by performing a cross-sectional regression of the historical average returns on the model-implied expected returns. Column 3 reports the crosssectional $R^{2}$ when there is no intercept in the regression, and Column 4 presents 
results when an intercept is included. The results reveal that the cross-sectional $R^{2}$ 's wildly vary for the same model, and often take on large negative values when an intercept is not allowed in the cross-sectional regression, or when the model-implied state variables are extracted using either consumption or asset market data. Moreover, they have very wide confidence intervals. As we show in the next subsection, this is in stark contrast with the results based on entropy bounds in Tables 6 and 7 that tend instead to give consistent results and tighter confidence bands for each model across different samples and procedures used to extract the model state variables.

Overall, Tables 1 and 2 make two main points. First, they demonstrate the robustness of our estimation method - very similar results are obtained using either Equation (4) or (6) to filter $\psi^{*}$ and $M^{*}$. Second, they show that, regardless of the data frequency and the procedure used to extract the model-implied SDFs, all the asset pricing models considered imply SDFs that tend to have low correlation with the filtered ones. While the results in Tables 1 and 2 are obtained using the combined set of size and book-to-market-equity-sorted, momentumsorted, and industry-sorted portfolios, very similar results are obtained using the 25 Fama-French portfolios as test assets 17

The correlations between model-specific SDFs and filtered SDFs discussed above would have little significance if the filtered discount factors had no clear economic interpretation. To address this concern, we show below that our filtered pricing kernel has clear economic content since (1) it is always highly correlated with the Fama-French factors, that can be interpreted as proxies for the true unknown sources of systematic risk, (2) it implies that the SDF should have a strong business-cycle pattern, and (3) it significantly reacts to financial market crashes.

Tables 3 and 4 report the correlations between the filtered and model-implied $\log$ SDFs and the three Fama-French (FF) factors at the quarterly and annual frequencies, respectively. Column 1 presents the correlation between the modelimplied SDF, when the state variables are extracted from consumption data, and the three FF factors. This is computed by performing a linear regression of the model-implied time series of the SDF, $\left\{\ln \left(M_{t}^{m}\right)\right\}_{t=1}^{T}$, on the three FF factors and computing the correlation between $\ln \left(M^{m}\right)$ and the fitted value from the regression. Column 2 reports the correlation when the model-implied state variables are extracted from asset market data. Columns 3 and 4 present the correlations of the filtered SDF and its missing component with the three FF factors, respectively.

Consider Table 3 first. Column 3, panel A, shows that the log of the filtered SDF, $M_{t}^{*} \equiv m(\theta, t) \psi_{t}^{*}$, strongly correlates with the FF factors, having correlation coefficients ranging from 0.49 to 0.59 when the set of test assets

17 The results are available from the authors on request. 
Table 3

Correlations with FF3, 1947:Q1-2009:Q4

Correlation with FF3

\begin{tabular}{lcccc}
\hline & $\left(\ln M_{t}^{m}\right)_{\text {cons }}$ & $\left(\ln M_{t}^{m}\right)_{\text {prices }}$ & $\ln M_{t}^{*}$ & $\ln \psi_{t}^{*}$ \\
\hline A. 25 Fama-French & & & & \\
\hline CC & 0.18 & 0.20 & $0.54 / 0.59$ & $0.54 / 0.59$ \\
MSV & 0.21 & 0.95 & $0.54 / 0.59$ & $0.54 / 0.59$ \\
BY & 0.25 & 0.45 & $0.54 / 0.58$ & $0.52 / 0.57$ \\
PST & 0.07 & - & $0.49 / 0.52$ & $0.45 / 0.50$ \\
\hline B. 10 Momentum & & & & \\
\hline CC & 0.18 & 0.20 & $0.52 / 0.52$ & $0.51 / 0.51$ \\
MSV & 0.21 & 0.95 & $0.52 / 0.52$ & $0.51 / 0.51$ \\
BY & 0.25 & 0.45 & $0.55 / 0.53$ & $0.50 / 0.50$ \\
PST & 0.07 & - & $0.53 / 0.51$ & $0.43 / 0.43$ \\
\hline C. 10 Industry & & & & \\
\hline CC & 0.18 & 0.20 & $0.65 / 0.69$ & $0.64 / 0.68$ \\
MSV & 0.21 & 0.95 & $0.65 / 0.69$ & $0.65 / 0.68$ \\
BY & 0.25 & 0.45 & $0.66 / 0.69$ & $0.62 / 0.65$ \\
PST & 0.07 & - & $0.53 / 0.55$ & $0.47 / 0.51$ \\
\hline Th tablepr &
\end{tabular}

The table reports the correlations between the 3 Fama-French factors and (1) the model-implied SDF with state variables extracted from consumption (Column 1) and stock market (Column 2) data, (2) the filtered SDF (Column 3 ), and (3) the filtered $\psi^{*}$ component of the SDF (Column 4), using quarterly data for 1947:Q1-2009:Q4 and a different set of portfolios in each panel. Each cell in Columns 3 and 4 has two entries corresponding to whether the filtered $\psi^{*}$-component and, therefore, the filtered SDF is estimated using Equation 6, reported on the left, or Equation 4 , reported on the right. $C C, M S V, B Y$, and PST denote, respectively, the models of Campbell and Cochrane 1999), Menzly et all 2004), Bansal and Yaron 2004), and Piazzesi et al 2007).

consists of the 25 size and book-to-market-equity-sorted portfolios of FamaFrench. Column 4 reveals that this high correlation is almost entirely due to the $\psi^{*}$ component, and not to $m(\theta, t)$, since the correlation between the filtered SDF and the FF factors is the same as that between the filtered missing component of the SDF and the FF factors.

The above results are perhaps not surprising because the FF factors are known to be quite successful in explaining a large fraction of the cross-sectional variation in the returns of the 25 size and book-to-market-equity-sorted portfolios. However, panels $B$ and $C$ reveal that the filtered SDF strongly correlates with the FF factors independently of the set of test assets used to extract the filtered SDF. When the set of test assets consists of the 10 momentum-sorted portfolios, the correlations vary from 0.51 to 0.55 . For the 10 industry-sorted portfolios, the correlations vary from 0.53 to 0.69 . Column 4 of panels $B$ and $C$ reveals that this high correlation is almost entirely driven by the missing component of the SDF, and not the consumption growth component.

Row 1, Column 1, of each panel shows that, for the CC model, while the filtered SDF correlates strongly with the FF factors, the model-implied SDF has a small correlation coefficient of 0.18 , when the surplus consumption ratio is extracted from consumption data. Row 1, Column 2, shows that the correlation only rises marginally to 0.20 when the state variable is extracted from the market-wide price-dividend ratio. 
Table 4

Correlations with FF3, 1929-2009

Correlation with FF3

\begin{tabular}{lcccc}
\hline & $\left(\ln M_{t}^{m}\right)_{\text {cons }}$ & $\left(\ln M_{t}^{m}\right)_{\text {prices }}$ & $\ln M_{t}^{*}$ & $\ln \psi_{t}^{*}$ \\
\hline A. 6 Fama-French & & & & \\
\hline CC & 0.19 & 0.12 & $0.73 / 0.78$ & $0.72 / 0.77$ \\
MSV & 0.26 & 0.87 & $0.73 / 0.78$ & $0.72 / 0.77$ \\
BY & 0.38 & 0.73 & $0.77 / 0.77$ & $0.68 / 0.72$ \\
PST & 0.35 & - & $0.81 / 0.76$ & $0.65 / 0.67$ \\
B. 10 Momentum & & & & \\
\hline CC & 0.19 & 0.12 & $0.55 / 0.63$ & $0.58 / 0.61$ \\
MSV & 0.26 & 0.87 & $0.55 / 0.62$ & $0.57 / 0.61$ \\
BY & 0.38 & 0.73 & $0.69 / 0.69$ & $0.51 / 0.57$ \\
PST & 0.35 & - & $0.73 / 0.70$ & $0.50 / 0.55$ \\
C. 10 Industry & & & & \\
\hline CC & 0.19 & 0.12 & $0.49 / 0.53$ & $0.49 / 0.53$ \\
MSV & 0.26 & 0.87 & $0.50 / 0.54$ & $0.50 / 0.55$ \\
BY & 0.38 & 0.73 & $0.42 / 0.39$ & $0.38 / 0.42$ \\
PST & 0.35 & - & $0.41 / 0.27$ & $0.34 / 0.37$ \\
\hline
\end{tabular}

The table reports the correlations between the 3 Fama-French factors and (1) the model-implied SDF with state variables extracted from consumption (Column 1) and stock market (Column 2) data, (2) the filtered SDF (Column 3), and (3) the filtered $\psi^{*}$ component of the SDF (Column 4), using annual data for 1929-2009 and a different set of portfolios in each panel. Each cell in Columns 3 and 4 has two entries corresponding to whether the filtered $\psi^{*}$-component and, therefore, the filtered SDF is estimated using Equation 6, reported on the left, or Equation 4 . reported on the right. $C C, M S V, B Y$, and $P S T$ denote, respectively, the models of Campbell and Cochrane 1999), Menzly et al, 2004), Bansal and Yaron 2004), andPiazzesi et al, 2007).

For the MSV model, the correlation between the model-implied SDF and the FF factors is small at 0.21 when the surplus consumption ratio is extracted from consumption data. However, when the state variable is extracted from the price-dividend ratio, the correlation between the model-implied SDF and the FF factors is very high at 0.95 - much higher than the correlation between the filtered SDF and the FF factors for each set of test assets.

Row 3 in each panel shows that for the BY model, the correlation between the model-implied SDF and the FF factors is 0.25 when the state variables are extracted from consumption data. The correlation increases to 0.45 when asset price data are used in the extraction of the model-implied state variables.

Finally, Row 4 in each panel shows that for the PST model, the correlation between the model-implied SDF and the FF factors is very small at 0.07 .

Table 4 reveals that very similar results are obtained at the annual frequency. Tables 3 and 4 demonstrate the soundness of our estimation method: the filtered time series of the SDF and its $\psi^{*}$ component are quite robust, in terms of their correlations with the FF factors, to the choice of the utility curvature parameter $\gamma$, the set of assets, and the data frequency considered. Moreover, our filtered SDF and $\psi^{*}$ are consistently highly correlated with the FF factors independently of the sample frequency and the cross-section of assets used for the estimation (even assets, like the industry and momentum portfolios, that are not well priced by the FF factors). This finding has several important implications. First, it suggests that our estimation approach successfully identifies the unobserved 
pricing kernel, since there is substantial empirical evidence that the FF factors do proxy for asset risk sources. Second, our finding provides a rationalization of the empirical success of the FF factors in pricing asset returns. Finally, although the filtered SDF is highly correlated with the FF factors, the correlation coefficient is substantially smaller than unity, particularly for the industry and momentum portfolios (see, e.g., Table 4), suggesting that the FF factors cannot fully capture all the underlying sources of systematic risk that are important in pricing these assets.

The reason behind the stable correlation results between our filtered SDFs and the three Fama French factors seems to be the fact that, independently of the set of assets used for the filtering, the filtered SDF tends to have a very similar time series behavior. In particular, it shows a clear business-cycle pattern, and significant and sharp reactions to stock market crashes (even if these crashes do not necessarily result in economy wide contractions). This feature of the filtered SDFs is illustrated in Figures 9 (annual frequency) and 10 (quarterly frequency). In each figure we report the business-cycle component (panel A) and the residual component of the filtered $M^{*}$ for the different models 18 At both data frequencies, of from the model considered, both the business-cycle and residual components are extremely similar across the models.

In Table 5 we compare the business-cycle and market crash properties of the filtered SDFs with the model-implied ones. For each model considered, and for both the filtered $\left(M^{*}\right)$ and model-implied $\left(M^{m}\right)$ pricing kernels, the table reports the risk-neutral probabilities of recessions (Column 1), and stock market crashes nonconcomitant with recessions (Column 2), as well as in the first row of each panel, the sample frequency of these events 19 For the modelimplied pricing kernels, we present the probabilities when the state variables are extracted using consumption data as well as using asset price data (in brackets below).

Focusing on quarterly data (panel A), Column 1 shows that the filtered SDFs $\left(M^{*}\right)$ imply a risk-neutral probability of a recession in the $25 \%-26 \%$ range. Comparing this with the model-implied probabilities reveals that, whether the state variables are extracted using consumption or asset market data, all the model-implied pricing kernels deliver a similar risk-neutral probability of recessions that is similar to the one of our filtered SDFs (with the notable exception of the BY pricing kernel that, extracting the state variables using asset

18 The decomposition into a business-cycle and a residual component is obtained by applying the Hodrick and Prescott 1997) filter to the estimated $M^{*}$.

19 To compute the risk-neutral probabilities, note that for any quantity $A_{t}$ and function $f($.$) , we have that$ $\mathbb{E}^{Q}\left[f\left(A_{t}\right)\right]=\int f\left(A_{t}\right) \frac{d Q}{d P} d P=\int f\left(A_{t}\right) \frac{M_{t}}{\bar{M}} d P$. Hence, given an SDF $M_{t}$ (either filtered or model-implied) the risk-neutral expectation can be estimated (assuming ergodicity) using the sample analog $\widehat{\mathbb{E}} \widehat{\left.\left.Q_{\left[f\left(A_{t}\right.\right.}\right)\right]}=$ $\frac{1}{T} \sum_{t}^{T} f\left(A_{t}\right) \frac{M_{t}}{\bar{M}}$. For instance, to estimate the probability of a recession, we replace $f\left(A_{t}\right)$ with an index function that takes value 1 if the economy was in an NBER-designated recession at time $t$ and zero otherwise. See also Remark1 1 in Appendix A.1 
Panel A: Business Cycle components of $\mathbf{M}^{*}$

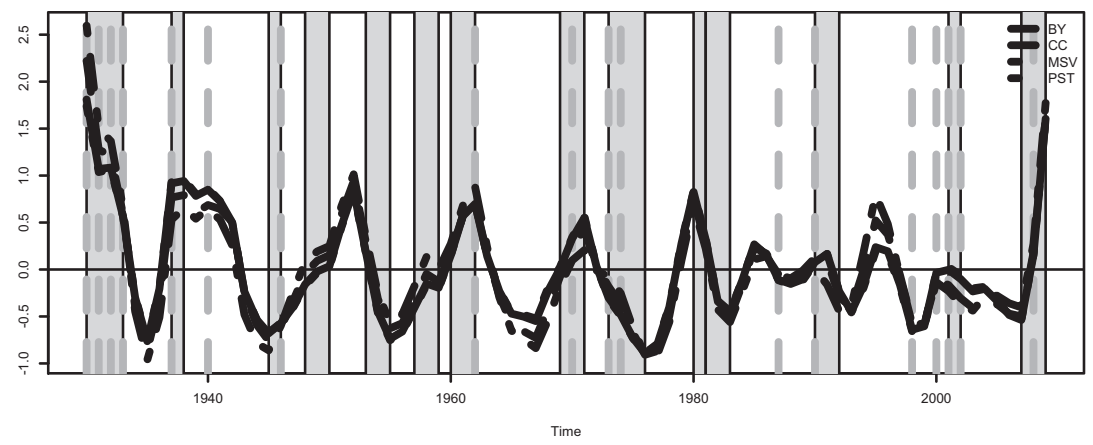

Panel B: Residual components of $\mathbf{M}^{*}$

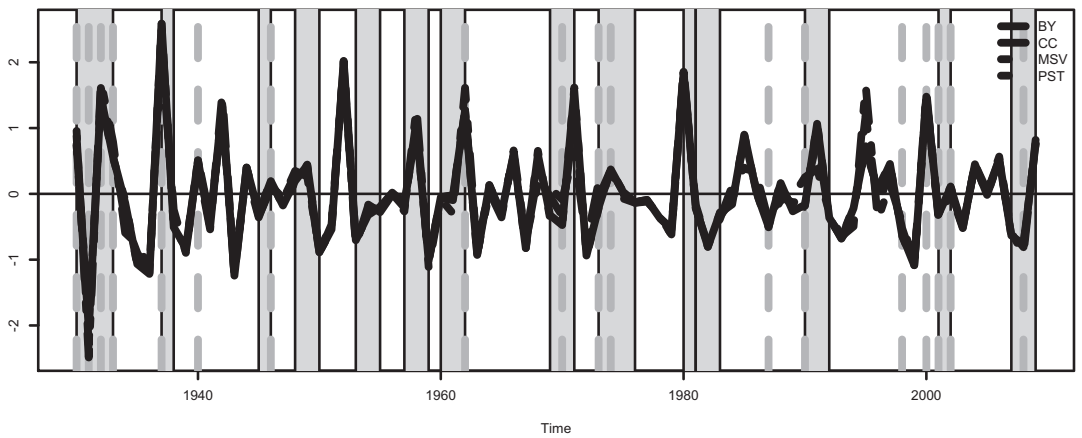

Figure 9

Business-cycle $(A)$ and residual $(B)$ components of the filtered $(\log ) \operatorname{SDF}\left(M_{t}^{*}=\left(\frac{C_{t}}{C_{t-1}}\right)^{-\gamma} \psi_{t}^{*}\right)$ filtered using the relative entropy-minimizing procedure in Equation 6 using annual data over the period 1929-2009 for the different models considered:Bansal and Yaron 2004) (BY).Campbell and Cochrane 1999) (CC). Menzly et al. 2004) (MSV), andPiazzesi et al. 2007) (PST). The difference between the models is driven by the value of the utility curvature parameter $\gamma$ that is set to the authors' original calibrations. The decomposition into a business-cycle and a residual component is obtained by applying the Hodrick and Prescot 1997) filter to the estimated $M^{*}$. The set of test assets used in the filtering consists of the six size and book-to-market-equity-sorted portfolios, ten industry-sorted portfolios, and the ten momentum-sorted portfolios. Shaded areas denote NBER recession years, and vertical dashed lines indicate the major stock market crashes identified by Mishkin and White 2002).

market data, implies a risk-neutral probability of recession of about 55\%). More interestingly, Column 2 shows that the model-implied kernels fail to show the significant and sharp reaction to stock market crashes exhibited by the filtered SDFs: the probabilities of stock market crashes nonconcomitant with recessions implied by the filtered SDFs are between $104 \%$ and $207 \%$ higher than those implied by the model specific kernels when the model-implied state variables are extracted from consumption data and between $44 \%$ and $207 \%$ higher when the state variables are extracted from asset price data. Panel B reports similar findings at the annual frequency, but also shows that MSV and PST imply too 
Panel A: Business Cycle components of $\mathbf{M}^{*}$

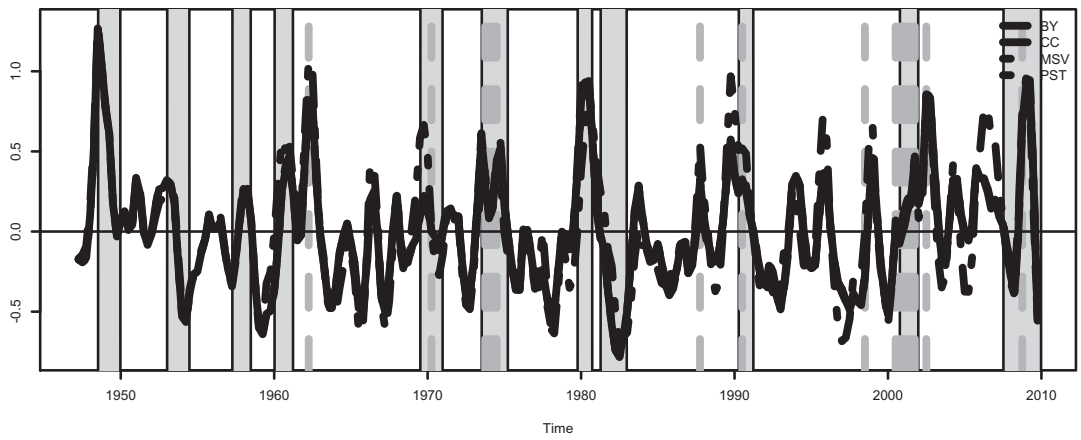

Panel B: Residual components of $\mathbf{M}^{*}$

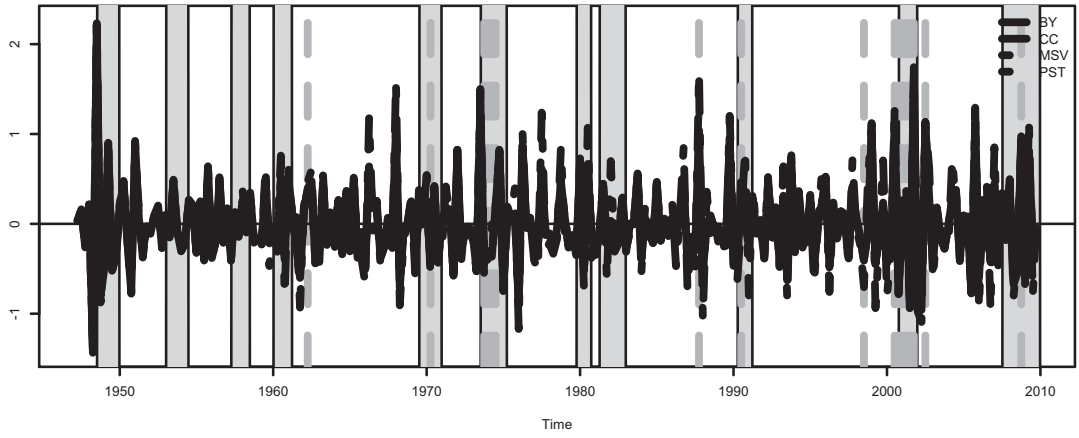

Figure 10

Business-cycle $(A)$ and residual $(B)$ components of the filtered $(\log ) \operatorname{SDF}\left(M_{t}^{*}=\left(\frac{C_{t}}{C_{t-1}}\right)^{-\gamma} \psi_{t}^{*}\right)$ filtered using the relative entropy-minimizing procedure in Equation 6 using quarterly data over the period 1947:Q1-2009:Q4 for the different models considered: Bansal and Yaron 2004) (BY).Campbell and Cochrane 1999) (CC). Menzly et al. 2004) (MSV), andPiazzesi et al. 2007) (PST). The difference between the models is driven by the value of the utility curvature parameter $\gamma$ that is set to the authors' original calibrations. The decomposition into a business-cycle and a residual component is obtained by applying the Hodrick and Prescot 1997) filter to the estimated $M^{*}$. The set of test assets used in the filtering consists of the six size and book-to-market-equity-sorted portfolios, ten industry-sorted portfolios, and the ten momentum-sorted portfolios. Shaded areas denote NBER recession years, and vertical dashed lines indicate the major stock market crashes identified by Mishkin and White 2002).

low of probablities for recessions and BY - only when extraxted from asset prices - implies a very high probability of market crash 20

Overall, the above results suggest that the explanatory power of these models for asset pricing would be improved by augmenting the pricing kernels with a component that exhibits sharp reactions to market crashes that are not perfectly correlated with the business cycle.

20 Note that, at the annual frequency, a year is designated as a recession year if at least one of its quarters is in an NBER recession period. 
Table 5

Recession and market crash probabilities of $M^{m}$ and $M^{*}$

\begin{tabular}{lccc} 
& & $\begin{array}{c}\text { Recession } \\
\text { Recession probability }\end{array}$ & $\begin{array}{c}\text { Market crash without } \\
\text { recession probability }\end{array}$ \\
\hline A. Quarterly Data, 1947:Q1-2009:Q4 & & 0.024 \\
Sample & $M^{m}$ & .022 & $0.024[0.025]$ \\
CC & $M^{*}$ & $0.25[0.24]$ & $0.054 / 0.059$ \\
$B Y$ & $M^{m}$ & $0.25 / 0.25$ & $0.024[0.034]$ \\
& $M^{*}$ & $0.22[0.55]$ & $0.049 / 0.057$ \\
MSV & $M^{m}$ & $0.26 / 0.26$ & $0.024[0.028]$ \\
& $M^{*}$ & $0.22[0.22]$ & $0.055 / 0.059$ \\
PST & $M^{m}$ & $0.25 / 0.25$ & 0.028 \\
& $M^{*}$ & 0.21 & $0.086 / 0.065$ \\
\hline B. Annual Data, 1929-2009 & $0.25 / 0.25$ & 0.088 \\
\hline Sample & & & $0.055[0.068]$ \\
CC & $M^{m}$ & 0.375 & $0.092 / 0.119$ \\
BY & $M^{*}$ & $0.61[0.49]$ & $0.083[0.227]$ \\
& $M^{m}$ & $0.58 / 0.56$ & $0.085 / 0.097$ \\
MSV & $M^{*}$ & $0.41[0.59]$ & $0.086[0.098]$ \\
& $M^{m}$ & $0.59 / 0.59$ & $0.094 / 0.122$ \\
PST & $M^{*}$ & $0.38[0.39]$ & 0.067 \\
& $M^{m}$ & $0.57 / 0.56$ & $0.103 / 0.093$ \\
\hline
\end{tabular}

The table reports the risk-neutral probability of recessions (Column 1) and stock market crashes nonconcomitant with recessions (Column 2) implied by the model $\left(M^{m}\right)$ and filtered $\left(M^{*}\right)$ SDFs at quarterly (panel A) and annual (panel B) frequencies. Each cell in the rows corresponding to the model SDF has two entries corresponding to whether the models' state variables are extracted from consumption data, reported on the left, or from asset market data, reported on the right. Each cell in the rows corresponding to the filtered SDF has two entries corresponding to whether the filtered $\psi^{*}$-component and, therefore, the filtered SDF is estimated using Equation 6 , reported on the left, or Equation 4, reported on the right.

3.2.2 Entropy Bounds Analysis. Our second approach to assess the empirical plausibility of the asset pricing models considered relies on the entropy bounds derived in Section 1.1 For each model we compute the minimum values of the power coefficient, $\gamma$, at which the model-implied SDF satisfies the $H J, Q, M$, and $\Psi$ bounds. We also compute $95 \%$ confidence bands via bootstrap. Table 6 reports the results at the quarterly frequency. Panels A and B report results when the state variables needed to construct the time series of the model-implied SDF and its components are extracted from consumption (panel A) and asset market data (panel B). Consider first the results for the $H J, Q 1, M 1$, and $\Psi 1$ bounds. The first row in each panel presents the bounds for the CC model. Panel A shows that when the surplus consumption ratio is extracted from consumption data, the minimum values of $\gamma$ at which the pricing kernel satisfies the $H J, Q 1$, $M 1$, and $\Psi 1$ bounds are 10.2, 16.1, 16.4, and 23.2, respectively. Therefore, as suggested by the theoretical predictions, the $Q$-bound is tighter than the $H J$ bound, and the $M$-bound is tighter than the $Q$-bound. Note that in this model, the curvature of the utility function is $\frac{\gamma}{S_{t}}$, where $S_{t}$ is the surplus consumption ratio, and this ratio is almost identical to the coefficient of relative-risk aversion (see, e.g., the discussion in Campbell and Cochrane 1999)). For $\gamma=2$, the calibrated value in CC, the curvature varies over $[19.7, \infty)$. Panel A reveals that 
Table 6

Bounds for RRA, quarterly data, 1947:Q1-2009:Q4

\begin{tabular}{|c|c|c|c|c|}
\hline & $H J$-bound & $Q 1 / Q 2$-bounds & $M 1 / M 2$-bounds & $\Psi 1 / \Psi 2$-bounds \\
\hline \multicolumn{5}{|c|}{ A. State variables extracted from consumption } \\
\hline$\overline{C C}$ & 10.2 & $\begin{array}{c}16.1 / 15.7 \\
{[16.0,38.0][14.4,34.8]}\end{array}$ & $\begin{array}{c}16.4 / 16.0 \\
{[16.0,38.0][14.6,36.8]}\end{array}$ & $\begin{array}{c}23.2 / 23.9 \\
{[23.0,>100][21.2,>100]}\end{array}$ \\
\hline$M S V$ & 32.6 & $\begin{array}{c}40.8 / 40.4 \\
{[38.0,62.0][38.0,59.0]}\end{array}$ & $\begin{array}{c}43.4 / 43.5 \\
{[40.0,64.0][40.0,64.0]}\end{array}$ & $\begin{array}{c}61.3 / 62.8 \\
{[59,113][59.0,>100]}\end{array}$ \\
\hline$B Y$ & $>100$ & $\begin{array}{c}>100 />100 \\
{[>100,>100][>100,>100]}\end{array}$ & $\begin{array}{c}>100 />100 \\
{[>100,>100][>100,>100]}\end{array}$ & $\begin{array}{c}>100 />100 \\
{[>100,>100][>100,>100]}\end{array}$ \\
\hline$P S T$ & 73.8 & $\begin{array}{c}99.0 / 92.6 \\
{[96.0,172.0][88.0,161.0]}\end{array}$ & $\begin{array}{c}111.1 / 102.2 \\
{[102.0,183.0][93.0,172,1]}\end{array}$ & $\begin{array}{c}96.2 / 90.5 \\
{[94.0,187.0][86.0,176.0]}\end{array}$ \\
\hline \multicolumn{5}{|c|}{ B. State variables extracted from asset prices } \\
\hline$\overline{C C}$ & 19 & $\begin{array}{c}43 / 46 \\
{[43.0,50.0][46.0,49.0]}\end{array}$ & $\begin{array}{c}46 / 46 \\
{[46.0,50.0][46.0,49.0]}\end{array}$ & $\begin{array}{c}47 / 48 \\
{[47.0,51.0][48.0,50.0]}\end{array}$ \\
\hline$M S V$ & 73.3 & $\begin{array}{c}90.3 / 90.0 \\
{[92.0,>100][89.5,>100]}\end{array}$ & $\begin{array}{c}>100 />100 \\
{[>100,>100][>100,>100]}\end{array}$ & $\begin{array}{c}>100 />100 \\
{[>100,>100][>100,>100]}\end{array}$ \\
\hline$B Y$ & 4.0 & $\begin{array}{c}5 / 5 \\
{[5.0,6.0][5.0,6.0]}\end{array}$ & $\begin{array}{c}5 / 5 \\
{[5.0,6.0][5.0,6.0]}\end{array}$ & $\begin{array}{c}5 / 5 \\
{[5.0,6.0][5.0,6.0]}\end{array}$ \\
\hline
\end{tabular}

The table reports the minimum values of the utility curvature parameter $\gamma$ at which the model-implied SDF satisfies the HJ (Column 1), Q (Column 2), M (Column 3), and $\Psi$ (Column 4) bounds using quarterly data for 1947:Q1-2009:Q4. The bootstrapped 95\% confidence intervals are reported in brackets below. Columns 2-4 has two entries in each cell that correspond to whether the filtered $\psi^{*}$-component of the SDF and, therefore, the filtered SDF are estimated using equation 6, reported on the left, or Equation 4, reported on the right. Panels A and B present results when the models' state variables are extracted from consumption data and asset market data, respectively. $C C, M S V, B Y$, and $P S T$ denote, respectively, the models of Campbell and Cochrane 1999), Menzly et al, 2004, Bansal and Yaron 2004, and Piazzesi et al, 2007).

the $Q$-bound is satisfied for $\gamma \geqslant 16.1$, implying that the curvature varies over $[56.6, \infty)$, the $M$-bound is satisfied for $\gamma \geqslant 16.4$, implying that the curvature varies over $[57.2, \infty)$, and the $\Psi$-bound is satisfied for $\gamma \geqslant 23.2$, implying that the curvature varies over $[68.5, \infty)$. A similar ordering of the bounds is obtained when the surplus consumption ratio is extracted from the market-wide pricedividend ratio in panel $\mathrm{B}$, except that, in this case, even higher values of risk aversion are needed to satisfy the bounds. Also, very similar results are obtained for the $Q 2, M 2$, and $\Psi 2$ bounds, stressing the robustness of our approach.

The second row in each panel presents the bounds for the MSV model. When the surplus consumption ratio is extracted from consumption data, the $H J, Q 1$, $M 1$, and $\Psi 1$ bounds are satisfied for a minimum value of $\gamma=32.6,40.8,43.4$, and 61.3 , respectively. Very similar results are obtained for the $Q 2, M 2$, and $\Psi 2$ bounds. Therefore, this model requires much higher values of risk aversion than $\mathrm{CC}$ to be consistent with observed asset returns. Note, however, that for both models and both procedures used to extract the model-implied SDFs, the risk-aversion coefficients at which the models satisfy the bounds are very high.

The third row in each panel presents the bounds for the BY model. Panel A shows that when the model-implied state variables are extracted from consumption data, the model-implied pricing kernel fails to satisfy the $H J$, $Q, M$, and $\Psi$ bounds for any value of the risk-aversion parameter smaller than 100. On the contrary, when the model-implied state variables are extracted from 
asset market data (panel B), the $H J$ bound is satisfied for a minimum value of $\gamma=4.0$, while the $Q 1, M 1$, and $\Psi 1$ bounds are all satisfied by a relative riskaversion as small as 5. Similar results are obtained for the $Q 2, M 2$, and $\Psi 2$ bounds. Therefore, the results reveal that the empirical performance of the BY framework crucially depends on how the latent state variables are extracted from the data.

Finally, the fourth row of panel A presents the bounds for the PST model. Note that, in this model, the SDF is a function of observable data alone, hence there is no need to extract any state variable from asset market data. Therefore, we do not have a fourth row in panel B. The model satisfies the $H J, Q 1(Q 2)$, $M 1(M 2)$, and $\Psi 1(\Psi 2)$ bounds for minimum values of $\gamma=73.8,99.0(92.6)$, 111.1(102.2), and 96.2(90.5), respectively. Therefore, this model requires very high levels of risk-aversion to be consistent with observed asset returns.

Overall, Table 6 demonstrates that, in line with the theoretical underpinnings of the various bounds, the $Q$-bound is generally tighter than the $H J$-bound because it naturally exploits the restriction that the SDF is a strictly positive random variable. The $M$-bound is tighter than the $Q$-bound because it formally takes into account the ability of the SDF to price assets and the dependency of the pricing kernel on consumption. Furthermore, the results suggest that all the models considered require very high levels of risk-aversion to satisfy the bounds, with the only exception being the long-run risks model of BY (but only when the model state variables are extracted from asset price data).

Table 7 reports analogous bounds as in Table 6 at the annual frequency. The table shows that, at this frequency, all the bounds tend to be satisfied with smaller values of the utility curvature parameter, suggesting that the models considered can more easily rationalize asset pricing dynamics at the annual, rather than quarterly, frequency. However, once again in line with the theoretical predictions, the $Q$-bound is tighter than the $H J$-bound, and the $M$-bound is tighter than the $Q$-bound.

Note that the above bound results have tight confidence bands and are much more consistent, in evaluating the plausibility of a given model across different procedures used to extract the model-implied SDF and its components, than the cross-sectional $R^{2}$ measures reported in Tables 1 and 2 that wildly vary for the same model and have very wide confidence intervals.

Note that the results in Tables 6 and 7 are obtained by allowing only the utility curvature parameter, $\gamma$, to vary while holding constant all the other model parameters at the authors' calibrated values. Note that most consumption based asset pricing models, including the ones considered in this paper, are highly parameterized. Since the state variables are not directly observed in many of the models, the parameters governing their dynamics are typically chosen to match some moments of the data. Consequently, the properties of the SDF are quite sensitive to not only $\gamma$ but also the values of all the other parameters. Therefore, we also compute the minimum values of the power coefficient, $\gamma$, 
Table 7

Bounds for RRA, annual data, 1929-2009

\begin{tabular}{|c|c|c|c|c|}
\hline & $J J$-bound & $Q 1 / Q 2$-bounds & $M 1 / M 2$-bounds & $\Psi 1 / \Psi 2$-bounds \\
\hline \multicolumn{5}{|c|}{ A. State variables extracted from consumption } \\
\hline$\overline{C C}$ & 0.7 & $\begin{array}{c}5.1 / 2.7 \\
{[4.0,41.0][3.0,8.0]}\end{array}$ & $\begin{array}{c}5.2 / 2.7 \\
{[4.0,41.0][3.0,8.0]}\end{array}$ & $\begin{array}{c}7.6 / 3.6 \\
{[5.0,>100][4.0,23.2]}\end{array}$ \\
\hline$M S V$ & 17 & $\begin{array}{c}28.7 / 24.4 \\
{[19.0,53.3][23.7,35.0]}\end{array}$ & $\begin{array}{c}30.3 / 26.6 \\
{[20.0,53.3][24.7,35.4]}\end{array}$ & $\begin{array}{c}>100 / 76.5 \\
{[>100,>100][81.0,>100]}\end{array}$ \\
\hline$B Y$ & 50 & $\begin{array}{c}53 / 71 \\
{[22.0,71.0][69.7,>80]}\end{array}$ & $\begin{array}{c}60 />80 \\
{[24.0,72.0][>80,>80]}\end{array}$ & $\begin{array}{c}55 />80 \\
{[49.0,>80][2.0,>80]}\end{array}$ \\
\hline PST & 17.1 & $\begin{array}{c}28.6 / 24.1 \\
{[19.0,51.7][23.0,35.4]}\end{array}$ & $\begin{array}{c}31.4 / 27.0 \\
{[20.0,51.3][24.0,35.4]}\end{array}$ & $\begin{array}{c}22.0 / 18.6 \\
{[14.0,42.7][19.7,29.0]}\end{array}$ \\
\hline \multicolumn{5}{|c|}{ B. State variables extracted from asset prices } \\
\hline$C C$ & 4 & $\begin{array}{c}7 / 6 \\
{[4.0,12.0][6.0,9.0]}\end{array}$ & $\begin{array}{c}7 / 6 \\
{[4.0,12.0][6.0,9.0]}\end{array}$ & $\begin{array}{c}8 / 7 \\
{[4.0,14.0][7.0,11.0]}\end{array}$ \\
\hline$M S V$ & 23.7 & $\begin{array}{c}39.1 / 33.4 \\
{[22.0,69.5][29.5,45.0]}\end{array}$ & $\begin{array}{c}42.2 / 37.0 \\
{[26.0,69.5][30.5,45.0]}\end{array}$ & $\begin{array}{c}>100 />100 \\
{[>100,>100][>100,>100]}\end{array}$ \\
\hline$B Y$ & 5 & $\begin{array}{c}6 / 6 \\
{[5.0,6.0][2.0,7.0]}\end{array}$ & $\begin{array}{c}6 / 6 \\
{[5.0,6.0][2.0,6.0]}\end{array}$ & $\begin{array}{c}6 / 6 \\
{[5.0,6.0][2.0,6.0]}\end{array}$ \\
\hline
\end{tabular}

The table reports the minimum values of the utility curvature parameter $\gamma$ at which the model-implied SDF satisfies the HJ (Column 1), Q (Column 2), M (Column 3), and $\Psi$ (Column 4) bounds using annual data for 1929-2009. The bootstrapped 95\% confidence intervals are reported in brackets. Columns 2-4 has two entries in each cell that correspond to whether the filtered $\psi^{*}$-component of the SDF and, therefore, the filtered SDF are estimated using equation 6, reported on the left, or Equation 4, reported on the right. Panels A and B present results when the models' state variables are extracted from consumption data and asset market data, respectively. $C C, M S V, B Y$, and PST denote, respectively, the models of Campbell and Cochrane 1999, Menzly et al, 2004, Bansal and Yaron 2004, and Piazzesi et al, 2007).

at which the model-implied SDFs satisfy the $H J, Q, M$, and $\Psi$ bounds while allowing the remaining model parameters to simultaneously vary over two standard-error intervals around their calibrated values. The results, reported in Table A2 of Appendix A.7.1 remain qualitatively unchanged. In particular, for each model, the $H J, Q, M$, and $\Psi$ bounds are satisfied for smaller values of $\gamma$ when the other parameters are allowed to vary simultaneously compared to Tables 6 and 7 where the other parameters are held fixed. However, as in the latter tables, the $C C, M S V$, and $P S T$ models still require much larger values of risk-aversion to satisfy the bounds compared to the authors' calibrated values at the quarterly frequency.

Also note that we have used excess returns (in excess of the risk-free rate) on a broad cross section of risky assets to extract SDF and obtain entropy bounds on the SDF and its components. However, it is well known that the level of the risk-free asset constrains models quite dramatically. Therefore, in order to check the robustness of our results, we repeat the empirical exercise using as test assets the gross returns (instead of excess returns) on the same assets considered so far plus the risk-free asset. The methodology needs to be slightly modified in this case and is described in Appendix A.7.2 The results, reported in Table A3 of Appendix A.7.2 show that the inclusion of the risk-free rate as an additional asset leaves the $H J, Q, M$, and $\Psi$ bounds on the SDF and its components very similar to those obtained in Tables 6 and 7 without the risk-free rate, for all the models considered. 
Table 8

Relative entropy of SDF and SDF components

\begin{tabular}{|c|c|c|c|}
\hline & $\operatorname{KLIC}\left(M_{t}^{m}\right)$ & $\operatorname{KLIC}\left(\psi_{t}^{\text {resid }}\right)$ & $\frac{K \operatorname{LIC}\left(\psi_{t}^{\text {resid }}\right)}{K \operatorname{LIC}\left(M_{t}^{m} \psi_{t}^{\text {resid }}\right)}$ \\
\hline \multicolumn{4}{|c|}{ A. Quarterly, 1947:Q1-2009:Q4 } \\
\hline$\overline{C C}$ & $\begin{array}{c}0.035 / 0.037 \\
{[0.018][0.019]}\end{array}$ & $\begin{array}{c}0.26 / 0.32 \\
{[0.30][0.33]}\end{array}$ & $\begin{array}{c}0.772 / 0.786 \\
{[0.909][0.859]}\end{array}$ \\
\hline$M S V$ & $\begin{array}{c}0.0002 / 0.0002 \\
{[0.004][0.004]}\end{array}$ & $\begin{array}{c}0.31 / 0.36 \\
{[0.30][0.35]}\end{array}$ & $\begin{array}{c}0.992 / 0.996 \\
{[0.950][0.953]}\end{array}$ \\
\hline$B Y$ & $\begin{array}{c}0.003 / 0.003 \\
{[1.69][1.70]}\end{array}$ & $\begin{array}{c}0.30 / 0.35 \\
{[0.59][0.39]}\end{array}$ & $\begin{array}{c}0.957 / 0.971 \\
{[0.647][0.448]}\end{array}$ \\
\hline PST & $0.008 / 0.008$ & $0.39 / 0.39$ & $1.01 / 0.989$ \\
\hline \multicolumn{4}{|c|}{ B. Annual, 1929-2009 } \\
\hline$\overline{C C}$ & $\begin{array}{c}0.379 / 0.660 \\
{[0.164][0.169]}\end{array}$ & $\begin{array}{c}0.66 / 0.69 \\
{[0.76][0.73]}\end{array}$ & $\begin{array}{c}0.688 / 0.676 \\
{[0.815][0.767]}\end{array}$ \\
\hline$M S V$ & $\begin{array}{c}0.001 / 0.001 \\
{[0.023][0.023]}\end{array}$ & $\begin{array}{c}0.85 / 0.85 \\
{[0.85][0.81]}\end{array}$ & $\begin{array}{c}0.972 / 0.974 \\
{[0.973][0.906]}\end{array}$ \\
\hline$B Y$ & $\begin{array}{l}0.023 / 0.022 \\
{[2.66][1.75]}\end{array}$ & $\begin{array}{c}0.82 / 0.84 \\
{[2.33][1.02]}\end{array}$ & $\begin{array}{r}0.932 / 0.959 \\
{[1.44][0.712]}\end{array}$ \\
\hline$P S T$ & $0.19 / 0.27$ & $0.96 / 0.91$ & $1.06 / 0.996$ \\
\hline
\end{tabular}

The table reports the KLIC of the model-implied SDF (Column 1), the KLIC of the residual psi (Column 2), and the ratio of the KLIC of the residual psi and the KLIC of the product of the model-implied SDF and the residual psi (Column 3) at the quarterly (panel A) and annual (panel B) frequencies. Each cell has four entries that correspond to whether the models' state variables are extracted from consumption data, reported at the top, or from asset market data, reported at the bottom, and to whether the KLIC between measure A and the physical measure $\mathrm{P}$ is computed as $\mathrm{D}(\mathrm{P} \| \mathrm{A})$, reported on the left, or as $\mathrm{D}(\mathrm{A} \| \mathrm{P})$, reported on the right. $C C, M S V, B Y$, and PST denote, respectively, the models of Campbell and Cochrane 1999). Menzly et al. 2004), Bansal and Yaron 2004), and Piazzesi et all 2007).

3.2.3 What Are The Consumption-Based Models Missing?. As shown in Section 1.1.1 modeling the SDF as fully observable, that is, setting $m(\theta, t)=M_{t}^{m}$, where $M_{t}^{m}$ is the entire pricing kernel of the model under consideration (given in Equations 210, 24], and (25), we can extract a residual $\psi^{\text {resid }}$ component such that $M_{t}^{*}:=M_{t}^{m} \times \psi_{t}^{\text {resid }}$ correctly prices assets. The $\psi^{\text {resid }}$ component can once again be estimated using the relative entropy minimization procedures in Equations (4) and (6) replacing $m$ with $M^{m}$. The $\psi^{\text {resid }}$ multiplicative adjustment of the pricing kernel: (1) still has an maximum likelihood interpretation, (2) adds the minimum amount of information needed for $M^{*}$ to be able to price assets correctly, and (3) most importantly, as the second Hansen-Jagannathan distance, it provides a useful diagnostic for detecting what the pricing kernels are missing to be consistent with observed asset returns.

We first examine the relative importance of the two components of $M^{*}, M^{m}$ and $\psi^{\text {resid }}$, in pricing a broad cross-section of assets. We do this by computing the contribution of each component to the overall entropy of the pricing kernel. The results are reported in Table 8 Columns 1 and 2 present the relative entropy, or KLIC, of the model-implied SDF, $M_{t}^{m}$, and the residual component, $\psi_{t}^{\text {resid }}$, respectively. Column 3 reports the KLIC of $\psi_{t}^{\text {resid }}$ as a fraction of the KLIC of the overall filtered kernel $M_{t}^{m} \times \psi_{t}^{\text {resid }}$. 
Each row of Column 1 reports the KLIC, or relative entropy, of $M_{t}^{m}$. There are four numbers for this quantity since there are two possible ways of computing the KLIC (as $D\left(P \| M^{m}\right)$, reported on the left, and $D\left(M^{m} \| P\right)$, reported on the right), and two possible ways of extracting the models' state variables (from consumption, top numbers, and from asset market data, bottom numbers in brackets). Similarly, four numbers with the same ordering are reported in the remaining two columns. First, consider panel A, that presents results obtained at the quarterly frequency. Columns 1 and 2 show that, for the CC model, the relative entropy of $\psi^{\text {resid }}$ is an order of magnitude bigger than that of $M^{m}$, regardless of whether $\psi_{t}^{\text {resid }}$ is estimated using Equation (4) or 6), or whether $M_{t}^{m}$ is obtained by extracting the state variable from consumption or asset market data. This point is further highlighted in Column 3 that shows that the KLIC of $\psi_{t}^{\text {resid }}$ accounts for a lions share of the KLIC of the overall kernel: $77.2 \%-78.6 \%$ when the model-implied state variable is extracted from consumption data and $85.9 \%-90.9 \%$ when it is extracted from asset price data. Very similar results are obtained for the MSV, BY, and PST models in Rows 2-4, and also at the annual frequency in panel B. Overall, the results suggest that, for each model considered, most of the ability of the kernel to price assets comes from the residual component and very little from the model-implied component, that is all the pricing kernels under consideration seem to miss a substantial share of the information needed to price correctly the observed asset returns.

In order to assess whether these models are missing similar features of the data, Table 9 reports the correlations between the $\psi^{\text {resid }}$ of different models at the quarterly (panel A) and annual (panel B) frequencies. As in the previous table, for all the entries we have four number given by the two ways of computing relative entropy (left and right numbers corresponding to Equations (4) and (6) and the two ways of extracting the models' state variables (from consumption in the top numbers and from asset prices for the numbers below in brackets). Panel A shows that, when the models' state variables are extracted from consumption data, the correlations between the residual $\psi$ 's are extremely high, varying from 0.85 (between CC and PST) to (almost) 1.0 (between MSV and BY) when the $\psi^{\text {resid }}$ component is estimated using Equation (6). When the $\psi^{\text {resid }}$ component is estimated using Equation (4), the correlations are very similar, varying from 0.93 to (almost) 1.0. When the models' state variables are extracted from asset prices the correlations among the various $\psi^{\text {resid }}$ are almost unchanged with one important exception: in this case the correlation between the residual component of the BY model and all other models becomes much smaller ranging from 0.1 to 0.41 . This implies that the BY pricing kernel changes a lot depending on whether its state variables are extracted from market or consumption data. Similar results are obtained at the annual frequency in panel B, although the correlations are generally smaller at this frequency 21

21 Note that the estimates at the annual frequency are inherently more imprecise, due to the small available sample size, than those at the quarterly frequency. 
Table 9

Correlation between residual $\psi$ 's

\begin{tabular}{|c|c|c|c|c|}
\hline & $C C$ & $M S V$ & $B Y$ & PST \\
\hline \multicolumn{5}{|c|}{ A. Quarterly, 1947:Q1-2009:Q4 } \\
\hline$\overline{C C}$ & $\begin{array}{c}1.0 \\
{[0.96][0.95]}\end{array}$ & $\begin{array}{c}0.96 / 0.93 \\
{[0.32][0.10]}\end{array}$ & $\begin{array}{c}0.97 / 0.96 \\
{[0.93][0.94]}\end{array}$ & $0.85 / 0.93$ \\
\hline$M S V$ & - & 1.0 & $\begin{array}{l}1.0 / 1.0 \\
{[0.41][0.20]}\end{array}$ & $\begin{array}{c}0.91 / 0.97 \\
{[0.89][0.94]}\end{array}$ \\
\hline$B Y$ & - & - & 1.0 & $\begin{array}{c}0.91 / 0.97 \\
{[0.26][.10]}\end{array}$ \\
\hline$P S T$ & - & - & - & 1.0 \\
\hline \multicolumn{5}{|c|}{ B. Annual, 1929:2009 } \\
\hline$\overline{C C}$ & 1.0 & $\begin{array}{c}0.87 / 0.66 \\
{[0.91][0.78]}\end{array}$ & $\begin{array}{c}0.88 / 0.77 \\
{[0.40][0.22]}\end{array}$ & $\begin{array}{c}0.80 / 0.51 \\
{[0.83][0.53]}\end{array}$ \\
\hline$M S V$ & - & 1.0 & $\begin{array}{c}0.99 / 0.95 \\
{[0.52][0.27]}\end{array}$ & $\begin{array}{c}0.92 / 0.71 \\
{[0.89][0.62]}\end{array}$ \\
\hline$B Y$ & - & - & 1.0 & $\begin{array}{c}0.88 / 0.62 \\
{[0.38][-0.03]}\end{array}$ \\
\hline PST & - & - & - & 1.0 \\
\hline
\end{tabular}

The table reports the correlations between the residual $\psi$ 's of the different asset pricing models using quarterly data for 1947:Q1-2009:Q4 (panel A) and annual data for 1929-2009 (panel B). Each cell has four entries that correspond to whether the models' state variables are extracted from consumption data, reported at the top, or from asset market data, reported at the bottom, and to whether the residual psi is estimated using Equation 6, reported on the left, or using equation 4, reported on the right. $C C, M S V, B Y$, and PST denote, respectively, the models of Campbell and Cochran 1999, Menzly et al, 2004, Bansal and Yaron 2004, and Piazzesi et al. 2007.

Figure 11 plots the time series of the residual $\psi$ 's for the four models at the quarterly (panel A) and annual (panel B) frequencies, with state variables extracted from consumption data and $\psi^{\text {resid }}$ estimated using Equation (6. The results suggest that these models are all missing a very similar component that would improve their ability to explain asset return dynamics. In particular, all the $\psi^{\text {resid }}$ have a clear business-cycle pattern, but also show significant and sharp reactions to financial market crashes that do not result in economy wide contractions.

To further illustrate this point, Table 10 reports the changes in the modelimplied risk-neutral probabilities need to rationalize stock returns according to $\psi^{\text {resid }}$, that is, the percentage change caused by replacing $M^{m}$ with $M^{m} \times \psi^{\text {resid }}$. As before, we have four entries per model since we compute probabilities when state variables are extracted using consumption data as well as using asset price data (in brackets below), and two minimum entropy methods (left and right numbers). Focusing on quarterly data in panel A, three patterns emerge. First (Column 1), $\psi^{\text {resid }}$ implies a relatively small increase in the riskneutral probability of recessions, suggesting that the models considered tend to adequately capture business-cycle risk at this frequency (with the exception of BY, when the sate variables are extracted from asset prices, that seems to imply too much recession risk). Second (Column 2), all the models seem to imply a too low risk-neutral probability of market crash that is $\psi^{\text {resid }}$ increases this quantity by about $53 \%-98 \%$ (with again the exception of BY that seems to imply 
Panel A

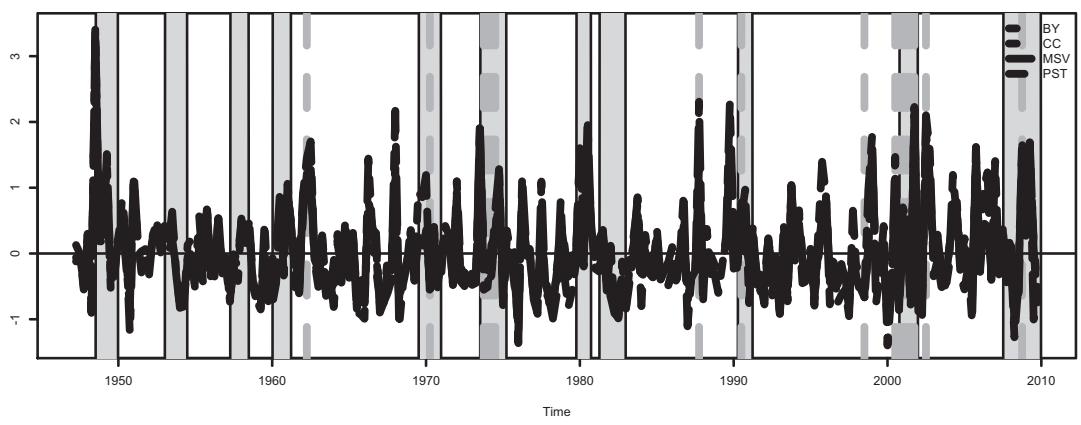

Panel B

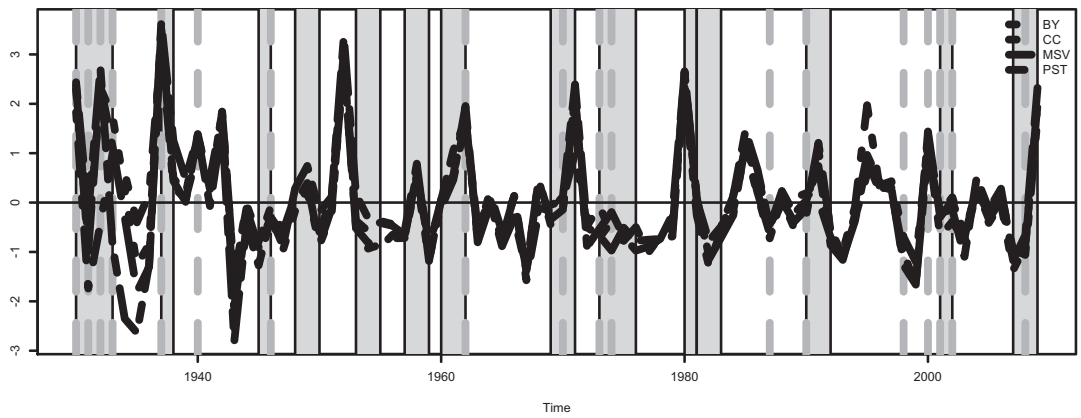

Figure 11

The (log) residual $\psi$ components, $\ln \left(\psi_{t}^{\text {resid }}\right)$, of the SDFs $\left(M_{t}^{*}=M_{t}^{m} \psi_{t}^{\text {resid }}\right)$ filtered using the relative entropyminimizing procedure in Equation 6 using quarterly data for 1947:Q1-2009:Q4 $(A)$ and annual data over the period 1929-2009 $(B)$ for the different models considered: Bansal and Yaron 2004) (BY), Campbell and Cochrane 1999) (CC), Menzly et all 2004) (MSV), and Piazzesi et al. 2007) (PST). Shaded areas denote NBER recession years, and vertical dashed lines indicate the major stock market crashes identified by Mishkin and White 2002).

too much crash risk). Third (Column 3), all the models imply a much too low probability of market crashes not concomitant with recessions: $\psi^{\text {resid }}$ increases the risk-neutral likelihood of these events by about 72\%-232\%. Panel B shows a similar patter, albeit the probability of market crashes without recessions are harder to identify at this frequency. Overall, Table 10 suggests that the models do not seem to price correctly market crash risk, especially market crashes that do not lead to large real economic contractions.

To summarize, the results in this section suggest that the consumption based asset pricing models we have considered would benefit from being augmented with a component that exhibits significant reactions to financial market crashes, in particular crashes that do not result in macroeconomic contractions. Moreover, not only the standard C-CAPM with power utility, but also most of the more recent models that have been proposed in the literature, seem to be missing this component. 
Table 10

Percentage change in risk-neutral probabilities due to residual $\psi$ 's

\begin{tabular}{|c|c|c|c|}
\hline & $\begin{array}{l}\text { Recession } \\
\text { probability }\end{array}$ & $\begin{array}{l}\text { Market crash } \\
\text { probability }\end{array}$ & $\begin{array}{l}\text { Market crash without } \\
\text { recession probability }\end{array}$ \\
\hline \multicolumn{4}{|c|}{ A. Quarterly Data, 1947:Q1-2009:Q4 } \\
\hline \multirow[t]{2}{*}{$\overline{C C}$} & $10 / 11$ & $60 / 59$ & $72 / 105$ \\
\hline & {$[9 / 14]$} & [78/78] & {$[133 / 144]$} \\
\hline \multirow[t]{2}{*}{$B Y$} & $14 / 15$ & $69 / 68$ & $107 / 136$ \\
\hline & {$[-65 /-67]$} & {$[-31 /-32][84 / 144]$} & \\
\hline \multirow[t]{2}{*}{$M S V$} & $15 / 15$ & $78 / 74$ & $124 / 144$ \\
\hline & {$[12 / 11]$} & {$[53 / 53]$} & [93/126] \\
\hline PST & $17 / 20$ & $98 / 75$ & $232 / 148$ \\
\hline \multicolumn{4}{|c|}{ B. Annual Data, 1929-2009 } \\
\hline \multirow[t]{2}{*}{$\overline{C C}$} & $-1 /-1$ & $-2 / 1$ & $10 / 36$ \\
\hline & [21/17] & [73/85] & [11/81] \\
\hline \multirow[t]{2}{*}{$B Y$} & $42 / 37$ & $84 / 86$ & $-2 / 37$ \\
\hline & {$[-22 /-8]$} & {$[-45 / 43]$} & {$[5 /-24]$} \\
\hline \multirow[t]{2}{*}{$M S V$} & $50 / 46$ & $92 / 92$ & $7 / 39$ \\
\hline & [43/39] & [61/63] & {$[3 / 33]$} \\
\hline$P S T$ & $58 / 57$ & $64 / 71$ & $-3 / 69$ \\
\hline
\end{tabular}

The table reports the percentage changes in risk-neutral probabilities generated by the the residual $\psi$ component. recession probabilities. Columns 1 to 3 focus, respectively, on recession, market, and market crash without recession, probabilities. Each cell has four entries that correspond to whether the models' state variables are extracted from consumption data, reported at the top, or from asset market data, reported at the bottom, and to whether the residual psi is estimated using Equation 6, reported on the left, or using Equation 4, reported on the right. CC, MSV, BY and PST denote, respectively, the models of Campbell and Cochrane (1999). Menzly et al. 2004), Bansal and Yaron 2004), and Piazzesi et al. 2007).

\section{Conclusion}

In this paper, we propose an information-theoretic approach as a diagnostic tool for dynamic asset pricing models. The models we consider are characterized by having a pricing kernel that can be factorized into an observable component, consisting of a parametric function of observable variables, and a potentially unobservable one that is model specific. Based on this decomposition of the pricing kernel, we provide three major contributions.

First, using a relative entropy minimization approach, we show how to extract the time series of both the SDF and its unobservable component nonparametrically. Moreover, given a fully observable pricing kernel, this procedure delivers the minimal (in the entropy sense) modification of the SDF that would enable it to price asset returns correctly. Applying this method to the data, we find that the estimated SDF has a clear business-cycle pattern, but also shows significant and sharp reactions to financial market crashes that do not result in economy wide contractions. Moreover, we find that the nonparametrically extracted SDF, independently of the set of assets used for its construction, is substantially (yet not perfectly) correlated with the risk factors proposed in Fama and French 1993). This provides a rationalization of the empirical success of the Fama-French factors in pricing asset returns and suggests that our filtering procedure does successfully identify the unobserved component of the SDF. 
Second, we construct a new set of entropy bounds that build on and improve the ones suggested in the previous literature in that (1) they naturally impose the nonnegativity of the pricing kernel, (2) they are generally tighter and have higher information content, and (3) allow us to utilize the information contained in consumption data and a large cross-section of asset returns jointly.

Third, applying the methodology developed in this paper to a large class of dynamic asset pricing models, we find that the SDFs implied by all of these models poorly correlate with our filtered SDF, require implausibly high levels of risk-aversion to satisfy our entropy bounds, and are all missing a similar component that exhibits significant reactions to financial market crashes that do not result in economy-wide macroeconomic contractions. These results are robust to the choice of test assets used, as well as the frequency of the data.

The methodology developed in this paper is considerably general, and may be applied to any model that delivers well-defined Euler equations like models with heterogenous agents, limited stock market participation, and fragile beliefs.

\section{A. Appendix}

\section{A.1 Maximum Likelihood Analogy}

To formally show the analogy between our estimation approach for the measures $\Psi$ and $Q$ and an MLE procedure, we have to consider the two definition of relative entropy (and corresponding estimators) separately.

First, consider the entropy minimization problem of the type $D(P \| x)$, with $x$ being either the $Q$ or the $\Psi$ measures, used to contruct the estimators in Equations 7 and 6. Let the vector $\mathbf{z}_{t}$ be a sufficient statistic for the state of the economy at time $t$. That is, $\mathbf{z}_{t}$ can be thought of as an augmented state vector (e.g., containing the beginning of period state variables, as well as the time $t$ realizations of the shocks and expectations about the future). Given $\mathbf{z}_{t}$, the equilibrium quantities, such as returns $\mathbf{R}^{e}$ and the sdf $M$, are just a mapping from $\mathbf{z}$ on to the real line, that is,

$$
M(\mathbf{z}): \mathbf{z} \rightarrow \mathbb{R}_{+}, \quad \mathbf{R}^{e}(\mathbf{z}): \mathbf{z} \rightarrow \mathbb{R}^{N}, \quad M_{t} \equiv M\left(\mathbf{z}_{t}\right), \quad \mathbf{R}_{t}^{e} \equiv \mathbf{R}^{e}\left(\mathbf{z}_{t}\right),
$$

where $\mathbf{z}_{t}$ is the time $t$ realization of $\mathbf{z}$.

Equipped with the above definition, we can rewrite the Euler equation 3 as

$$
0=\mathbb{E}\left[\mathbf{R}_{t}^{e} M_{t}\right] \equiv \int \mathbf{R}_{t}^{e} M_{t} d P=\int \mathbf{R}^{e}(\mathbf{z}) M(\mathbf{z}) p(\mathbf{z}) d \mathbf{z},
$$

where $p(\mathbf{z})$ is the pdf associated with the physical measure $P$. Moving to the risk-neutral measure we have

$$
0=\mathbb{E}\left[\mathbf{R}_{t}^{e} M_{t}\right]=\mathbb{E}^{Q}\left[\mathbf{R}_{t}^{e}\right]=\int \mathbf{R}^{e}(\mathbf{z}) q(\mathbf{z}) d \mathbf{z},
$$

where $q(\mathbf{z})$ is the pdf associated with the risk-neutral measure $Q$ and $M=d Q / d P$. Note that

$$
D(P \| Q)=\int \ln \frac{d P}{d Q} d P=\int p(\mathbf{z}) \ln p(\mathbf{z}) d \mathbf{z}-\int p(\mathbf{z}) \ln q(\mathbf{z}) d \mathbf{z} .
$$

Since the first term on the right-hand side of the above expression does not involve $q, D(P \| Q)$ is minimized, with respect to $q$, by choosing the distribution that maximizes the second term, that is,

$$
Q^{*} \equiv \underset{Q}{\arg \min } D(P \| Q) \equiv \underset{q}{\arg \max } \mathbb{E}[\ln q(\mathbf{z})] \text { s.t. } \mathbb{E}^{Q}\left[\mathbf{R}_{t}^{e}\right]=0 .
$$

That is, the minimum entropy estimator in Equation 7 maximizes the expected - risk-neutral - log likelihood. Following Owen 1988, 1991, 2001), approximating the continuous distribution $q(\mathbf{z})$ 
with a multinomial distribution $\left\{q_{t}\right\}_{t=1}^{T}$ that assigns probability weight $q_{t}$ to the time $t$ realizations of $\mathbf{z}$, a NPMLE of $Q$ can be obtained as

$$
\begin{aligned}
& \left\{q_{t}^{*}\right\}_{t=1}^{T}=\operatorname{argmax} \frac{1}{T} \sum_{t=1}^{T} \ln q_{t} \\
& \text { s.t. } q_{t} \in \Delta^{T} \equiv\left\{\left(q_{1}, q_{2}, \ldots, q_{T}\right): q_{t} \geqslant 0, \sum_{t=1}^{T} q_{t}=1\right\} \text { and holds, }
\end{aligned}
$$

provided that

$$
\frac{1}{T} \sum_{t=1}^{T} \ln q_{t} \underset{T \rightarrow \infty}{\stackrel{p .}{\longrightarrow}} \mathbb{E}[\ln q(\mathbf{z})] .
$$

Note also that the NPMLE of $p(\mathbf{z})$ is simply $p_{t}=1 / T \forall t$ (see, e.g., Owen, 1988, 1991, 2001)) which is the maximum entropy distribution. Therefore $q^{*}$ contains all the necessary information to recover the state-price density from the Radon-Nykodin deivative $d Q / d P$.

Similarly, we have that

$$
\begin{aligned}
\Psi^{*} & \equiv \underset{\Psi}{\operatorname{argmin}} D(P \| \Psi) \equiv \underset{\psi}{\operatorname{argmin}} \int p(\mathbf{z}) \ln p(\mathbf{z}) d \mathbf{z}-\int p(\mathbf{z}) \ln \psi(\mathbf{z}) d \mathbf{z} \\
& \equiv \underset{\psi}{\operatorname{argmax}} \mathbb{E}[\ln \psi(\mathbf{z})] \text { s.t. } \mathbb{E}^{\Psi}\left[\mathbf{R}_{t}^{e} m_{t}\right]=0,
\end{aligned}
$$

where $\psi(\mathbf{z})$ is the pdf associated with the measure $\Psi$. That is, the $\Psi^{*}$ estimator in Equation 6 is also an MLE. Moreover, in a very similar fashion, one can show that $\psi^{*} m$ provides a MLE of $q$ under the restrition that the pricing kernel has the multiplicative reppresentation $M=m \psi$.

Hence, the estimates $Q^{*}$ and $\Psi^{*}$ maximize the log likelihoods of the data, but not the physical ones: the risk-neutral log likelihood in the first case and an intermediate one in the second case (and $\Psi^{*}$ also can be interpreted as maximizing the risk-neutral log likelihood under the constraint that $\left.M_{t}=m_{t} \psi_{t}\right)$.

Remark 1. The above implies that, for any equilibrium quantity $A_{t}$, we have that $A_{t} \equiv A\left(\mathbf{z}_{t}\right)$. Hence, the risk-neutral expectation of any function $f($.$) of A$, defined as

$$
\mathbb{E}^{Q}\left[f\left(A_{t}\right)\right] \equiv \int f(A(\mathbf{z})) q(\mathbf{z}) d \mathbf{z},
$$

can be estimated as (see, e.g., Kitamura 2006)

$$
\widehat{\mathbb{E}} \widehat{\left.\left.Q_{\left[f \left(A_{t}\right.\right.}\right)\right]}=\sum_{t=1}^{T} f\left(A_{t}\right) q_{t}^{*},
$$

where $q_{t}^{*}$ is the relative entropy-minimizing risk-neutral measure. For instance, the risk-neutral probability of a recession in a given year that is $\mathbb{E} Q\left[\mathbf{1}_{\text {\{recession in year } t\}}\right]$, where $\mathbf{1}_{\text {\{recession in year } t \text { \} }}$ is an indicator function that takes the value one if time $t$ was an NBER-designated recession and zero otherwise, can be estimated as $\sum_{t=1}^{T} \mathbf{1}_{\text {\{recession in year } t\}} q_{t}^{*}$.

Second, consider the entropy minimization problem of the type $D(x \| P)$ with $x$ being either the $Q$ or the $\Psi$ measures. This alternative definition of relative entropy in Equations 5 and 45 also deliver nonparametric maximum likelihood estimates of the $Q$ and $\Psi$ measures, respectively. We establish this result for $\Psi^{*}$ since for $Q^{*}$ the same result can be shown by a simplified version of the same argument.

To see why the estimation problem in Equation 4 delivers an MLE of $\psi_{t}$, consider the following procedure for constructing (up to a scale) the series $\left\{\psi_{t}\right\}_{t=1}^{T}$. First, given an integer $N>>0$, 
distribute to the various points in time $t=1, \ldots, T$, at random and with equal probabilities, the value $1 / N$ in $N$ independent draws. That is, draw a series of values (probability weights) $\{\tilde{\psi}\}_{t=1}^{T}$ given by

$$
\tilde{\psi}_{t} \equiv \frac{n_{t}}{N},
$$

where $n_{t}$ measures the number of times that the value $1 / N$ has been assigned to time $t$. Second, check whether the drawn series $\{\tilde{\psi}\}_{t=1}^{T}$ satisfies the pricing restriction $\sum_{t=1}^{T} m(\theta, t) R_{t}^{e} \tilde{\psi}_{t}=0$. If it does, use this series as the estimator of $\left\{\psi_{t}\right\}_{t=1}^{T}$, and if it does not draw another series. Obviously, a more efficient way of finding an estimate for $\psi_{t}$ would be to choose the most likely outcome of the above procedure. Noticing that the distribution of the $\tilde{\psi}_{t}$ is, by construction, a multinomial distribution with support given by the data sample, we have that the likelihood of any particular sequence $\left\{\tilde{\psi}_{t}\right\}_{t=1}^{T}$ is

$$
L\left(\left\{\tilde{\psi}_{t}\right\}_{t=1}^{T}\right)=\frac{N !}{n_{1} ! n_{2} ! \ldots n_{T} !} \times T^{-N}=\frac{N !}{N \tilde{\psi}_{1} ! N \tilde{\psi}_{2} ! \ldots N \tilde{\psi}_{T} !} \times T^{-N} .
$$

Therefore, the most likely value of $\left\{\tilde{\psi}_{t}\right\}_{t=1}^{T}$ maximizes the log likelihood

$$
\ln L\left(\left\{\tilde{\psi}_{t}\right\}_{t=1}^{T}\right) \propto \frac{1}{N}\left(\ln N !-\sum_{t=1}^{T} \ln \left(N \tilde{\psi}_{t} !\right)\right) .
$$

Since the above procedure of assigning probability weights will become more and more accurate as $N$ grows bigger, we would ideally like to have $N \rightarrow \infty$. But in this case one can show that22

$$
\lim _{N \rightarrow \infty} \ln L\left(\left\{\tilde{\psi}_{t}\right\}_{t=1}^{T}\right)=-\sum_{t=1}^{T} \tilde{\psi}_{t} \ln \tilde{\psi}_{t} .
$$

Therefore, taking into account the constraint for the pricing kernel, the MLE of $\psi_{t}$ solves

$$
\left\{\hat{\psi}_{t}\right\}_{t=1}^{T} \equiv \operatorname{argmax}-\sum_{t=1}^{T} \tilde{\psi}_{t} \ln \tilde{\psi}_{t}, \quad \text { s.t. }\left\{\widetilde{\psi}_{t}\right\}_{t=1}^{T} \in \Delta^{T}, \sum_{t=1}^{T} m(\theta, t) \mathbf{R}_{t}^{e} \widetilde{\psi}_{t}=\mathbf{0} .
$$

But the solution of the above MLE problem is also the solution of the relative entropy minimization problem in Equation 4 (see, e.g., Csiszar 1975). That is, the KLIC minimization is equivalent to maximizing the likelihood in an unbiased procedure for finding the $\psi_{t}$ component of the pricing kernel.

\section{A.2 Additional Bounds and Derivations}

Remark 2 ( $H J$-bounds as approximated $Q$-bounds). Let $p$ and $q$ denote the densities of the state $x$ associated, respectively, with the physical, $P$, and the risk-neutral, $Q$, probability measures 23 Assuming that

22 Recall that from Stirling's formula, we have

$$
\lim _{N \psi_{t} \rightarrow \infty} \frac{N \tilde{\psi}_{t} !}{\sqrt{2 \pi N \tilde{\psi}_{t}}\left(\frac{N \psi_{t}}{e}\right)^{N \tilde{\psi}_{t}}}=1 .
$$

23 For expositional simplicity, we focus on a scalar state variable, but the result is straightforward to extend to a vector state. 
A.1 $q$ and $p$ are twice continuously differentiable and that there exists a $\mu_{p}<\infty$ and a $\mu_{q}<\infty$ such that:

A.2 (Existence of maxima)

$$
\left.\frac{\partial \ln p}{\partial x}\right|_{x=\mu_{p}}=0,\left.\frac{\partial \ln q}{\partial x}\right|_{x=\mu_{q}}=0
$$

A.3 (Finite second moments)

$$
-\left[\left.\frac{\partial^{2} \ln p}{\partial x^{2}}\right|_{x=\mu_{p}}\right]^{-1} \equiv \sigma_{p}^{2}<\infty,-\left[\left.\frac{\partial^{2} \ln q}{\partial x^{2}}\right|_{x=\mu_{q}}\right]^{-1} \equiv \sigma_{q}^{2}<\infty .
$$

We have that, in the limit of the small time interval, a second-order approximation of the Q-bounds yield 24

$$
\begin{aligned}
& D\left(P \| \frac{M_{t}}{\bar{M}}\right) \propto \operatorname{Var}\left(M_{t}\right), \\
& D\left(\frac{M_{t}}{\bar{M}} \| P\right) \propto \operatorname{Var}\left(M_{t}\right) .
\end{aligned}
$$

Proof. [Proof of Remark 2 The densities associated, respectively, with the physical probability measure $P$ and the risk-neutral measure $Q$ are denoted $p$ and $q$. We can then rewrite the $Q 1$ and $Q 2$ bounds, respectively, as

$$
D\left(P \| \frac{M_{t}}{\bar{M}}\right) \equiv \int \ln \frac{d P}{d Q} d P=\int p \ln \frac{p}{q} d x
$$

and

$$
D\left(\frac{M_{t}}{\bar{M}} \| P\right) \equiv \int \frac{d Q}{d P} \ln \frac{d Q}{d P} d P=\int \ln \frac{d Q}{d P} d Q=\int q \ln \frac{q}{p} d x .
$$

Given conditions A.1-A.3, we have from a second-order Taylor approximation that

$$
\begin{aligned}
& \left.\ln q \propto \frac{1}{2} \frac{\partial^{2} \ln q}{\partial x^{2}}\right|_{x=\mu_{q}}\left(x-\mu_{q}\right)^{2} \equiv-\frac{1}{2} \frac{\left(x-\mu_{q}\right)^{2}}{\sigma_{q}^{2}} . \\
& \left.\ln p \propto \frac{1}{2} \frac{\partial^{2} \ln p}{\partial x^{2}}\right|_{x=\mu_{p}}\left(x-\mu_{p}\right)^{2} \equiv-\frac{1}{2} \frac{\left(x-\mu_{p}\right)^{2}}{\sigma_{p}^{2}} .
\end{aligned}
$$

That is, $q$ and $p$ are approximately (to a second-order) Gaussian

$$
q \approx N\left(\mu_{q} ; \sigma_{q}^{2}\right), \quad p \approx N\left(\mu_{p} ; \sigma_{p}^{2}\right)
$$

Note also that in the limit of the small time interval, by the diffusion invariance principle, we have $\sigma_{q}^{2}=\sigma_{p}^{2}=\sigma^{2}$. Therefore, plugging the above approximation into Equation A8, we have that in the

24 For the $Q 2$ bound only, using the dual objective function of the entropy minimization problem, Stutze 1995, provides an approximation result similar to the one in Equation A7 that is valid when the variance bound is sufficiently small. Moreover, for the case of Gaussian iid returns, Kitamura and Stutzen 2002) show that the approximation of the $Q 2$ bound in Equation $\mathrm{A} 7$ is exact. 
limit of the small time interval

$$
\begin{aligned}
\int p \ln \frac{p}{q} d x & \approx \int\left[-\frac{1}{2} \frac{\left(x-\mu_{p}\right)^{2}}{\sigma^{2}}+\frac{1}{2} \frac{\left(x-\mu_{q}\right)^{2}}{\sigma^{2}}\right] p d x \\
& =\frac{1}{2 \sigma^{2}}\left[-\sigma^{2}+\int\left(x-\mu_{q}\right)^{2} p d x\right] \\
& =\frac{1}{2 \sigma^{2}}\left\{-\sigma^{2}+\int\left[\left(x-\mu_{p}\right)^{2}+\left(\mu_{p}-\mu_{q}\right)^{2}\right.\right. \\
& \left.\left.+2\left(\mu_{p}-\mu_{q}\right)\left(x-\mu_{p}\right)\right] p d x\right\} \\
& =\frac{1}{2 \sigma^{2}}\left(\mu_{p}-\mu_{q}\right)^{2}=\frac{1}{2 \sigma^{2}} \sigma^{2} \sigma_{\xi}^{2}=\frac{1}{2} \sigma_{\xi}^{2}
\end{aligned}
$$

where the density $\xi$ is a (strictly positive) martingale defined by $\xi \equiv \frac{d Q}{d P}$, and the one to the last equality comes from the change of drift implied by the Girsanov's Theorem (see, e.g., Duffie, 2005, Appendix D)).

Similarly, from Equation $\mathrm{A} 9$ we have

$$
\int q \ln \frac{q}{p} d x=\frac{1}{2} \sigma_{\xi}^{2}
$$

Since $Q$ and $P$ are equivalent measures, $M_{t} \propto \xi_{t}$. Therefore, in the limit of the small time interval $\operatorname{Var}\left(M_{t}\right) \propto \sigma_{\xi}^{2}$, implying

$$
D\left(P \| \frac{M_{t}}{\bar{M}}\right) \propto \operatorname{Var}\left(M_{t}\right), \quad D\left(\frac{M_{t}}{\bar{M}} \| P\right) \propto \operatorname{Var}\left(M_{t}\right) .
$$

Definition 5 ( Volatility bound for $\psi_{t}$ ). For each $E\left[\psi_{t}\right]=\bar{\psi}$, the minimum variance $\psi_{t}$ is

$$
\psi_{t}^{*}(\bar{\psi}) \underset{\left\{\psi_{t}(\bar{\psi})\right\}_{t=1}^{T}}{\operatorname{argmin}} \sqrt{\operatorname{Var}\left(\psi_{t}(\bar{\psi})\right)} \text { s.t. } \mathbf{0}=\mathbb{E}\left[\mathbf{R}_{t}^{e} m(\theta, t) \psi_{t}(\bar{\psi})\right],
$$

and any candidate SDF must satisfy the condition $\operatorname{Var}\left(\psi_{t}\right) \geq \operatorname{Var}\left(\psi_{t}^{*}(\bar{\psi})\right)$.

The solution of the above minimization for a given $\theta$ is

$$
\psi_{t}^{*}(\bar{\psi})=\bar{\psi}+\left(\mathbf{R}_{t}^{e} m(\theta, t)-\mathbb{E}\left[\mathbf{R}_{t}^{e} m(\theta, t)\right]\right)^{\prime} \beta_{\bar{\psi}},
$$

where $\beta_{\bar{\psi}}=\operatorname{Var}\left(\mathbf{R}_{t}^{e} m(\theta, t)\right)^{-1}\left(-\bar{\psi} \mathbb{E}\left[\mathbf{R}_{t}^{e} m(\theta, t)\right]\right)$ and the lower volatility bound is given by

$$
\sigma_{\psi^{*}} \equiv \sqrt{\operatorname{Var}\left(\psi_{t}^{*}(\bar{\psi})\right)}=\bar{\psi} \sqrt{\mathbb{E}\left[\mathbf{R}_{t}^{e} m(\theta, t)\right]^{\prime} \operatorname{Var}\left(\mathbf{R}_{t}^{e} m(\theta, t)\right)^{-1} \mathbb{E}\left[\mathbf{R}_{t}^{e} m(\theta, t)\right]} .
$$

\section{A.3 HJ Kernel Versus Minimum Entropy Kernel}

We compute the minimum adjustment to the CCAPM SDF required to make it an admissible pricing kernel using (1) the HJD approach and (2) the relative entropy minimization approach. Table A1 presents the moments of the resultant SDFs extracted using both these approaches for a variety of different sets of test assets. 
Table A1

Moments of SDF, 1947:Q1-2009:Q4

\begin{tabular}{lccc} 
& $\sigma\left(M_{t}^{*}\right)$ & $S k\left(M_{t}^{*}\right)$ & $\operatorname{Kurt}\left(M_{t}^{*}\right)$ \\
\hline A. HJD kernel & & & \\
\hline 25 FF & 0.45 & -0.01 & 3.12 \\
Market & 0.22 & 0.61 & 3.91 \\
10 momentum & 0.41 & 0.05 & 3.41 \\
10 industry & 0.32 & 0.54 & 4.21 \\
\hline B. Minimum entropy kernel & & & $28.4 / 9.31$ \\
\hline 25 FF & $0.91 / 0.71$ & $4.53 / 2.07$ & $19.1 / 8.59$ \\
Market & $0.26 / 0.24$ & $3.14 / 1.84$ & $22.0 / 7.22$ \\
10 momentum & $0.69 / 0.57$ & $3.82 / 1.78$ & $39.6 / 11.8$ \\
10 industry & $0.45 / 0.39$ & $5.08 / 2.32$ &
\end{tabular}

The table reports the moments of the SDF computed using the (1) the HJD minimum linear adjustment (panel A) and (2) the minimum relative entropy log-linear adjustment (panel B). The test assets used in the estimation of the minimum adjustment consist of the 25 size and book-to-market-equity-sorted portfolios (Row 1), the market portfolio (Row 2), the ten momentum-sorted portfolios (Row 3), and the ten industry-sorted portfolios (Row 4). The data are quarterly for 1947:Q1-2009:Q4.

\section{A.4 Data Description}

At the quarterly frequency, we use six different sets of assets: (1) the market portfolio, (2) the 25 Fama-French portfolios, (3) the 10 size-sorted portfolios, (4) the 10 book-to-market-equity-sorted portfolios, (5) the ten momentum-sorted portfolios, and (6) the ten industry-sorted portfolios. At the annual frequency, we use the same sets of assets, except the 25 Fama-French portfolios are replaced by the 6 portfolios formed by sorting stocks on the basis of size and book-to-market-equity because of the small time-series dimension available at the annual frequency.

Our proxy for the market return is the Center for Research in Security Prices (CRSP) valueweighted index of all stocks on the NYSE, AMEX, and NASDAQ. The proxy for the risk-free rate is the one-month Treasury-bill rate obtained from the CRSP files. The returns on all the portfolios are obtained from Kenneth French's data library. Quarterly (annual) returns for the above assets are computed by compounding monthly returns within each quarter (year), and converted to real using the personal consumption deflator. Excess returns on the assets are then computed by subtracting the risk-free rate.

Finally, for each dynamic asset pricing model, the information bounds and the nonparametrically extracted and model-implied time series of the SDF depend on consumption data. For the standard Consumption-CAPM of Breeden 1979) and Rubinstein 1976), the external habit models of Campbell and Cochrane 1999) and Menzly et al. 2004), and the long-run risks model of Bansal and Yaron 2004), we use per capita real personal consumption expenditures on nondurable goods from the National Income and Product Accounts (NIPA). We make the standard "end-of-period" timing assumption that consumption during quarter $t$ takes place at the end of the quarter. For the housing model of Piazzesi et al. 2007), aggregate consumption is measured as expenditures on nondurables and services excluding housing services.

A.5 Extracting the Model-Implied SDF for the Menzly et al. (2004) Model The SDF in this model is given by

$$
M_{t}=\delta\left(C_{t} / C_{t-1}\right)^{-\gamma}\left(S_{t} / S_{t-1}\right)^{-\gamma},
$$

where $\delta$ is the subjective time discount factor, $\gamma$ is the utility curvature parameter, $S_{t}=\frac{C_{t}-X_{t}}{C_{t}}$ denotes the surplus consumption ratio, and $X_{t}$ is the habit component.

The inverse surplus, $Y_{t}=\frac{1}{S_{t}}$, follows a mean-reverting process:

$$
d Y_{t}=k\left(\bar{Y}-Y_{t}\right) d t-\alpha\left(Y_{t}-\lambda\right) \sigma_{c} d B_{t} .
$$


Therefore, using Ito's lemma, $s_{t} \equiv \ln \left(S_{t}\right)=-\ln \left(Y_{t}\right)$ follows the process

$$
\begin{aligned}
d s_{t} & =-\frac{1}{Y_{t}} d Y_{t}+\frac{1}{2 Y_{t}^{2}}\left(d Y_{t}\right)^{2} \\
& =-\frac{1}{Y_{t}} k\left(\bar{Y}-Y_{t}\right) d t+\frac{1}{Y_{t}} \alpha\left(Y_{t}-\lambda\right) \sigma_{c} d B_{t}+\frac{1}{2 Y_{t}^{2}} \alpha^{2}\left(Y_{t}-\lambda\right)^{2} \sigma_{c}^{2} d t \\
& =\left[k\left(1-\bar{Y} S_{t}\right)+\frac{1}{2} \alpha^{2}\left(1-\lambda S_{t}\right)^{2} \sigma_{c}^{2}\right] d t+\alpha\left(1-\lambda S_{t}\right) \sigma_{c} d B_{t} .
\end{aligned}
$$

Therefore, discretizing the process, we have

$$
\Delta s_{t+1}=k\left(1-\bar{Y} S_{t}\right)+\frac{1}{2} \alpha^{2}\left(1-\lambda S_{t}\right)^{2} \sigma_{c}^{2}+\alpha\left(1-\lambda S_{t}\right) \sigma_{c} \varepsilon_{t+1},
$$

where $\varepsilon_{t+1} \sim$ i.i.d. $N(0,1)$.

Now, the Euler equation for the return on the aggregate consumption claim is

$$
E_{t}\left(e^{m_{t+1}+r_{c, t t+1}}\right)=1,
$$

where $r_{c, t+1}$ denotes the continuously compounded return on the consumption claim. We rely on log-linear approximations for $r_{c, t+1}$, as in Campbell and Shiller (1988):

$$
r_{c, t+1}=\kappa_{0}+\kappa_{1} z_{t+1}-z_{t}+\Delta c_{t+1},
$$

where $z_{t}$ is the $\log$ price-consumption ratio. In Equation $\mathrm{A12}, \kappa_{1}=\frac{e^{\bar{z}}}{1+e^{\bar{z}}}$ and $\kappa_{0}=\log \left(1+e^{e^{\bar{z}}}\right)-$ $\kappa_{1} e^{\bar{z}}$, where $\bar{z}$ denotes the long-run mean of the log price-consumption ratio. We conjecture that $z_{t}$ is affine in the single state variable $s_{t}$ :

$$
z_{t}=A_{0}+A_{1} s_{t} .
$$

To verify the conjecture and also solve for $A_{0}$ and $A_{1}$, we substitute the expressions for $r_{c, t+1}$ and $z_{t}$ from Equations $\mathrm{A12}$ and $\mathrm{A13}$, respectively, into the Euler equation $\mathrm{A11}$ :

$$
\Rightarrow E_{t}\left(\operatorname { e x p } \left\{\begin{array}{c}
E_{t}\left(\exp \left\{\ln \delta-\gamma \Delta c_{t+1}-\gamma \Delta s_{t+1}+\kappa_{0}+\kappa_{1} z_{c, t+1}-z_{t}+\Delta c_{t+1}\right\}\right)=1, \\
\left.+\kappa_{0}+\kappa_{1} A_{0}+\kappa_{1} A_{1}\left[\begin{array}{c}
\left.\ln \left(1-\bar{Y} S_{t}\right)+\frac{1}{2} \alpha^{2}\left(1-\lambda S_{t}\right)^{2} \sigma_{c}^{2}+\alpha\left(1-\lambda S_{t}\right) \sigma_{c} \varepsilon_{t+1}+s_{t}\right] \\
-A_{0}-A_{1} s_{t}+\mu_{c}+\sigma_{c} \varepsilon_{t+1}
\end{array}\right\}\right)=1 .
\end{array}\right.\right.
$$

Using the properties of conditionally lognormal random variables, we have

$$
\begin{aligned}
0= & \ln \delta-\gamma \mu_{c}-\gamma k+\gamma k \bar{Y} S_{t}-\frac{1}{2} \gamma \alpha^{2} \sigma_{c}^{2}-\frac{1}{2} \gamma \alpha^{2} \lambda^{2} \sigma_{c}^{2} S_{t}^{2}+\gamma \alpha^{2} \sigma_{c}^{2} \lambda S_{t}+\kappa_{0}+\kappa_{1} A_{0} \\
& +\kappa_{1} A_{1} k-\kappa_{1} A_{1} k \bar{Y} S_{t}+\frac{1}{2} \kappa_{1} A_{1} \alpha^{2} \sigma_{c}^{2}+\frac{1}{2} \kappa_{1} A_{1} \alpha^{2} \sigma_{c}^{2} \lambda^{2} S_{t}^{2}-\kappa_{1} A_{1} \alpha^{2} \sigma_{c}^{2} \lambda S_{t}+\kappa_{1} A_{1} S_{t} \\
& -A_{0}-A_{1} s_{t}+\mu_{c}+\frac{1}{2}\left[-\gamma-\gamma \alpha\left(1-\lambda S_{t}\right)+\kappa_{1} A_{1} \alpha\left(1-\lambda S_{t}\right)+1\right]^{2} \sigma_{c}^{2},
\end{aligned}
$$

which implies

$$
\begin{aligned}
0= & \left(\begin{array}{c}
\ln \delta-\gamma \mu_{c}-\gamma k-\frac{1}{2} \gamma \alpha^{2} \sigma_{c}^{2}+\kappa_{0}+\kappa_{1} A_{0}+\kappa_{1} A_{1} k+\frac{1}{2} \kappa_{1} A_{1} \alpha^{2} \sigma_{c}^{2} \\
-A_{0}+\mu_{c}+\frac{1}{2}\left[-\gamma-\gamma \alpha+\kappa_{1} A_{1} \alpha+1\right]^{2} \sigma_{c}^{2}
\end{array}\right) \\
& +\left(\begin{array}{c}
\gamma k \bar{Y}+\gamma \alpha^{2} \sigma_{c}^{2} \lambda-\kappa_{1} A_{1} k \bar{Y}-\kappa_{1} A_{1} \alpha^{2} \sigma_{c}^{2} \lambda \\
+\left[\gamma \alpha \lambda-\kappa_{1} A_{1} \alpha \lambda\right]\left[-\gamma-\gamma \alpha+\kappa_{1} A_{1} \alpha+1\right] \sigma_{c}^{2}
\end{array}\right) S_{t} \\
& +\left(\kappa_{1} A_{1}-A_{1}\right) s_{t} \\
& +\left(-\frac{1}{2} \gamma \alpha^{2} \lambda^{2} \sigma_{c}^{2}+\frac{1}{2} \kappa_{1} A_{1} \alpha^{2} \sigma_{c}^{2} \lambda^{2}+\frac{1}{2}\left(\gamma \alpha \lambda-\kappa_{1} A_{1} \alpha \lambda\right)^{2} \sigma_{c}^{2}\right) S_{t}^{2} .
\end{aligned}
$$


Table A2

Bounds for RRA when all parameters are allowed to vary

$H J$-bound $Q 1 / Q 2$-bounds

$M 1 / M 2$-bounds

$\Psi 1 / \Psi 2$-bounds

A. Quarterly data, 1947:Q1-2009:Q4

\begin{tabular}{lrccc}
\hline$C C$ & 2.2 & $4.0 / 3.8$ & $4.0 / 3.8$ & $4.3 / 4.2$ \\
$M S V$ & 29.0 & $36.2 / 35.9$ & $38.0 / 38.1$ & $50.9 / 52.5$ \\
$B Y$ & 3.0 & $4.0 / 4.0$ & $4.0 / 4.0$ & $4.0 / 4.0$ \\
$P S T$ & 19.1 & $25.2 / 24.0$ & $25.4 / 24.1$ & $24.1 / 23.1$
\end{tabular}

B. Annual data, 1929-2009

\begin{tabular}{lrccc}
\hline$C C$ & 0.1 & $0.1 / 0.1$ & $0.1 / 0.1$ & $0.1 / 0.1$ \\
$M S V$ & 11.3 & $18.6 / 16.2$ & $19.3 / 17.2$ & $28.6 / 27.2$ \\
$B Y$ & 4.0 & $4.0 / 4.0$ & $4.0 / 4.0$ & $4.0 / 4.0$ \\
$P S T$ & 4.3 & $6.8 / 5.8$ & $6.8 / 5.8$ & $6.3 / 5.4$ \\
\hline
\end{tabular}

The table reports the minimum values of the utility curvature parameter $\gamma$ at which the model-implied SDF satisfies the HJ (Column 1), Q (Column 2), M (Column 3), and $\Psi$ (Column 4) bounds using quarterly data for 1947:Q1-2009:Q4 (panel A) and annual data for 1929-2009 (panel B). Columns 2-4 has two entries in each cell that correspond to whether the filtered $\psi^{*}$-component of the SDF and, therefore, the filtered SDF are estimated using Equation 6, reported on the left, or Equation 4, reported on the right. $C C, M S V, B Y$, and $P S T$ denote, respectively, the models of Campbell and Cochrane 1999), Menzly et al, 2004, Bansal and Yaron 2004), and Piazzesi et al, 2007).

Using the approximations $s_{t} \approx S_{t}-1$ and $S_{t}^{2} \approx-\bar{S}^{2}+2 \bar{S} S_{t}$, we have

$$
\begin{aligned}
0= & \left(\begin{array}{c}
\ln \delta-\gamma \mu_{c}-\gamma k-\frac{1}{2} \gamma \alpha^{2} \sigma_{c}^{2}+\kappa_{0}+\kappa_{1} A_{0}+\kappa_{1} A_{1} k+\frac{1}{2} \kappa_{1} A_{1} \alpha^{2} \sigma_{c}^{2} \\
-A_{0}+\mu_{c}+\frac{1}{2}\left[-\gamma-\gamma \alpha+\kappa_{1} A_{1} \alpha+1\right]^{2} \sigma_{c}^{2}
\end{array}\right) \\
& +\left(\begin{array}{c}
\gamma k \bar{Y}+\gamma \alpha^{2} \sigma_{c}^{2} \lambda-\kappa_{1} A_{1} k \bar{Y}-\kappa_{1} A_{1} \alpha^{2} \sigma_{c}^{2} \lambda \\
+\left[\gamma \alpha \lambda-\kappa_{1} A_{1} \alpha \lambda\right]\left[-\gamma-\gamma \alpha+\kappa_{1} A_{1} \alpha+1\right] \sigma_{c}^{2}
\end{array}\right) S_{t} \\
& +\left(\kappa_{1} A_{1}-A_{1}\right)\left(S_{t}-1\right) \\
& +\left(-\frac{1}{2} \gamma \alpha^{2} \lambda^{2} \sigma_{c}^{2}+\frac{1}{2} \kappa_{1} A_{1} \alpha^{2} \sigma_{c}^{2} \lambda^{2}+\frac{1}{2}\left(\gamma \alpha \lambda-\kappa_{1} A_{1} \alpha \lambda\right)^{2} \sigma_{c}^{2}\right)\left(-\bar{S}^{2}+2 \bar{S} S_{t}\right) .
\end{aligned}
$$

We use the method of undetermined coefficients and set to zero the constant term and the coefficient of $S_{t}$ to obtain two equations in the two unknowns $A_{0}$ and $A_{1}$ :

$$
\begin{aligned}
0= & \left(\begin{array}{c}
\ln \delta-\gamma \mu_{c}-\gamma k-\frac{1}{2} \gamma \alpha^{2} \sigma_{c}^{2}+\kappa_{0}+\kappa_{1} A_{0}+\kappa_{1} A_{1} k+\frac{1}{2} \kappa_{1} A_{1} \alpha^{2} \sigma_{c}^{2} \\
-A_{0}+\mu_{c}+\frac{1}{2}\left[-\gamma-\gamma \alpha+\kappa_{1} A_{1} \alpha+1\right]^{2} \sigma_{c}^{2}
\end{array}\right) \\
& -\left(\kappa_{1} A_{1}-A_{1}\right) \\
& -\left(-\frac{1}{2} \gamma \alpha^{2} \lambda^{2} \sigma_{c}^{2}+\frac{1}{2} \kappa_{1} A_{1} \alpha^{2} \sigma_{c}^{2} \lambda^{2}+\frac{1}{2}\left(\gamma \alpha \lambda-\kappa_{1} A_{1} \alpha \lambda\right)^{2} \sigma_{c}^{2}\right) \bar{S}^{2} .
\end{aligned}
$$

and

$$
\begin{aligned}
0= & \left(\begin{array}{c}
\gamma k \bar{Y}+\gamma \alpha^{2} \sigma_{c}^{2} \lambda-\kappa_{1} A_{1} k \bar{Y}-\kappa_{1} A_{1} \alpha^{2} \sigma_{c}^{2} \lambda \\
+\left[\gamma \alpha \lambda-\kappa_{1} A_{1} \alpha \lambda\right]\left[-\gamma-\gamma \alpha+\kappa_{1} A_{1} \alpha+1\right] \sigma_{c}^{2}
\end{array}\right) \\
& +\left(\kappa_{1} A_{1}-A_{1}\right) \\
& +2 \bar{S}\left(-\frac{1}{2} \gamma \alpha^{2} \lambda^{2} \sigma_{c}^{2}+\frac{1}{2} \kappa_{1} A_{1} \alpha^{2} \sigma_{c}^{2} \lambda^{2}+\frac{1}{2}\left(\gamma \alpha \lambda-\kappa_{1} A_{1} \alpha \lambda\right)^{2} \sigma_{c}^{2}\right) .
\end{aligned}
$$


Solving the equations for $A_{0}$ and $A_{1}$ gives the equilibrium solution for the log price-consumption ratio in Equation A13. Note that Equation A15 implies a quadratic equation for $A_{1}$ :

$$
\begin{aligned}
0= & \left(-\kappa_{1}^{2} \alpha^{2} \lambda \sigma_{c}^{2}+\bar{S} \kappa_{1}^{2} \alpha^{2} \lambda^{2} \sigma_{c}^{2}\right) A_{1}^{2} \\
& +\left(\begin{array}{c}
\left.-\kappa_{1} k \bar{Y}-\kappa_{1} \alpha^{2} \sigma_{c}^{2} \lambda+\gamma \alpha \lambda \kappa_{1} \alpha \sigma_{c}^{2}-\kappa_{1} \alpha \lambda[-\gamma-\gamma \alpha+1] \sigma_{c}^{2}\right) A_{1} \\
+\kappa_{1}-1+\bar{S} \kappa_{1} \alpha^{2} \sigma_{c}^{2} \lambda^{2}-2 \bar{S} \gamma \alpha^{2} \lambda \sigma_{c}^{2} \kappa_{1}
\end{array}\right. \\
& +\left(\gamma k \bar{Y}+\gamma \alpha^{2} \sigma_{c}^{2} \lambda+\gamma \alpha \lambda[-\gamma-\gamma \alpha+1] \sigma_{c}^{2}-\bar{S} \gamma \alpha^{2} \lambda^{2} \sigma_{c}^{2}+\bar{S} \gamma^{2} \alpha^{2} \lambda^{2} \sigma_{c}^{2}\right) .
\end{aligned}
$$

We choose the smaller root of the quadratic equation as the economically meaningful solution because it implies a positive relation between the log price-consumption ratio and the surplus consumption ratio, unlike the bigger root that implies a negative relation between the variables.

We proxy the log price-consumption ratio using the observable log price-dividend ratio and use Equation th13 to extract the time series of the state variable $s_{t}$. This extracted time series can then be used to obtain the time series of the model-implied SDF and its missing component.

Note that the model is calibrated at the quarterly frequency. Since we evaluate the empirical plausibility of models at the quarterly, as well as annual, frequencies, we obtain the annual estimates of the model parameters as follows. As a first step, we simulate a long sample (five million observations) of the state variable $Y$ from

$$
\Delta Y_{q, \tau+1}=k_{q}\left(\bar{Y}_{q}-Y_{q, \tau}\right)-\alpha_{q}\left(Y_{q, \tau}-\lambda_{q}\right) \sigma_{q, c} \varepsilon_{\tau+1}, \quad \varepsilon_{\tau+1} \sim i . i . d . N(0,1),
$$

treating the calibrated quarterly parameter values as the truth. The subscript $q$ in the above equation denotes quarterly. As a second step, we aggregate the simulated data into annual nonoverlapping observations:

$$
\begin{aligned}
Y_{a, t} & =Y_{q, \tau}+Y_{q, \tau-1}+Y_{q, \tau-2}+Y_{q, \tau-3}, \text { for } \tau=1,2,3, \ldots \\
\Delta Y_{a, t+1} & =Y_{a, t+1}-Y_{a, t},
\end{aligned}
$$

where $\tau$ denotes quarter $\tau$ and $t$ denotes year $t$. As a final step, we estimate the model parameters at the annual frequency from the equation

$$
\Delta Y_{a, t+1}=k_{a}\left(\bar{Y}_{a}-Y_{a, t}\right)-\alpha_{a}\left(Y_{a, t}-\lambda_{a}\right) \sigma_{a, c} e_{t+1}, \quad \varepsilon_{t+1} \sim \text { i.i.d.N }(0,1),
$$

treating the state variable $Y_{a, t}$ as observed and using the method of moments approach. This step produces the following annual estimates of the parameters: $\bar{Y}_{a}=33.99531, k_{a}=.8689003$, $\alpha_{a}=3.49499, \lambda_{a}=29.843719$. The mean , $\mu_{a, c}$, and volatility , $\sigma_{a, c}$, of aggregate consumption growth are set equal to their sample values.

\section{A.6 Extracting the Model-Implied SDF for the Menzly et al. (2004) Model}

The SDF in this model is given by

$$
M_{t+1}=\delta^{\theta}\left(\frac{C_{t+1}}{C_{t}}\right)^{-\frac{\theta}{\rho}} R_{c, t+1}^{\theta-1},
$$

where $R_{c, t+1}$ is the unobservable gross return on an asset that delivers aggregate consumption as its dividend each period.

Using the Campbell-Shiller log-linearization for $r_{c, t+1} \equiv \ln \left(R_{c, t+1}\right)$ :

$$
r_{c, t+1}=\kappa_{0}+\kappa_{1} z_{t+1}-z_{t}+\Delta c_{t+1},
$$

where $z_{t}$ is the log price-consumption ratio. Noting that the model implies that the equilibrium $z_{t}=A_{0}+A_{1} x_{t}+A_{2} \sigma_{t}^{2}$, we have

$$
\begin{aligned}
\ln M_{t} & =\left[\theta \ln \delta+(\theta-1)\left(\kappa_{0}+\kappa_{1} A_{0}-A_{0}\right)\right]-\gamma \Delta c_{t+1} \\
& +(\theta-1) \kappa_{1} A_{1} x_{t+1}+(\theta-1) \kappa_{1} A_{2} \sigma_{t+1}^{2}-(\theta-1) A_{1} x_{t}-(\theta-1) A_{2} \sigma_{t}^{2} .
\end{aligned}
$$

This is Equation 24 in the text. To obtain the time series of the SDF and its $\psi$ component, we extract the state variables, $x_{t}$ and $\sigma_{t}^{2}$, from observed data using two different procedures. 
First, we extract the state variables from consumption data. To do so, we assume the same time-series specification for the aggregate consumption growth process as in Bansal and Yaron 2004), with a square-root process for the variance (as in Hansen et al. 2007) as the only exception:

$$
\begin{aligned}
\Delta c_{t+1} & =\mu+x_{t}+\sigma_{t} \eta_{t+1}, \\
x_{t+1} & =\rho x_{t}+\phi_{e} \sigma_{t} e_{t+1}, \\
\sigma_{t+1}^{2} & =\sigma^{2}\left(1-v_{1}\right)+v_{1} \sigma_{t}^{2}+\sigma_{w} \sigma_{t} w_{t+1} .
\end{aligned}
$$

Note that the Bansal and Yaron 2004 model is calibrated at the monthly frequency with the monthly parameter values being $\mu=.0015, \rho=.979, \phi_{e}=.044, \sigma=.0078, v_{1}=.987$, and $\sigma_{w}=.00029487$. We need to extract the quarterly state variables $x_{t, q}$ and $\sigma_{t, q}^{2}$. As a first step, we simulate a long sample (five million observations) from the above system, treating the given parameter values as the truth and retaining the simulated state variables. As a second step, we aggregate the simulated data into quarterly nonoverlapping observations:

$$
\begin{aligned}
\Delta c_{t, q} & =\Delta c_{t}+\Delta c_{t-1}+\Delta c_{t-2}, \text { for } t=3,6,9, \ldots \\
x_{t, q} & =x_{t}+x_{t-1}+x_{t-2} \\
\sigma_{t, q}^{2} & =\sigma_{t}^{2}+\sigma_{t-1}^{2}+\sigma_{t-2}^{2} .
\end{aligned}
$$

As a third step, we estimate the model parameters in Equations A17-A19 using these quarterly observations and treating the state variables as observed. This step produces the following quarterly estimates of the parameters:

$$
\begin{aligned}
\rho_{q} & =\rho_{m}^{3}=.9383137, \\
v_{1, q} & =v_{1, m}^{3}=.9615048, \\
\mu_{q} & =3 \times \mu_{m}=.0045, \\
\sigma_{q}^{2} & =\operatorname{Mean}\left(\sigma_{t, q}^{2}\right)=.0001822490, \\
\phi_{e, q} & =\sqrt{\frac{\operatorname{Var}\left(x_{t+1, q}-\rho_{q} x_{t, q}\right)}{\sigma_{q}^{2}}}=.1084845, \\
\sigma_{w, q} & =\sqrt{\frac{\operatorname{Var}\left(\sigma_{t+1, q}^{2}-\sigma_{q}^{2}\left(1-v_{1, q}\right)-v_{1, q} \sigma_{t, q}^{2}\right)}{\sigma_{q}^{2}}}=0.0007328592,
\end{aligned}
$$

where the variables with subscript $m$ are the monthly calibrated values, and the means and variances are the ones obtained in the simulated sample. As a fourth step, we run a Bayesian smoother through the historical quarterly consumption growth treating the quarterly parameters as being known with certainty. The smoother produces estimates of the quarterly state variables $\hat{x}_{t, q}$ and $\hat{\sigma}_{t, q}^{2}$.

The same steps can be applied to obtain the parameter estimates and, therefore, the time series of the state variables at the annual frequency. In this case, we have $\rho_{a}=.7751617, v_{1, a}=.8546845$, $\mu_{a}=.018, \sigma_{a}^{2}=.0007299038, \phi_{e, a}=.3853643$, and $\sigma_{w, a}=.00270020$.

Using the point estimates of the parameters and the extracted time series of the state variables at the relevant frequency, the SDF and its missing $\psi$ component are obtained from Equation 24].

Our second procedure for extracting the state variables relies on asset market data. For the $\log$-linearized version of the model, the observable log market-wide price-dividend ratio, $z_{m, t}$, and the $\log$ gross risk-free rate, $r_{f . t}$, are affine functions of the state variables, $x_{t}$ and $\sigma_{t}^{2}$. Therefore, Constantinides and Ghosh 2011) argue that these affine functions may be inverted to express 
Table A3

Bounds for RRA, quarterly data, 1947:Q1-2009:Q4

$H J$-bound

$Q 1 / Q 2$-bounds

$M 1 / M 2$-bounds

$\Psi 1 / \Psi 2$-bounds

A. State variables extracted from consumption

\begin{tabular}{lcccc}
\hline$C C$ & 9 & $16 / 14$ & $14 / 14$ & $19 / 21$ \\
$M S V$ & 31 & $41 / 38$ & $41 / 42$ & $60 / 61$ \\
$B Y$ & $>100 />100$ & $>100 />100$ & $>100 />100$ \\
$P S T$ & 100 & $93 / 86$ & $112 / 106$ & $86 / 85$ \\
\hline B. State variables & 69 & & & \\
\hline$C C$ & 18 & $39 / 43$ & $33 / 46$ & $47 / 48$ \\
$M S V$ & 69 & $90 / 84$ & $>100 />100$ & $5 / 5$ \\
$B Y$ & 4 & $5 / 5$ & $5 / 5$ & $100 />100$ \\
\hline
\end{tabular}

The table reports the minimum values of the utility curvature parameter $\gamma$ at which the model-implied SDF satisfies the HJ (Column 1), Q (Column 2), M (Column 3), and $\Psi$ (Column 4) bounds using quarterly data for 1947:Q1-2009:Q4. Columns 2-4 has two entries in each cell that correspond to whether the filtered $\psi^{*}$ component of the SDF and, therefore, the filtered SDF are estimated using equation 6, reported on the left, or Equation 4, reported on the right. Panels A and B present results when the models' state variables are extracted from consumption data and asset market data, respectively. $C C, M S V, B Y$, and $P S T$ denote, respectively, the models of Campbell and Cochrane 1999, Menzly et al 2004, Bansal and Yaron 2004), and Piazzesi et al. 2007).

the unobservable state variables, $x_{t}$ and $\sigma_{t}^{2}$, in terms of the observables, $z_{m, t}$ and $r_{f, t}$. Following this approach, the pricing kernel in Equation A16 can be expressed as a function of observable variables:

$$
\ln M_{t}=c_{1}^{\prime}-\gamma \Delta c_{t}+c_{3}^{\prime}\left(r_{f, t}-\frac{1}{\kappa_{1}} r_{f, t-1}\right)+c_{4}^{\prime}\left(z_{m, t}-\frac{1}{\kappa_{1}} z_{m, t-1}\right),
$$

where the parameters $\left(c_{1}^{\prime}, c_{3}^{\prime}, c_{4}^{\prime}\right)$ are functions of the underlying time-series and preference parameters.

Since the model is calibrated at the monthly frequency, we obtain the pricing kernels at the quarterly and annual frequencies by aggregating the monthly kernels. For instance, the quarterly pricing kernel, $M^{q}$, is obtained as

$$
\ln M_{t}^{q}=-\gamma \Delta^{q} c_{t}+\ln \psi_{t}^{q},
$$

where $\Delta^{q} c_{t}$ denotes the quarterly $\log$-consumption difference and $\ln \psi_{t}^{q}$ is given by

$$
\ln \psi_{t}^{q}=3 c_{1}^{\prime}+\sum_{i=0}^{2}\left[c_{3}^{\prime}\left(r_{f, t-i}-\kappa_{1} r_{f, t-i-1}\right)+c_{4}^{\prime}\left(z_{m, t-i}-\kappa_{1} z_{m, t-i-1}\right)\right] .
$$

Therefore, using the monthly calibrated parameter values from Bansal and Yaron 2004 and the historical monthly time series of the market-wide price-dividend ratio and risk-free rate, we obtain the time series of the SDF and its missing component at the quarterly and annual frequencies from the above two equations.

\section{A.7 Additional Robustness Checks}

A.7.1 Entropy bounds when all model parameters are simultaneously allowed to vary. In the empirical analysis on the entropy bounds, we have focused on one-dimensional bounds as a function of the risk-aversion parameter, $\gamma$, while fixing the other parameters at the authors' calibrated values. In other words, we have computed the minimum values of $\gamma$ at which the modelimplied SDFs satisfy the $H J, Q, M$, and $\Psi$ bounds, while holding the remaining model parameters fixed at their calibrated values. As a robustness check, in this section, we compute the minimum values of $\gamma$ at which the model-implied SDFs satisfy the bounds, while allowing the remaining 
model parameters to simultaneously vary over two standard error intervals around their calibrated values.

For the external habit models of Campbell and Cochrane 1999) and Menzly et al. 2004, the model-implied SDFs are obtained by extracting the surplus consumption ratio from aggregate consumption data. While the state variable also may be extracted from the price-dividend ratio, the Menzly et al 2004 model admits a closed-form solution for the price-dividend ratio only for $\gamma=1$, and this motivates our choice for the extraction of the state variable in the external habit models. For the Bansal and Yaron 2004) long-run risks model, on the other hand, we extract the two state variables by inverting the closed-form solutions for the price-dividend ratio and riskfree rate. While the state variables also can be extracted from aggregate consumption data using Bayesian smoothing procedures, the computing time makes it prohibitively expensive to do this, while allowing all the parameters to vary simultaneously (since the Bayesian smoothing would have to be computed for each of set of parameter values considered). Finally, for the Piazzesi et al. 2007 model, the state variable is directly observable from the BEA tables and, therefore, does not need to be extracted from either consumption or asset market data.

The results are presented in Table A2. The table shows that, for each model, the $H J, Q, M$, and $\Psi$ bounds are satisfied for smaller values of $\gamma$ when the other parameters are allowed to vary simultaneously compared to Tables 6 and 7 where the other parameters are held fixed. However, as in the latter tables, the $C C, M S V$, and $P S T$ models still require much larger values of risk aversion to satisfy the bounds compared with the authors' calibrated values at the quarterly frequency.

A.7.2 Entropy bounds when the risk-free rate is included as an additional test asset. In the empirical analysis, we have used the excess returns (in excess of the risk-free rate) on a broad cross-section of risky assets to extract the SDF and obtain entropy bounds for the SDF and its components. As a robustness check, we repeat the empirical exercise using as test assets the gross returns (instead of excess returns) on the cross section of size- and book-to-market-equity-sorted, momentum-sorted, and industry-sorted portfolios, and the return on the risk-free asset.

In this case, the relevant Euler equation is

$$
\mathbf{1}_{N}=\mathbb{E}\left[m(\theta, t) \psi_{t} \mathbf{R}_{t}\right],
$$

where $\mathbf{R}_{t} \in \mathbb{R}^{N}$ is a vector of gross returns and $\mathbf{1}_{N}$ is an $N$-dimensional vector of ones. Under weak regularity conditions, the above pricing restrictions for the SDF can be rewritten as

$$
\bar{\psi}^{-1} \mathbf{1}_{N}=\mathbb{E}^{\Psi}\left[m(\theta, t) \mathbf{R}_{t}\right]
$$

or as

$$
\bar{M}^{-1} \mathbf{1}_{N}=\mathbb{E}^{Q}\left[\mathbf{R}_{t}\right],
$$

where $\bar{x} \equiv \mathbb{E}\left[x_{t}\right], \frac{\psi_{t}}{\psi}=\frac{d \Psi}{d P}$, and $\frac{M_{t}}{\bar{M}}=\frac{d Q}{d P}$. Therefore, Equations 44-7] can be reformulated, respectively, as Equations A21-A24 below:

$$
\hat{\Psi} \equiv \underset{\Psi}{\arg \min } D(\Psi \| P) \equiv \underset{\Psi}{\arg \min } \int \frac{d \Psi}{d P} \ln \frac{d \Psi}{d P} d P \text { s.t. } \bar{\psi}^{-1} \mathbf{1}_{N}=\mathbb{E}^{\Psi}\left[m(\theta, t) \mathbf{R}_{t}\right],
$$

with its dual solution given (up to a positive scale constant) by

$$
\hat{\psi}_{t}=\frac{e^{\lambda(\theta)^{\prime}\left[m(\theta, t) \mathbf{R}_{t}-\bar{\psi}^{-1} \mathbf{1}_{N}\right]}}{\left.\sum_{t=1}^{T} e^{\lambda(\theta)^{\prime}\left[m(\theta, t) \mathbf{R}_{t}-\bar{\psi}-1\right.} \mathbf{1}_{N}\right]}=\frac{e^{\lambda(\theta)^{\prime} m(\theta, t) \mathbf{R}_{t}}}{\sum_{t=1}^{T} e^{\lambda(\theta)^{\prime} m(\theta, t) \mathbf{R}_{t}}}, \forall t
$$

where $\lambda(\theta) \in \mathbb{R}^{N}$ is the solution to the following unconstrained convex problem

$$
\lambda(\theta) \equiv \underset{\lambda}{\arg \min _{\lambda}} \frac{1}{T} \sum_{t=1}^{T} e^{\lambda^{\prime}\left[m(\theta, t) \mathbf{R}_{t}-\bar{\psi}^{-1} \mathbf{1}_{N}\right]} ;
$$




$$
\hat{Q} \equiv \underset{Q}{\operatorname{argmin}} D(Q \| P) \equiv \underset{Q}{\operatorname{argmin}} \int \frac{d Q}{d P} \ln \frac{d Q}{d P} d P \text { s.t. } \bar{M}^{-1} \mathbf{1}_{N}=\mathbb{E}^{Q}\left[\mathbf{R}_{t}\right],
$$

with its dual solution given (up to a positive scale constant) by

$$
\hat{M}_{t}=\frac{e^{\lambda^{\prime} \mathbf{R}_{t}}}{\sum_{t=1}^{T} e^{\lambda^{\prime} \mathbf{R}_{t}}}, \forall t,
$$

where $\lambda \in \mathbb{R}^{N}$ is the solution to

$$
\begin{gathered}
\lambda(\theta) \equiv \underset{\lambda}{\operatorname{argmin}} \frac{1}{T} \sum_{t=1}^{T} e^{\lambda^{\prime}\left[\mathbf{R}_{t}-\bar{M}^{-1} \mathbf{1}_{N}\right]} \\
\hat{\Psi} \equiv \underset{\Psi}{\operatorname{argmin}} D(P \| \Psi) \equiv \underset{\Psi}{\arg \min } \int \ln \frac{d P}{d \Psi} d P \text { s.t. } \bar{\psi}^{-1} \mathbf{1}_{N}=\mathbb{E}^{\Psi}\left[m(\theta, t) \mathbf{R}_{t}\right],
\end{gathered}
$$

with its dual solution given (up to a positive scale constant) by

$$
\hat{\psi}_{t}=\frac{1}{T\left[1+\lambda(\theta)^{\prime}\left(m(\theta, t) \mathbf{R}_{t}-\bar{\psi}^{-1} \mathbf{1}_{N}\right)\right]}, \quad \forall t,
$$

where $\lambda(\theta) \in \mathbb{R}^{N}$ is the solution to

$$
\begin{gathered}
\lambda(\theta) \equiv \underset{\lambda}{\arg \min _{i}}-\sum_{t=1}^{T} \log \left(1+\lambda^{\prime}\left(m(\theta, t) \mathbf{R}_{t}-\bar{\psi}^{-1} \mathbf{1}_{N}\right)\right) ; \\
\hat{Q} \equiv \underset{Q}{\operatorname{argmin}} D(P \| Q) \equiv \underset{Q}{\arg \min } \int \ln \frac{d P}{d Q} d P \text { s.t. } \bar{M}^{-1} \mathbf{1}_{N}=\mathbb{E}^{Q}\left[\mathbf{R}_{t}\right]
\end{gathered}
$$

with its dual solution given (up to a positive scale constant) by

$$
\hat{M}_{t}=\frac{1}{T\left[1+\lambda(\theta)^{\prime}\left(\mathbf{R}_{t}-\bar{M}^{-1} \mathbf{1}_{N}\right)\right]}, \quad \forall t
$$

where $\lambda(\theta) \in \mathbb{R}^{N}$ is the solution to

$$
\lambda(\theta) \equiv \arg \min _{\lambda}-\sum_{t=1}^{T} \log \left(1+\lambda^{\prime}\left(\mathbf{R}_{t}-\bar{M}^{-1} \mathbf{1}_{N}\right)\right) .
$$

Two observations are in order about the above results. First, looking at the dual optimizations, it is clear that different $\bar{M}$ and $\bar{\psi}$ will now matter in determining the solution; that is, changes in the means will change the estimated SDF, and not simply as a scaling. Second, $\bar{M}$ can be calibrated easily, since from the Euler equation we have

$$
\bar{M} \equiv \mathbb{E}\left[m(\theta, t) \psi_{t}\right]=\mathbb{E}\left[1 / R_{t}^{f}\right],
$$

and, therefore, can be estimated using a sample analog. $\bar{\psi}$, on the other hand, can be recovered from

$$
\begin{gathered}
\bar{M} \equiv \mathbb{E}\left[m(\theta, t) \psi_{t}\right]=\operatorname{Cov}\left(m(\theta, t) ; \psi_{t}\right)+\bar{m} \bar{\psi}, \\
\therefore \bar{\psi}=\frac{\bar{M}-\operatorname{Cov}\left(m(\theta, t) ; \psi_{t}\right)}{\bar{m}} .
\end{gathered}
$$


Therefore, to calibrate $\bar{\psi}$, we can follow the following iterative procedure:

1. Set $\bar{\psi}=\frac{\bar{M}}{\bar{m}}=\frac{\frac{1}{T} \sum_{t=1}^{T} 1 / R_{t}^{f}}{\frac{1}{T} \sum_{t}^{T} m(\theta, t)}$ as a starting guess.

2. Given $\bar{\psi}$, use the above entropy minimization procedures to estimate $\left\{\hat{\psi}_{t}\right\}_{t=1}^{T}$ (up to a positive constant $\kappa$ ).

3. Identify the scaling constant $\kappa$ using the fact that, from the Euler equation for the risk-free rate, we have (as $T \rightarrow \infty)$

$$
\kappa \frac{1}{T} \sum_{t=1}^{T} m(\theta, t) \hat{\psi}_{t}=\frac{1}{T} \sum_{t=1}^{T} 1 / R_{t}^{f} \Rightarrow \kappa=\frac{\sum_{t=1}^{T} 1 / R_{t}^{f}}{\sum_{t=1}^{T} m(\theta, t) \hat{\psi}_{t}} .
$$

4. Compute an updated $\bar{\psi}$ using

$$
\bar{\psi}=\frac{\bar{M}-\kappa \widehat{\operatorname{Cov}}\left(m(\theta, t) ; \hat{\psi}_{t}\right)}{\bar{m}}=\frac{\frac{1}{T} \sum_{t=1}^{T} 1 / R_{t}^{f}-\kappa \widehat{\operatorname{Cov}}\left(m(\theta, t) ; \hat{\psi}_{t}\right)}{\frac{1}{T} \sum_{t}^{T} m(\theta, t),}
$$

where $\widehat{\operatorname{Cov}}($.$) is the sample analogue based covariance estimator.$

5. With the new $\bar{\psi}$ at hand, go back to Step 2 and repeat until convergence of $\bar{\psi}$ is achieved. Once converge is achieved, the exact estimate (no more up to a constant) of $\psi_{t}$ is given by $\kappa \times \hat{\psi}_{t}$.

Table A3 repeats the analysis in Table 6 when the set of assets consists of the gross returns (instead of excess returns) on the 6 size and book-to-market-equity-sorted portfolios of FamaFrench, 10 industry-sorted portfolios, 10 momentum-sorted portfolios, and the risk-free asset. The table shows that the inclusion of the risk-free rate as an additional asset in the estimation leaves the $H J, Q, M$, and $\Psi$ bounds on the SDF and its components virtually unchanged for all the asset pricing models considered.

The results in the other tables also remain largely similar on inclusion of the risk-free rate and are omitted for the sake of brevity.

\section{References}

Ait-Sahalia, Y. and A. W. Lo. 1998. Nonparametric estimation of state-price densities implicit in financial asset prices. Journal of Finance 53(2):499-547.

Ait-Sahalia, Y. and A. W. Lo. 2000. Nonparametric risk management and implied risk aversion. Journal of Econometrics 94(1-2):9-51.

Alvarez, F. and U. J. Jermann. 2005. Using asset prices to measure the persistence of the marginal utility of wealth. Econometrica 73(6):1977-2016.

Amemiya, T. 1985. Advanced econometrics. Harvard University Press, Cambridge: Mass.

Backus, D., M. Chernov, and S. E. Zin. 2011. Sources of entropy in representative agent models. Working Paper 17219, National Bureau of Economic Research.

Backus, D., M. Chernov, and S. E. Zin. 2014. Sources of entropy in representative agent models. Journal of Finance, 69(1):51-99.

Bakshi, G. S. and F. Chabi-Yo. 2012. Variance bounds on the permanent and transitory components of stochastic discount factors. Journal of Financial Economics, 105:191-208.

Bansal, R. and A. Yaron. 2004. Risks for the long run: A potential resolution of asset pricing puzzles. Journal of Finance 59(4):1481-509. 
Breeden, D. T. 1979. An intertemporal asset pricing model with stochastic consumption and investment opportunities. Journal of Financial Economics 7:265-96.

Breeden, D. T., M. R. Gibbons, and R. H. Litzenberger. 1989. Empirical test of the consumption-oriented CAPM. The Journal of Finance 44(2):231-62.

Brown, D. E. and R. L. Smith. 1990. A correspondence principle for relative entropy minimization. Naval Research Logistics 37(2).

Brunnermeier, M. K. and C. Julliard. 2007. Money illusion and housing frenzies. Review of Financial Studies 20(5).

Campbell, J. Y. 1996. Understanding risk and return. Journal of Political Economy 104(2):298-345.

Campbell, J. Y. and J. H. Cochrane. 1999. By force of habit: A consumption-based explanation of aggregate stock market behavior. Journal of Political Economy 107(2):205-51.

Cochrane, J. H. 1996. A cross-sectional test of an investment-based asset pricing model. Journal of Political Economy 104(3):572-621.

Constantinides, G. M. and D. Duffie. 1996. Asset pricing with heterogeneous consumers. Journal of Political Economy 104(2):219-40.

Constantinides, G. M. and A. Ghosh. 2011. Asset pricing tests with long run risks in consumption growth. The Review of Asset Pricing Studies 1:96-136.

Csiszar, I. 1975. I-divergence geometry of probability distributions and minimization problems. Annals of Probability 3:146-58.

Duffie, D. 2005. Dynamic Asset Pricing Theory. New Age International (P) Ltd.

Epstein, L. G. and S. E. Zin. 1989. Substitution, risk aversion, and the temporal behavior of consumption and asset returns: A theoretical framework. Econometrica 57:937-68.

Fama, E. F. and French, K. R. (1993). Common risk factors in the returns on stocks and bonds. The Journal of Financial Economics 33:3-56.

Hansen, L. 2014. Nobel lecture: Uncertainty outside and inside economic models. Journal of Political Economy 122(5):945-87.

Hansen, L. P., J. Heaton, J. Lee, and N. Roussanov. 2007. Intertemporal substitution and risk aversion. In J. Heckman and E. Leamer, editors, Handbook of Econometrics, volume 6 of Handbook of Econometrics, chapter 61. Elsevier.

Hansen, L. P., J. Heaton, and E. G. J. Luttmer. 1995. Econometric evaluation of asset pricing models. The Review of Financial Studies 8(2):237-74.

Hansen, L. P., J. C. Heaton, and N. Li. 2008. Consumption strikes back? measuring long-run risk. Journal of Political Economy 116(2):260-302.

Hansen, L. P. and R. Jagannathan. 1991. Implications of security market data for models of dynamic economies. Journal of Political Economy 99(2):225-62.

Hansen, L. P. and R. Jagannathan. 1997. Asssessing specification errors in stochastic discount factor models. The Journal of Finance 52:557-590.

Hansen, L. P. and T. J. Sargent. 2010. Fragile beliefs and the price of uncertainty. Quantitative Economics 1(1):129-162.

Hansen, L. P. and K. J. Singleton. 1983. Stochastic consumption, risk aversion, and the temporal behavior of asset returns. Journal of Political Economy 91:249-68.

Hayashi, F. 2000. Econometrics. Princeton University Press: Princeton.

Hodrick, R. J. and E. C. Prescott. 1997. Postwar u.s. business cycles: An empirical investigation. Journal of Money, Credit, and Banking 29(1):1-16. 
Jackwerth, J. C. and M. Rubinstein. 1996. Recovering probability distributions from option prices. Journal of Finance 51(5):1611-32.

Jaynes, E. T. 1957a. Information theory and statistical mechanics. The Physics Review 106(4):620-30.

Jaynes, E. T. 1957b. Information theory and statistical mechanics ii. The Physical Review 108(2):171-90.

Julliard, C. and A. Ghosh. 2012. Can rare events explain the equity premium puzzle? Review of Financial Studies 25(10):3037-76.

Kitamura, Y. (2006). Empirical likelihood methods in econometrics: Theory and practice. Cowles Foundation Discussion Papers 1569, Cowles Foundation, Yale University.

Kitamura, Y. and M. Stutzer. 2002. Connections between entropic and linear projections in asset pricing estimation. Journal of Econometrics 107(1-2):159-74.

Kreps, D. M. and E. L. Porteus. 1978. Temporal resolution of uncertainty and dynamic choice theory. Econometrica 46(1):185-200.

Lettau, M. and Ludvigson, S. (2001a). Consumption, aggregate wealth, and expected stock returns. Journal of Finance 56(3):815-49.

Lettau, M. and S. Ludvigson. 2001b. Resurrecting the (C)CAPM: A cross-sectional test when risk premia are time-varying. Journal of Political Economy 109:1238-86.

Lucas, Jr., R. E. 1978. Asset prices in an exchange economy. Econometrica 46:1426-14.

Lukacs, E. 1970. Characteristic Functions (2nd Edition). London: Hafner Publishing.

Lustig, H. N. and S. G. V. Nieuwerburgh. 2005. Housing collateral, consumption insurance, and risk premia: An empirical perspective. The Journal of Finance 60(3):1167-219.

Mankiw, N. G. and M. D. Shapiro. 1986. Risk and return: Consumption beta versus market beta. Review of Economics and Statistics 68:452-59.

Mehra, R. and E. C. Prescott. 1985. The equity premium: A puzzle. Journal of Monetary Economics 15(2):145-61.

Menzly, L., T. Santos, and P. Veronesi 2004. Understanding predictability. Journal of Political Economy, 112(1): $1-47$.

Mishkin, F. S. and E. N. White. 2002. U.s. stock market crashes and their aftermath: Implications for monetary policy. NBER Working Papers 8992, National Bureau of Economic Research, Inc.

Owen, A. B. 1988. Empirical likelihood ratio confidence intervals for a single functional. Biometrika 75(2): 237-49.

Owen, A. B. 1991. Empirical likelihood for linear models. The Annals of Statistics 19(4):1725-47.

Owen, A. B. 2001. Empirical Likelihood. Chapman and Hall.

Parker, J. A. and C. Julliard. 2005. Consumption risk and the cross-section of expected returns. Journal of Political Economy 113(1).

Piazzesi, M., M. Schneider, and S. Tuzel. 2007. Housing, consumption and asset pricing. Journal of Financial Economics 83(3):531-69.

Robinson, P. M. 1991. Consistent nonparametric entropy-based testing. Review of Economic Studies 58(3): 437-53.

Rosenberg, J. V. and R. F. Engle. 2002. Empirical pricing kernels. Journal of Financial Economics 64(3):341-72.

Ross, S. A. 2011. The recovery theorem. Working Paper 17323, National Bureau of Economic Research.

Rubinstein, M. 1976. The valuation of uncertain income streams and the pricing of options. Bell Journal of Economics 7:407-25.

Savov, A. 2011. Asset pricing with garbage. Journal of Finance 66(1):177-201. 
Schervish, M. J. 1995. Theory of statistics. Springer series in statistics. Springer-Verlag.

Stutzer, M. 1995. A bayesian approach to diagnosis of asset pricing models. Journal of Econometrics 68(2): 367-97.

Stutzer, M. 1996. A simple nonparametric approach to derivative security valuation. Journal of Finance 51(5):1633-52.

Ulrich, M. 2010. Observable long-run ambiguity and long-run risk. Columbia University Manuscript.

Weil, P. 1989. The equity premium puzzle and the risk-free rate puzzle. Journal of Monetary Economics 24:401-21.

White, H. 1982. Maximum likelihood of misspecified models. Econometrica 50:1-25. 